\title{
Efficient Table Lookup Algorithms for the Next Generation IP Networks
}

\author{
by \\ Zhen Xu

\begin{abstract}
A thesis submitted to
the Faculty of Graduate Studies and Research

in partial fulfillment of the requirements for the degree of
\end{abstract}

Doctor of Philosophy

School of Mathematics and Statistics

\author{
Carleton University \\ Ottawa, Ontario, Canada \\ January, 2005
}

(C) Copyright 2005

Zhen $\mathrm{Xu}$ 


$\begin{array}{ll}\begin{array}{l}\text { Library and } \\ \text { Archives Canada }\end{array} & \begin{array}{l}\text { Bibliothèque et } \\ \text { Archives Canada }\end{array} \\ \begin{array}{l}\text { Published Heritage } \\ \text { Branch }\end{array} & \begin{array}{l}\text { Direction du } \\ \text { Patrimoine de l'édition }\end{array} \\ \begin{array}{l}\text { 395 Wellington Street } \\ \text { Ottawa ON K1A ON4 }\end{array} & \begin{array}{l}\text { 395, rue Wellington } \\ \text { Ottawa ON K1A ON4 } \\ \text { Canada }\end{array}\end{array}$

Your file Votre référence

ISBN: 0-494-00808-3

Ourfile Notre référence

ISBN: 0-494-00808-3

NOTICE:

The author has granted a nonexclusive license allowing Library and Archives Canada to reproduce, publish, archive, preserve, conserve, communicate to the public by telecommunication or on the Internet, loan, distribute and sell theses worldwide, for commercial or noncommercial purposes, in microform, paper, electronic and/or any other formats.

The author retains copyright ownership and moral rights in this thesis. Neither the thesis nor substantial extracts from it may be printed or otherwise reproduced without the author's permission.
AVIS:

L'auteur a accordé une licence non exclusive permettant à la Bibliothèque et Archives Canada de reproduire, publier, archiver, sauvegarder, conserver, transmettre au public par télécommunication ou par l'Internet, prêter, distribuer et vendre des thèses partout dans le monde, à des fins commerciales ou autres, sur support microforme, papier, électronique et/ou autres formats.

L'auteur conserve la propriété du droit d'auteur et des droits moraux qui protège cette thèse. $\mathrm{Ni}$ la thèse ni des extraits substantiels de celle-ci ne doivent être imprimés ou autrement reproduits sans son autorisation.
In compliance with the Canadian

Privacy Act some supporting forms may have been removed from this thesis.

While these forms may be included in the document page count, their removal does not represent any loss of content from the thesis.
Conformément à la loi canadienne sur la protection de la vie privée, quelques formulaires secondaires ont été enlevés de cette thèse.

Bien que ces formulaires aient inclus dans la pagination, il n'y aura aucun contenu manquant.

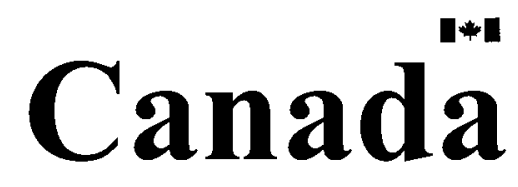




\begin{abstract}
The traffic on the Internet has exploded in recent years, due to a variety of new applications. Increasing the speed of packet transmissions and packet processing is a must. Optical fibers have already been used to make the transmission speed much faster, and there is still the potential to increase the bandwidth even wider in the near future.

Routers as packet processing devices have to keep up with the pace of transmission speed. Looking for the next hop of the packet (IP address lookup) and differentiating the traffic according to some criteria (packet classification) are among the bases in packet processing. The common feature of IP address lookup and packet classification is that both of them need to consult a table to determine the best matching rule. Table lookups for the next generation IP networks becomes a promising topic. This thesis is focused on addressing solutions to such problems. The goal for developing the algorithms is to optimize the search strategy to meet the requirements of the next generation IP networks. In that, the key issues are efficient memory access time and storage consumption, easy implementation, and scalability to different tables and IPv6. In this thesis, one IP address lookup algorithm, one packet classification algorithm, and efficient TCAM applications are proposed.
\end{abstract}

An IP address lookup algorithm based on Comb Extraction Scheme is proposed in Chapter 4 . The target is to transform a sequential $\mathbb{P}$ address process to a pair of smaller balanced sub-lookup processes and a comparison process, which are able to perform in parallel. The experimental results show that it has outstanding performance in both average search speed and storage cost.

A packet classification algorithm using bit characteristics is proposed in Chapter 5 . The target is to optimize the search path for large classifiers. This algorithm consists of iii 
three stages, hash table lookup, optimal search trie lookup, and small set lookup. The search path decision needs to take into account the bits chosen from all the dimensions at each step. It can overcome the problem of an unbalanced distribution of rules among dimensions. The experimental results provide strong support for the algorithm design.

Techniques that allow TCAMs more efficient in table lookups are proposed in Chapter 6. Three techniques are proposed to reduce TCAMs power consumption, increase the capability to handle bigger tables, and solve range matching problems in TCAMs, respectively. The experimental results verify that they have excellent performances.

The prominent feature of our algorithms is that they use novel optimal lookup methods, avoiding the traditional search order. They are amenable to parallel implementations. The experimental results show that they are not only comparable to current solutions but also have better performance for the table lookup at the edge.

We describe the background in this field and review some existing classical technologies of table lookups in the first three chapters. After that, the proposed algorithms are detailed in each of the following three chapters. Finally, we conclude in the last chapter. 


\section{Acknowledgements}

First of all, I would like to thank my supervisors Dr. Yiqiang Q. Zhao and Dr. Ioannis Lambadaris for their patient guidance, valuable suggestions and enlightening comments during the period of my study at Carleton University. Their profound knowledge has definitely benefited me a lot. Working with them has always been an enjoyable and rewarding experience.

Secondly, I really appreciate Dr. Gerard Damm and his colleagues at Alcatel Canada for providing invaluable suggestions and comments on my research work. I would like to thank Dr. Xuehong Sun, Xiaojun Nie, and Jun Li for providing some discussions and assistance in my studies. I also would like to thank Ryan Mackinnon who spent a lot of time proofreading a draft of this thesis.

Thirdly, I would like to thank the School of Mathematics and Statistics, Carleton University, for providing necessary research facilities for preparing this thesis. Many thanks go to members and staff of the School of Mathematics and Statistics, who helped me during my studies at Carleton University.

Finally, I would like to thank my husband, Jun Sun. I could not have completed this thesis without his love and support. I would like to thank my parents. I learned a lot from their positive attitude towards life under any circumstances. I would also like to thank my dear friend Lei Zhang, who invigorated me when I was under pressure. This thesis is a memorial to my beloved brother who passed away after a heroic battle against lymph cancer in February, 2004. Wish him the best in the heaven. 


\section{Contents}

Title Page $\quad$ i

Acceptance Sheet ii

Abstract iii

Acknowledgements $\quad$ v

$\begin{array}{lll}\text { Contents } & \text { vi }\end{array}$

1 Introduction 1

2 Background 5

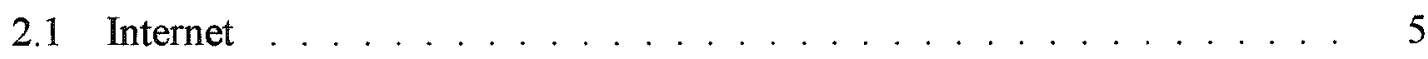

2.2 Quality of Service $(\mathrm{QoS}) \ldots \ldots \ldots \ldots$

2.3 Network Processors Based Routers . . . . . . . . . . . . . . . . . . . . . 7

2.4 Network Processors (NPs) $\ldots \ldots \ldots \ldots$

2.5 Address Architecture . . . . . . . . . . . . . . . . . . . . 12

2.5.1 IPv4 Address Architecture . . . . . . . . . . . . . . . . . 12

vi 
2.5.2 IPv6 Address Architecture . . . . . . . . . . . . . . . . 13

2.6 Matching Strategies . . . . . . . . . . . . . . . . . . . . . . . . . . 14

2.7 One-Dimensional Table Lookup: IP Address Lookup . . . . . . . . . . . 15

2.7.1 Forwarding Table . . . . . . . . . . . . . . 16

2.7.2 Difficulty of Internet Address Lookup . . . . . . . . . . . . . . . 17

2.8 Multi - Dimensional Table Lookup: Packet Classification . . . . . . . . . 18

2.9 Goals and Metrics for Table Lookups Algorithms . . . . . . . . . . . . 21

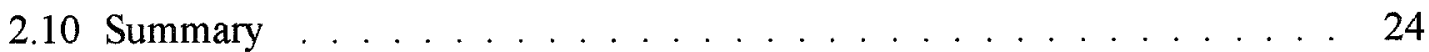

3 Related Table Lookup Algorithms 25

3.1 One-dimensional Table Lookup Algorithms - IP Address Lookup Algo-

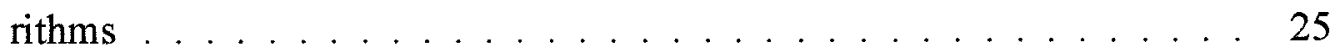

3.1 .1 Simple Schemes . . . . . . . . . . . . . 26

3.1 .2 Advanced Algorithms . . . . . . . . . . . . . . . . . . 29

3.2 Multi-Dimensional Table Lookup Algorithms -Packet Classification . . 37

3.2.1 Geometric Representation . . . . . . . . . . . . . . . . 38

3.2 .2 Trie Based Algorithms . . . . . . . . . . . . . . . . . 39

3.2.3 Comprehensive Search Algorithms . . . . . . . . . . . . . . . 44

3.3 Summary . . . . . . . . . . . . . . . . . . . 49

4 IP Address Lookup Based on CES

4.1 Introduction . . . . . . . . . . . . . . . 50

4.2 New Data Structure . . . . . . . . . . . . . . . . . 53

4.2.1 Comb Extraction Scheme (CES) . . . . . . . . . . . . . . . 53

4.2.2 The Decomposition of a Forwarding Table . . . . . . . . . . . . 54

vii 
4.3 Comparison Set ..................... 63

4.4 Performance Description . . . . . . . . . . . . . . . . 65

4.4.1 CES + Index tables . . . . . . . . . . . . . . 66

4.4 .2 CES + Binary Tries . . . . . . . . . . . . . 70

4.4.3 Possible Variant Discussion . . . . . . . . . . . . . 71

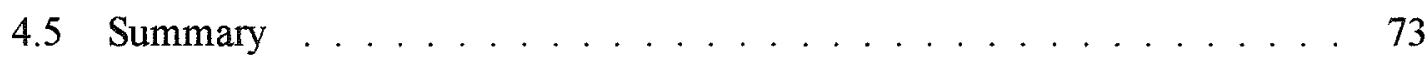

5 Packet Classification Using Bits Characteristics $\quad 74$

5.1 Introduction . . . . . . . . . . . . . . . . . . . 74

5.2 The Structure of the Proposed Algorithm . . . . . . . . . . . . 76

5.2 .1 Hash Table . . . . . . . . . . . . . . . . 77

5.2 .2 Search Trie . . . . . . . . . . . . . . . 86

5.2 .3 Small Set . . . . . . . . . . . . . . . 92

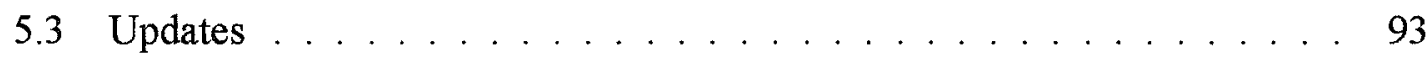

5.4 An Example . . . . . . . . . . . . . . . . . . . . . . . . . 94

5.5 Performance Analysis . . . . . . . . . . . . . . . . . 96

5.5.1 Performance for 2-Dimensional Packet Classification . . . . . . . 96

5.5.2 Performance for 5-Dimensional Packet Classification . . . . . . 105

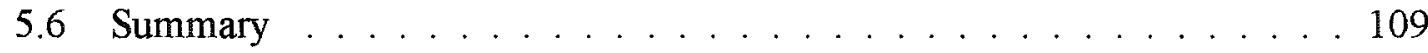

6 Applications of Efficient TCAMs in Table Lookups 111

6.1 Introduction . . . . . . . . . . . . . . . 111

6.2 Reducing TCAMs Power Consumption - Segment Enablement . . . . . 113

6.2.1 Elementary Bit Selection . . . . . . . . . . . 115

6.2 Precise Bit Selection . . . . . . . . . . . . . 116

viii 
6.3 Increasing the Table Size TCAMs Can Handle - Entries Compaction . . 119

6.4 Range Matching in TCAMs . . . . . . . . . . . . . . . . . . 123

6.4.1 Range Encoding Scheme (RES) . . . . . . . . . . . . . 124

6.4.2 RES + TCAM in Packet Classification . . . . . . . . . . . . . . 130

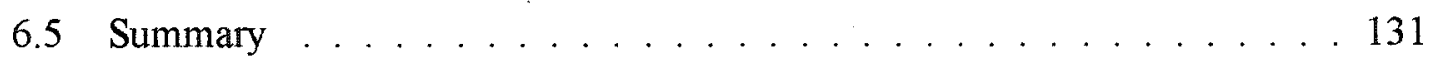

7 Conclusions and Future Work 132

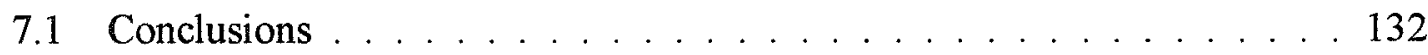

7.1.1 Contributions of the IP Address Lookup Based on Comb Extraction Scheme . . . . . . . . . . . . . . . . 133

7.1.2 Contributions of the Packet Classification Using Bit Characteristics 133

7.1.3 Contributions of the TCAMs in Table Lookup Algorithms . . . . 134

7.2 Future Work . . . . . . . . . . . . . . . . . 135

$\begin{array}{lr}\text { Bibliography } & 137\end{array}$

ix 


\section{List of Figures}

2.1 A Router Architecture . . . . . . . . . . . . . . . . . . . . . . . . 9

2.2 General Architecture of a Network Processor . . . . . . . . . . . . 11

2.3 Growth of a Forwarding Table . . . . . . . . . . . . . 17

3.1 Address Lookup in a TCAM . . . . . . . . . . . . . . 28

3.2 A Binary Trie . . . . . . . . . . . . . . . 30

3.3 A Path Compressed Trie . . . . . . . . . . . . . . . . 32

3.4 An Multibit Trie . . . . . . . . . . . . . . . . . . . . . 33

3.5 A Binary Search Tree . . . . . . . . . . . . . . . . . . . 35

3.6 A Simple Hash Function $\ldots \ldots \ldots$. . . . . . . . . . . 36

3.7 Hash Tables for Each Possible Prefix Length . . . . . . . . . . . . 37

3.8 Binary Search Tree and Hash Table . . . . . . . . . . . . . 38

3.9 Geometric Representation of the Two-Dimensional Classifier of Table $3.3 \quad 40$

3.10 Basic Trie of Tries . . . . . . . . . . . . . . . . 41

3.11 Grid of Tries With the Use of Switch Pointers . . . . . . . . . . . 42

3.12 Two Tries Associated With Each of the Fields In Table 3.3, together with both the bit vectors and the aggregate bit vector. The aggregate bit vector has 3 bits using an aggregation size of 4 .

$\mathrm{X}$ 
3.13 Decomposing the Space Recursively. . . . . . . . . . . . . . . . . . . 44

3.14 Decomposing the Space Recursively. . . . . . . . . . . . . . . . . . . 45

3.15 The Mapping of $S$ Bits to $T$ Bits . . . . . . . . . . . . . . . . . . . . 46

3.16 The Packet Flow in RFC . . . . . . . . . . . . . . . . . 47

3.17 The Structure of HiCuts for Table $3.3 \ldots \ldots$. . . . . . . . . . . . . . 48

4.1 Examples of Using CES . . . . . . . . . . . . . . 53

4.2 Architecture of the Algorithm f . . . . . . . . . . . . . . 67

4.3 Structure of an Entry in the Sub Index Table . . . . . . . . . . . . . 68

4.4 The Structure of CES + Index tables . . . . . . . . . . . . . . 69

5.1 The Structure of the Proposed Algorithm . . . . . . . . . . . . . . 76

5.2 Hash results . . . . . . . . . . . . . . . . . . . . . . 79

5.3 The Expansion for 2-Dimensional Entries . . . . . . . . . . . . . . 82

5.4 Hash Lookup Flow Chart . . . . . . . . . . . . . . . . . . . . . . 85

5.5 Comparison of Different Bit Selection Methods (1) . . . . . . . . . . . 87

5.6 Comparison of Different Bit Selection Methods (2) . . . . . . . . . . . 90

5.7 An Example for 2-Dimensional Packet Classification . . . . . . . . . . . 95

5.8 Length of the Hash Table . . . . . . . . . . . . . . . . . . . . . . . . . . . 98

5.9 Mean Collisions in a Hash Bucket . . . . . . . . . . . . . . . . . . . . . 99

5.10 Maximum Collisions in a Hash Bucket . . . . . . . . . . . . . . . . . . . 100

5.11 Depth of the Search Trie . . . . . . . . . . . . . 101

5.12 Storage Cost Comparison Between Using Different Hash Functions . . . 102

5.13 Comparison of the Storage Cost When MAXBITS Varies . . . . . . . . 103

5.14 Comparison of the Search Steps When MAXBITS Varies . . . . . . . . 104

$$
\text { xi }
$$


6.1 Proposed Architecture for TCAM-Based IP Address Lookup . . . . . . . 114

6.2 Compacting Entries in a Forwarding Table Using Mask - Based Aggregation 120

6.3 Compacting Entries in a Forwarding Table Using Overlapping Aggregation 121

6.4 Re-Encode Filters According to Different Levels . . . . . . . . . . . . 126

6.5 Extended TCAM-Based Packet Classification . . . . . . . . . . . . 129

xii 


\section{List of Tables}

2.1 A Small Sample Forwarding Table . . . . . . . . . . . . . . 16

2.2 The Service and Its Relevant Packet Fields in a Classifier . . . . . . . . 19

2.3 An Example of a Classifier in Four Dimensions _ . . . . . . . . . 20

2.4 Lookup Speed Table . . . . . . . . . . . . . . . . . . . . . . . 23

3.1 A Simple One-Dimensional Forwarding Table . . . . . . . . . . . . . 26

3.2 Memory Technologies . . . . . . . . . . . . . . . . . . . . . . . . . 29

3.3 A Sample of Two-Dimensional Classifier . . . . . . . . . . . . . . 39

4.1 A Sample Forwarding Table . . . . . . . . . . . . . . . . 52

4.2 Sub-Forwarding Table 1 from Table 4.1 (extracting bits in odd positions) . 55

4.3 Sub-Forwarding Table 2 from Table 4.1 (extracting bits in even positions) 56

4.4 Performance of the Pair Sub-tables by Using CES . . . . . . . . . . 62

4.5 Performance of the Pair Sub-tables by Such a Splitting Rule: extracting the higher 16 bits to form sub-table 1 and extracting the lower 16 bits to form sub-table $2 \ldots \ldots \ldots \ldots \ldots \ldots$

4.6 Delay for the Comparison between One Pair of Sub-Prefixes . . . . . 65

4.7 Storage Cost Comparison (CES+Binary Trie VS Binary Trie) (in Byte) . . 70

xiii 
5.1 A Small Two-Dimensional Classifier . . . . . . . . . . . . . . . . 94

5.2 Hash Table Size . . . . . . . . . . . . . . . . . . 106

5.3 Subentries Distribution Using SUMs Hash Function (\# of Rules = 256K) 107

5.4 Algorithm Performance (\# of Rules $=128 \mathrm{~K}, M A X B I T S=4, L E A F S I Z E=$

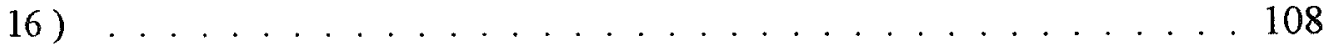

6.1 Results of Using the Elementary Bit Selection . . . . . . . . . . . 115

6.2 Result of Selecting the First 8 bits to Split the Forwarding Table . . . . . 116

6.3 Result of Combining the Precise Bit Selection . . . . . . . . . . . . . 118

6.4 Comparisons Between Table 6.3 and Table 6.1 . . . . . . . . . . . . . . . 119

6.5 Results After Entries Comparisons . . . . . . . . . . . . . . . . . . . . . 122

6.6 An Example of Range Set . . . . . . . . . . . . . . . . . . . 125

6.7 Width of Encoding Sequences . . . . . . . . . . . . . 130 


\section{List of Acronyms}

ABV Aggregate Bit Vector

ASIC Application Specific Integrated Circuit

BGP Border Gateway Protocol

BMP Best Matching Prefix

CES Comb Extraction Scheme

CIDR Classless Inter-Domain Routing

DiffServ Differentiated Service

DWDM Dense Wavelength Division Multiplexing

GPP General Purpose Processor

HDTV High Definition TV

HiCut Hierarchical Intelligent Cuttings

IntServ Integrated Service

IP Internet Protocol

ISP Internet Service Providers

MC Management Controller

MPLS Multi-Protocol Label Switching 
NAP Network Access Point

NPU Network Processor Unit

QoS Quality of Service

RC Router Controller

RES Range Encoding Scheme

RISC Reduced Instruction Set Computer

SLA Service Level Agreement

SoC System on Chip

SONET Synchronous Optical NETwork

SRAM Static Random Access Memory

DRAM Dynamic Random Access Memory

TCAM Ternary Content Addressable Memory

TCP Transmission Control Protocol

ToS Type of Service

VLSI Very Large Scale Integration

VoIP Voice over IP

VPN Virtual Private Network

$\mathrm{xvi}$ 


\section{Chapter 1}

\section{Introduction}

The traffic in the Internet grows drastically in recent years. To sustain growth, Internet Service Providers (ISPs) provide different levels of service assurances to the various forms of traffic. It allows the applications to be timing and Quality of Service (QoS) guaranteed to Differentiated Services (DiffServ) and Integrated Services (IntServ) [58]. A router is not a simple network device to establish routing paths for packets any more. It is also able to distinguish packets belonging to different flows. IP Address Lookup and Packet Classification are two main steps for packet processing in Internet routers.

In an IP Address Lookup operation, the packet's destination address is used as key to consult the forwarding table to make forwarding decision. It needs to find the longest matching prefix and the corresponding next-hop router the packet should be sent [42]. In a Packet Classification operation, the packet needs to be classified by trying to find the most specific match with the highest priority from a large number of rules in a classifier, and then the corresponding action is performed or the required service level is established. 
The classifier can be any combination of network address, protocols and ports [54]. In fact, IP address lookup can be regarded as a special case of packet classification, in that IP address lookup is a one-dimensional table lookup problem while packet classification is a multi-dimensional table lookup problem.

The invention and evolution of Dense Wavelength Division Multiplexing (DWDM) technology has brought a breakthrough to high-speed networks. It provides $10 \mathrm{Gbps}$ data rate for today's network, and there is still potential to make the bandwidth much wider in the near future (40Gbps data rate). The rate of packet processing in Internet routers have to keep pace with the continuously increasing wire rate speed, even when multiple parallel applications are performing in the Network Processor Unit (NPU) [7]. IPv6 [86], however, will definitely replace IPv4, which significantly degrades the performance of the existing fast lookup approaches. High performance IP address lookup and packet classification algorithms have been a promising topic today for the next generation IP networks.

The key challenges in designing and developing IP address lookup and packet classification algorithms are to reduce memory space requirement, to increase processing speed, and to be applied for large and high-dimensional databases of classification rules $[14,18,42]$.

The current market of IP lookup and packet classification is dominated by TCAM solutions. Many companies, such as IDT [82], Cypress [84], NetLogic Microsystems [83], and SiberCore Technologies [81], have the search engine products use TCAMs for table lookup. However, the limitation of TCAMs, low density and high cost, impede their utilizations for large table lookups. Therefore, SRAM-based, DRAM-based, and ASICplus-SRAM-based solutions are the new directions for IP address lookup and packet clas- 
sification. Several companies have adopted algorithm-based solutions, such as Ezchip Technologies [77] and Hywire [78]. Also, it is regarded as one of the most important research areas in Alcatel and Nortel. Under this background, the thesis was developed. Our goals are to develop algorithms to address the current market needs. The highlights of the thesis are in three fold:

- Parallelize one-dimensional sequential lookup process;

- Optimize the search path with respect to different bit distributions;

- Improve the performance of TCAMs.

In this thesis, an IP address lookup algorithm, a packet classification algorithm and some novel techniques to allow TCAMs in table lookups more efficient are proposed. The features of these approaches are: small memory requirement, fast search speed, and scalable to IPv6. We summarize the features of these algorithms as follows:

1. One parallel IP address lookup algorithm will be proposed in this thesis. General trie based or range based IP address lookup algorithms are sequential processes. They waste time on traversing the branches. On the other hand, index table lookup can achieve the lower bound of search time, but it is not practical due to its huge memory demand. A novel method, called Comb Extraction Scheme (CES), will be proposed to decompose an original lookup system into a pair of parallel sub-lookup systems and one comparison system. Therefore, a single sequential process is able to be transformed into multiple parallel processes. Distinct architectures and the corresponding search strategies will be presented. The experiments show that CES based architecture makes a big improvement on traditional search algorithms, both in time complexity and in storage complexity [64]. 
2. One high-level search algorithm for packet classification will be presented in this thesis. It is composed of three layers: hash table lookup, search trie lookup and linear (or parallel) search in a small set. A novel hash function is proposed, which is built based on the bits from all the fields. It is able to reduce the maximum collisions in one hash bucket. An optimal multi-way search trie is built in each bucket. It only needs to inspect some most significant bits chosen from all the fields, instead of contiguous bits. A final small set can be achieved in limited steps. It has better performance in time complexity and storage complexity for large unbalanced classifiers over other existing algorithms [65].

3. Extended TCAM applications in IP address lookup algorithm and packet classification algorithm will also be presented in this thesis. TCAM is a compact unit for multiple parallel comparing processing. However its low density volume, high power consumption, and poor range matching ability have always been the drawbacks. Those limitations have prevented it from being deployed in today's market. In this thesis, solutions to address these problems in table lookups will be presented, in order to improve TCAM's performance in table lookups [66].

The thesis is organized as follows. The background knowledge in this field is given in Chapter 2. In Chapter 3, some classical technologies of IP address lookup and packet classification will be reviewed. One new IP address lookup algorithm and one novel packet classification algorithm are proposed in Chapter 4 and Chapter 5, respectively. Some techniques using efficient TCAMs are presented in Chapter 6. Conclusions are given in Chapter 7. 


\section{Chapter 2}

\section{Background}

In this chapter, the background of IP address lookup and packet classification is given firstly. Then the challenges of the IP address lookup and packet classification algorithms are described. The purpose of this chapter is to provide a brief introduction to this field.

\subsection{Internet}

The transformation of the Internet into an important and ubiquitous commercial infrastructure has not only created rapidly rising bandwidth demand but also significantly changed consumer expectations in terms of performance, security, and services. Consequently, ISPs need to not only evolve their networks to higher and higher speeds but also need to address the varied requirements of increasingly sophisticated services of different customers. ISPs would also like to maximize the sharing of the costly backbone 
infrastructure in a manner that enables them to control usage of network resources in accordance with service pricing and revenue potential. The two trends of rapidly rising bandwidth demand and the rising need for differentiation have resulted in intense efforts to build fast packet forwarding engines and to define mechanisms for service differentiation $[52,53,60,76]$.

Individual computers are connected to form a subnet. The subnets are connected to the Internet core through the edge routers. In order to provide good QoS, four key factors are needed to deal with the increasing traffic demand: higher link speed, better router data throughput, faster packet processing rate, and quick adaptation to routing changes [19]. Nowadays optical fibers are used in real networks. It can transmit data in tens of gigabit per second per fiber. High rates like OC48(2.5Gbit/s) and OC192(10Gbit/s) are common in the current market. OC768(40Gbit/s) has been achieved and will be used in the near future. It is easy to imagine that the bottleneck on end-to-end communications is in routers. So high performance routers are needed to keep pace with the increasing wire-speed [47].

\subsection{Quality of Service (QoS)}

QoS means providing a consistent, predictable data delivery service. It is able to give priority to certain mission critical applications in the network, maximize the use of the current network investment in infrastructure, provide better performance for delay sensitive applications such as Voice and Video, and respond to changes in network traffic flows [54]. It is the ability of a network element to have some level of assurance that its traffic and service requirements can be satisfied. To enable QoS requires the cooperation 
of all network layers from top to bottom, as well as every network element from end to end. Service availability, throughput, delay, jitter, and packet loss ratio are regarded as five most important QoS performance criteria of [38].

Integrated Service (IntServ) and Differentiated Service (DiffServ) are two different approaches to QoS on IP network. IntServ requires policy control over the creation of signaled reservations, which provide specific quantitative end-to-end behavior for a (set of) flows. It aims at providing real-time services and an ability to share dynamically the available link capacity in a controlled manner. DiffServ requires policy to define the correspondence between codepoints in the packet's DS-field and individual per-hop behaviors. It aims at providing differential treatments to flows or aggregates of flows by using the Type of Service bye in the IP header $[15,38]$.

The technologies to support QoS include classification, policing, scheduling, congestion control and shaping. Classification itself performs first to allow the corresponding strategies to be utilized. Classification is the task of identifying traffic coming into the network and setting its priority based on a number of factors, including the application, the policies of the service provider, and the services requested by the subscriber. Classification in DiffServ usually is typically done at the edges of a network, leaving the backbone or core to do more in the way of scheduling and efficient routing [15]. Packet scheduling and packet training are applied, so that no high-priority traffic is delayed.

\subsection{Network Processors Based Routers}

Generally, routers have experienced four generations [21]. The first generation routers 
used a single General Purpose Processor (GPP) and its internal data path was a bus. The line cards and GPP communicated with each other through a shared bus. Obviously, the performance of a router heavily depends on the throughput of the shared bus and forwarding speed of the GPP, which are the bottlenecks.

The second generation routers used distributed GPPs instead of the centralized one. Each line card had its own processor. However the shared bus cannot scale to higher capacity links.

In the third generation routers, the congested shared bus is replaced by a crossbar switch providing sufficient bandwidth for transmitting packets between line cards and allowing throughput to be increased by several orders of magnitude. The new bottleneck becomes packet processing instead of the forwarding path between line cards.

GPPs can no longer handle large link capacities. Value added services such as QoS and Virtual Private Network (VPN) are performed in routers and they require detailed classification and processing for each packet. GPPs cannot keep up with these increasing demands. Hence, Network processors (NPs) based routers are developed as the next generation of high-performance routers.

Figure 2.1 illustrates a typical router structure, where multiple line cards, a router controller (RC) and a management controller (MC) are interconnected with a switch fabric. The tasks such as participating in routing protocols, reserving resources, handling packets that need extra attention, and other administrative and maintenance operations, are handled by the RC and the MC. The line cards are the entry and exit points of data to and from a router. They provide the interface from physical and higher layers to the switch fabric. The tasks provided by line cards are becoming more complex as new applications develop and protocols evolve [7]. 


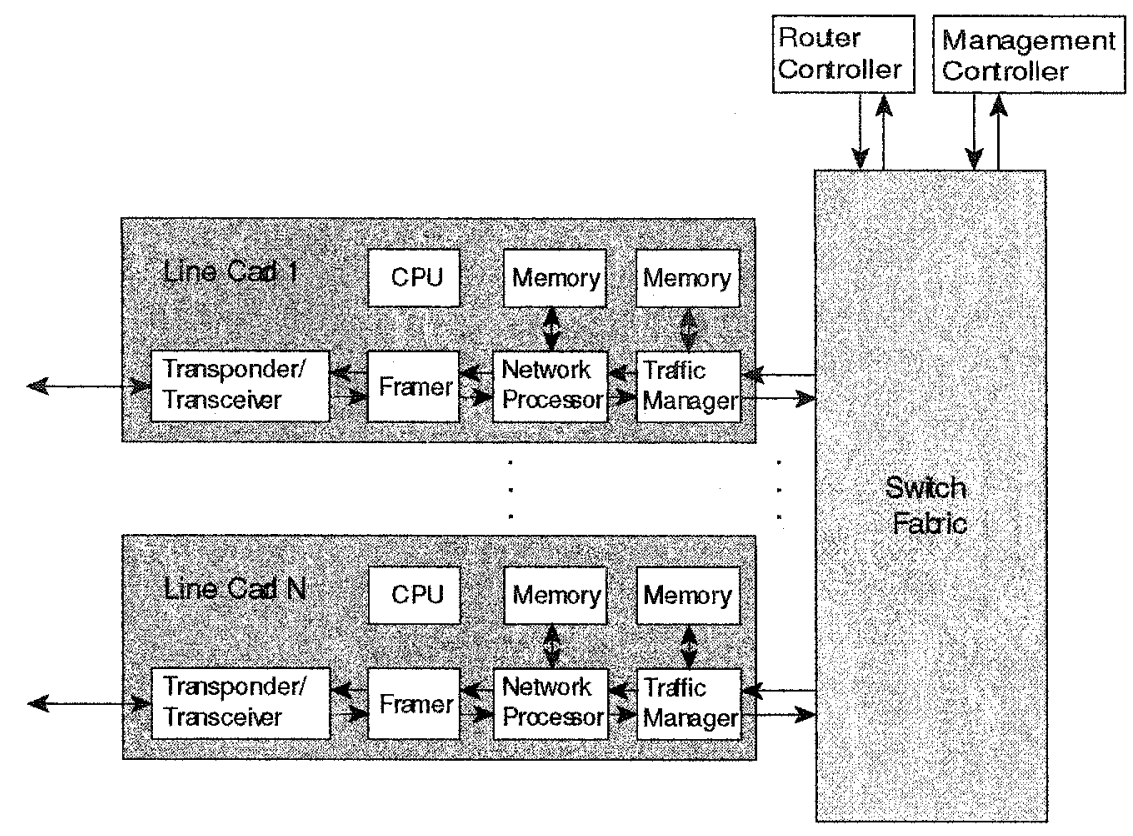

Figure 2.1: A Router Architecture

Each line card supports at least one full-duplex fiber connection on the network side, and at least one ingress and one egress connection to the switch fabric backplane. Generally speaking, for high-bandwidth applications, the network connections support channelization for the aggregation of lower-speed lines into a large pipe, and the switch fabric connections provide flow-control mechanisms for several thousand input and output queues to regulate the ingress and egress traffic to and from the switch fabric.

A line card usually includes components such as a transponder, framer, network processor (NP), traffic manager, and $\mathrm{CPU}$.

Transponder/Transceiver performs optical-to-electrical and electrical-to-optical signal conversions and serial-to-parallel and parallel-to-serial conversions.

Framer performs synchronization, frame overhead processing, and cell or packet delin- 
eation.

NP It mainly performs IP address lookup, packet classification, and packet modification.

Traffic Manager performs various control functions to cell/packet streams, including traffic access control, buffer management, and cell/packet scheduling, to meet each connection's and service class' requirements.

CPU performs control plane functions including connection setup/tear-down, table updates, register/buffer management, and exception handling.

\subsection{Network Processors (NPs)}

NPs [72] have received serious considerations over GPPs for the design of network equipment. It is a fundamental part of routers and other network equipments. As voice and data network applications continue their ascent toward convergence, not only is the combination of speed and flexibility becoming increasingly important, but also the processing of multiple services from Layer 2 through Layer 7 is becoming more crucial. Requirements for $\mathrm{QOS}$ and efficiently managed multi-vendor networks are driving a wealth of new capabilities, such as Multi-Protocol Label Switching (MPLS), voice-over-data services, support for IPv6, and service level agreement (SLA) accounting and control. At the same time, the range of different interfaces, from $\mathrm{T} 1$ through $\mathrm{OC}-192$, is increasing rather than decreasing. The ability to support emerging sophisticated applications is becoming increasingly demanding in today's high-capacity networks [7].

An NP is typically made up of a main controller, multiple task units and a central 


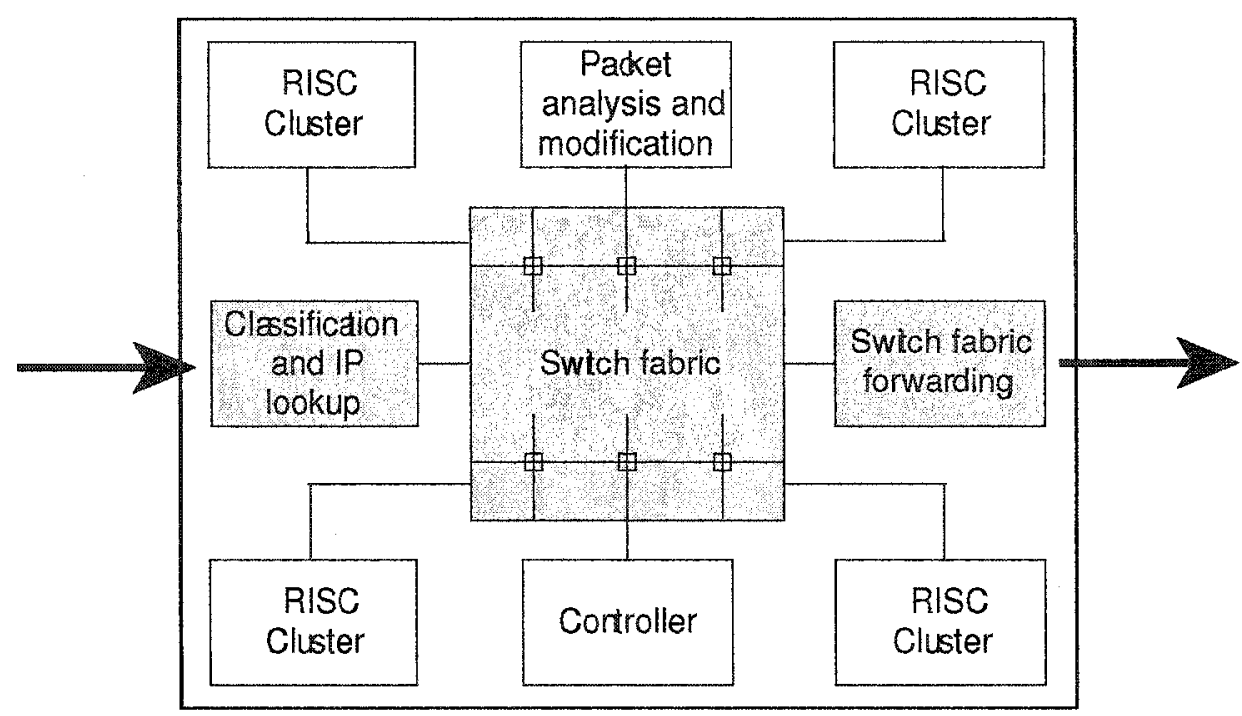

Figure 2.2: General Architecture of a Network Processor

switch fabric. A task unit can be a cluster of RISC processors or a special-purpose coprocessor. The controller handles the downloading of the instruction set to each RISC processor, the access of a RISC processor to special-purpose coprocessors and memory, and the configuration of the switch fabric.

Figure 2.2 depicts a simple general architecture of an NP. When a packet arrives at the NP, the controller assigns an idle RISC processor to handle the processing of the packet. The RISC processor may perform the classification function by itself or forward the packet to the classification coprocessor. The latter approach allows a new function to be performed by the RISC processor and a specific function to be performed by a coprocessor. After the packet is classified, the RISC processor may perform the packet modification or forward the packet to a modification coprocessor. The processing of the packet continues until it is done. Then the task unit informs the controller, which schedules the departure time for the processed packet. 
IP address lookup or packet classification is often the first step in packet processing in a network processor. It is required that operation is at the line speed, even when multiple parallel applications are performing in the Network Processor. It is essential to find a better solution to IP lookup and packet classification to meet the demand of the NPs in a real network systems.

\subsection{Address Architecture}

In this section, we will discuss the IPv4 and IPv6 address architectures, in order to understand IP address lookup and packet classification algorithms well.

\subsubsection{IPv4 Address Architecture}

An IPv4 address is a 32-bit binary address represented as dotted decimal of the form M. N. O. P, where $M, N, O$, and $P$ are decimal values ranging from $0-255$. An address initially uses a simple two-level hierarchy, with a network ID at the top level and a host at the bottom level. The network ID identifies the network to which a host is attached, and thus all hosts attached to the same network agree in the network ID of their IP addresses. An address's network ID is called a prefix. Depending on the segmentation, the addresses are grouped into Class A, Class B, and Class C addresses. Class D and Class E are reserved address spaces. In Class $A$, the first 8 bits, beginning with 0 , are defined as the network ID and the following 24 bits are defined as the host ID, which is represented by an 8-24 division structure. Class B, beginning with 10, has a 16-16 structure, and Class $\mathrm{C}$, beginning with 110 , has a $24-8$ structure. The number of bits assigned to a network field 
in class-based IP addresses can only be predefined as 8,16 , and 24 , respectively. This scheme is neither efficient nor flexible in using the IP addresses [85].

The massive growth of the Internet results in the rapid growth of the size of the forwarding table in a router and the depletion of the address space in IPv4. These problems have been migrated by the adoption of Classless Inter Domain Routing (CIDR). Any number of bits can be assigned to the network field. In CIDR notation, an IP address is represented as A.B.C.D $/ \mathrm{n}$, where " $/ \mathrm{n}$ " is called the IP prefix or network prefix. The IP prefix identifies the number of significant bits used to identify a network. For example, 192.9.205.22/18 means, the first 18 bits are used to represent the network and the remaining 14 bits are used to identify hosts. Using CIDR IP addressing, any number of contiguous bits can be assigned to identify networks, depending on the number of hosts it needs to support. This will greatly reduce the number of wasted IP addresses. In CIDR, a single entry is used to represent a group of class-based networks. Therefore, CIDR also reduces the number of entries in the forwarding table [75].

A prefix also can be expressed as bit strings of up to 32 bits in IPv4 followed by a "*”. For example, the prefix $1001100011011001^{*}$ represents all the $2^{16}$ addresses that begin with the bit pattern 1001100011011001.

\subsubsection{IPv6 Address Architecture}

Most of today's internet uses IPv4, which is now nearly twenty years old. IPv4 has been remarkably resilient in spite of its age, but it is beginning to have problems. Most importantly, there is a growing shortage of IPv4 addresses, which are needed by all new machines added to the Internet. IPv6 fixes a number of problems in IPv4, such as the 
limited number of available IPv4 addresses. It also adds many improvements to IPv4 in areas such as routing and network auto-configuration. IPv6 is designed to run well on high performance networks (e.g. Gigabit Ethernet) and at the same time still be efficient for low bandwidth networks (e.g. wireless). IPv6 is expected to gradually replace IPv4 in the near future $[86,87]$.

An IPv6 address is 128 bits long and the first few bits of the address define the type and format of the address. It is divided along 16-bit boundaries, and each 16-bit block is converted to a 4-digit hexadecimal number and separated by colons. $21 D A: D 3: 0$ : $2 F 3 B: 2 A A: F F: F E 28: 9 C 5 A$ is an example of a valid IPv6 address. Some types of addresses contain long sequences of zeros. In this case the string of consecutive zeros can be written with the gap separator :: representing the elided string of zeros. But only one gap is allowed in an address. For example, $F F 02: 0: 0: 1: 0: 0: 0: 2$ can be written more compactly as $F F 02:: 1: 0: 0: 0: 2$ or $F F 02: 0: 0: 1:: 2$, but not as $F F 02:: 1:: 2$. When an IPv6 address incorporates an IPv4 address, it can be written in standard IPv4 decimal dot notation. For example $0: 0: 0: 0: 0: 0: 1870: 30 E F$ can be represented as $0: 0: 0: 0: 0: 0: 24.112 .48 .254$ or as $:: 24.112 .48 .254$.

A form similar to the CIDR notation for IPv4 addresses is used to indicate the network part of a IPv6 address. For example, 21DA:D3:0:2F3B :./64 is a 64 bit long prefix, which means that the first 64 bits are the network ID and the other 64 bits are its host ID.

\subsection{Matching Strategies}

In table lookups, we say a packet matches a rule only when all fields match. There are 
4 types of matching required in real lookup processing [26].

Exact match under arbitrary mask The key is $A N D$ with the mask associated with each rule, and then compared with the value for an exact match. The mask bits, 1 's and 0 's, are not necessarily contiguous. We say the key matches a rule, when the value of (rule AND mask) is equal to the value of (rule AND mask). The key 101010 matches the rule 110010 , when the mask is 100110 .

Prefix matching This is a special case of exacting matching under mask. Instead of arbitrary mask, this case guarantees that the mask is a contiguous bits of 1's followed by a contiguous bits of 0 's. IP address lookup belongs to this category, which will be discussed in the next section.

Range matching A lower limit and an upper limit are specified. If the key falls in the range, it is considered a match. TCP port field and ToS byte field belong to this category. Longest prefix matching also is a special case of range matching where the boundary is a power of 2 .

Exact matching This is a special case of all of the above. The key has to match exactly with the field in the rule.

\subsection{One-Dimensional Table Lookup: IP Add ress Lookup}

The primary role of routers is to forward packets to their final destinations. The router must decide for each incoming packet where to send it next [42]. The address of the next-hop router will be obtained and the forwarding engine knows to which output port 
Table 2.1: A Small Sample Forwarding Table

\begin{tabular}{|l|l|l|}
\hline No. & Destination Prefix & Forwarding Information \\
\hline$P_{1}$ & $192.2 .0 .0 / 22$ & $\mathrm{H} 1$ \\
$P_{2}$ & $200.11 .0 .0 / 22$ & $\mathrm{H} 2$ \\
$P_{3}$ & $192.2 .2 .0 / 24$ & $\mathrm{H} 3$ \\
$P_{4}$ & $208.12 .16 .0 / 20$ & $\mathrm{H} 3$ \\
$P_{5}$ & $130.86 .0 .0 / 16$ & $\mathrm{H} 4$ \\
\hline
\end{tabular}

the packet should be forwarded. Such forwarding information is stored in a forwarding table. IP address lookup is to look up the best next-hop router of an incoming packet in the forwarding table.

\subsubsection{Forwarding Table}

A router's forwarding table is usually composed of $<$ Destination Prefix, Forwarding Information >. Destination Prefix is an IP prefix and Forwarding Information is usually a next hop address or an output port number. An example can be seen in Table 2.1. A destination address matches a Destination Prefix if all the significant bits of the prefix are identical to the first few bits of the destination address. An Internet address lookup operation on an incoming packet requires the router to find the most specific router for the packet. For example, the address of an incoming packet, 192.2.2.34, matches two prefix entries, 192.2.0.0/22 and 192.2.2.0/24, in Table 2.1, since those two prefixes overlap. To determine whether $\mathrm{H} 1$ or $\mathrm{H} 3$ is the destination the packet should be sent to, a router must 


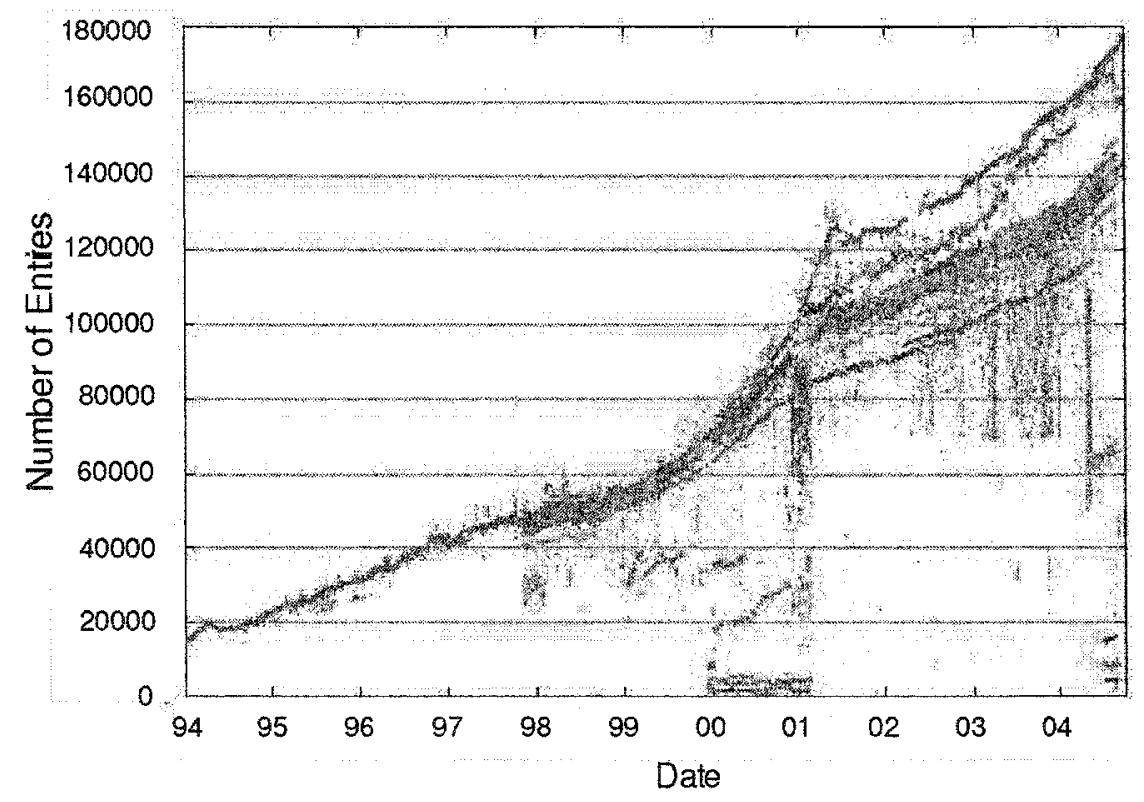

Figure 2.3: Growth of a Forwarding Table

find the most specific match, with the longest matching prefix. In summary, a router needs to solve the Longest (or Best) Matching Prefix (BMP) problem - -a problem of finding the entry containing the longest prefix among all prefixes matching the incoming packet's destination address in a forwarding table.

The growth of the entries in a forwarding table is shown in Figure 2.3 [70]. It shows an exponential growth after mid 1998. Since the number of the entries in a forwarding table is still growing, the address lookup becomes increasingly more difficult.

\subsubsection{Difficulty of Internet Address Lookup}

The longest prefix matching problem is harder than the exact matching problem. The destination address of an arriving packet does not carry with it the information needed to 
determine the length of the longest matching prefix. Hence, we cannot find the longest match search algorithm using an exact match search algorithm (for example, using hash or a binary search procedure). Instead, a search for the longest matching prefix needs to determine both the length of the longest matching prefix as well as the forwarding table entry containing the prefix of this length that matches the incoming packet's destination address. In what follows we will use $N$ to denote the number prefixes in a forwarding table and $W$ to indicate the maximum length of prefixes. Typically $W$ is also the length of the IP addresses.

\subsection{Multi - Dimensional Table Lookup: Packet Classifi- cation}

Traditionally, the Internet provides only a "best-effort" service [21], treating all packets going to the same destination identically, and servicing them in a first-come-firstserved manner. However, the rapid growth of the Internet has caused increasing congestion and packet loss at intermediate routers. As a result, some users are willing to pay a premium price in return for better service from the network. To maximize their revenue, the ISPs also wish to provide different levels of service at different prices to users based on their requirements. In order to provide different services, routers require additional mechanisms, such as admission control, policy routing and traffic accounting. Packets are classified into flows according to some policy or route information. The policy is specified using fields in the header of packets. The specifications of fields are called rules. So flows are specified by rules applied to incoming packets. A collection of rules is 
called a classifier. All packets belonging to the same flow are treated in a similar manner. The identified flow of an incoming packet specifies an action to be applied to the packet. The determination of this action is called packet classification [18].

Packet classification enables a number of additional, non-best-effort network services other than the provisioning of differentiated qualities of service. Some well-known applications of packet classification are firewall, VPN, policy-based routing, traffic ratelimiting and policing, traffic shaping, and billing. These applications use rules based on $\mathrm{L} 2 / \mathrm{L} 3 / \mathrm{L} 4 / \mathrm{L} 7$ fields in the packet header $[4,9,10,11,16]$.

Assume that a $k$-dimensional classifier has $N$ rules. Each rule, $R_{j}, 1 \leq j \leq N$, consists of three entities:

- a regular expression, $R_{j}[i], 1 \leq i \leq k$, on each of the $k$ header fields;

- a number $\operatorname{pri}\left(R_{j}\right)$, including the priority of the rule in the classifier. We follow the convention that the rules closer to the top of the rule list take priority;

- an action, action $\left(R_{j}\right)$.

Table 2.2: The Service and Its Relevant Packet Fields in a Classifier

\begin{tabular}{|l|l|}
\hline Service & Relevant Packet Fields \\
\hline Packet Filtering & Source link-layer address, destination network-layer address \\
Traffic rate limiting & Source link-layer address, source transport port number \\
Traffic shaping & Destination link-layer address, destination transport port number \\
\hline
\end{tabular}


Table 2.2 depicts some examples of services with their relevant packet fields in a classifier [18].

Part of a real-life classifier in 4 dimensions is shown in Table 2.3. The first rule $R_{1}$ has the highest priority and the last rule $R_{4}$ has the lowest priority. The size of a classifier grows fast. There is potential anticipation of classifiers that could support up to one million rules for DiffServ and policing application at edge routers. Even at core routers, classifiers with tens of thousands of rules are also commonly used.

Table 2.3: An Example of a Classifier in Four Dimensions

\begin{tabular}{|l|l|l|l|l|l|}
\hline Rule & $\begin{array}{l}\text { Network-layer } \\
\text { destination prefix }\end{array}$ & $\begin{array}{l}\text { Network-layer } \\
\text { source prefix }\end{array}$ & $\begin{array}{l}\text { Transport-layer } \\
\text { destination }\end{array}$ & $\begin{array}{l}\text { Transport- } \\
\text { layer protocol }\end{array}$ & Action \\
\hline$R_{1}$ & $12.13 .16 .2 / 32$ & $12.13 .80 .1 / 32$ & $*$ & $*$ & Deny \\
$R_{2}$ & $12.14 .3 .0 / 24$ & $12.13 .200 .1 / 32$ & Eq http & Udp & Deny \\
$R_{3}$ & $23.4 .34 .2 / 32$ & $12.34 .0 .0 / 16$ & Gt 1023 & Tcp & Deny \\
$R_{4}$ & $34.35 .32 .0 / 24$ & $4.23 .35 .56 / 32$ & Range 20-21 & Udp & Permit \\
\hline
\end{tabular}

A packet $P$ is said to match a particular rule $R$, for any $i$, the $i^{\text {th }}$ field of the header of $P$ satisfies the regular expression $R[i]$. An incoming packet $P$ with the header can be considered as a $k$-tuple of points $\left(P_{1}, P_{2}, \ldots, P_{k}\right)$. The $k$-dimensional packet classification problem is to find the most specific rule $R_{m}$ with the highest priority among all rules, $R_{j} \mathrm{~s}$, which match the $k$-tuple. We call the rule $R_{m}$ the best matching rule for packet $P$.

Actually, IP address lookup is an instance of one-dimensional packet classification. In this case, all packets destined to the set of addresses described by a common prefix may be considered to be part of the same flow. Each rule has a Destination Prefix as its only 
component and has the next hop address associated with this prefix as the action. If we define the priority of the rule to be the length of the route-prefix, determining the longestmatching prefix for an incoming packet is equivalent to determining the best matching rule in the classifier. The packet classification problem is therefore a generalization of the routing lookup problem.

\subsection{Goals and Metries for Table Lookups Algorithms}

\section{Low storage requirements}

Space Complexity (S) is defined as the asymptotic tight bound $O(f(N))$ on the space required to represent a particular rule database and its associated data structures [19]. For example, a linear search algorithm has a space complexity of $O(N)$. Let $f$ and $g$

be positive functions. The notation $f=O(g)$ means that the ratio $\frac{f(x)}{g(x)}$ stays bounded as $x \rightarrow \infty$.

Low storage requirements enable the use of fast memory technologies like SRAM. On-chip SRAM for semi-custom ASICS is at most 32 Mbits today. Since on-chip SRAM provides the fastest memory access (4-8 nsec), we would ideally develop an algorithm with its memory requirement scale to the size of an on-chip SRAM.

Since the sizes of forwarding tables and classifiers are growing rapidly, and the trend that networks will be migrated from IPv4 to IPv6 is inevitable, thus how to use the limited memory more efficiently becomes more challenging.

\section{High search speed}


Time Complexity (T) is defined as the asymptotic tight bound $O(f(N))$ on the maximum number of steps or cycles that are required to perform a complete lookup. $T$ is used to identify the number of sequential steps required to reach a match/no match result. Any parallelism implemented helps to reduce $T$. Algorithms that narrow down the number of rules to match (and hence the number of steps) would also reduce $T$. For example, a linear search algorithm and a direct table lookup algorithm have time complexity of $O(N)$ and $O(1)$, respectively.

Increasing data rates of physical links require faster address lookups and classification at routers. Table 2.4 illustrates the required lookup speed to meet varied wire-line speed. For example, links running at $\mathrm{OC} 192 \mathrm{c}$ (approximately 10Gbps) rates need the router to process 31.25 million packets per second (assuming minimum-sized 40-byte TCP/IP packets). We generally require algorithms to perform well in the worst case; otherwise, packets would need to be queued before the classification function. At the same time, in some applications, a lookup or classification algorithm that performs well in the average case may be acceptable. Speed is measured in terms of memory accesses. Often a larger memory storage can reduce the number of memory access required. It is not very hard to meet the speed requirement for one application. However, in a network processor, multiple applications are processed in parallel, the key challenge is to make all of the applications meet the processing rate simultaneously.

\section{Efficient power consumption}

Power Complexity $(\mathrm{P})$ is defined as the asymptotic tight bound $O(f(N))$ on the product of the maximum number of steps or cycles that are required to perform classification, the number of operations that are performed in parallel per step, and the cost, in terms of 
Table 2.4: Lookup Speed Table

\begin{tabular}{|c|l|l|}
\hline Year & Line Speed & packetssec (40Byte packet) \\
\hline 1997 & $622 \mathrm{Mb} / \mathrm{s}$ & $1.94 \mathrm{M}$ \\
1999 & $2.5 \mathrm{~Gb} / \mathrm{s}$ & $7.81 \mathrm{M}$ \\
2001 & $10 \mathrm{~Gb} / \mathrm{s}$ & $31.25 \mathrm{M}$ \\
2003 & $40 \mathrm{~Gb} / \mathrm{s}$ & $125 \mathrm{M}$ \\
\hline
\end{tabular}

power required, of a single operation. It attempts to identify the number of basic match operations performed and, by implication, the power required.

For example, a linear search algorithm has a power complexity of $O(N)$. A direct table lookup has a power complexity of $O(1)$.

\section{Fast update speed}

Update Complexity $(\mathrm{U})$ is defined as the asymptotic tight bound $O(f(N))$ on the maximum number of steps or cycles required to perform an atomic insert, delete or update of a rule in the rule database. For example, a linear search algorithm requires an update time of $O(N)$. A direct table lookup algorithm has an update time of $O(1)$.

Since forwarding tables and classifiers change, the entries stored need to be added or deleted frequently. Currently, the Internet has a peak of a few hundred BGP updates per second. Hence, a certain algorithm should be able to perform $1 \mathrm{k}$ updates per second to avoid routing instabilities. These updates should interfere little with normal lookup operations. 


\section{Scalable to different classifiers}

There are many types of classifiers in an NP. The number of the header fields used differs with respect to different applications. We are looking for a general algorithm that can adapt to all of them, so that it makes the industrial implementation easier. Since the migration from IPv4 to $\mathrm{Pv} 6$ is inevitable, the algorithm is expected to be scalable to different IP address architectures.

\subsection{Summary}

In this chapter, we introduced the background of the next generation networks, network processors, and table lookups. We presented the significance of this thesis research area. The goals for the table lookups were also described in the end. 


\section{Chapter 3}

\section{Related Table Lookup Algorithms}

In this Chapter, some existing table lookup algorithms will be illustrated. One-dimensional table lookup (IP address lookup) algorithms will be reviewed in the first, then multidimensional table lookup (packet classification) algorithms will be described next. These algorithms presented are the mainly classical ones, and many novel heuristic algorithms are derived from them.

\subsection{One-dimensional Table Lookup Algorithms - IP Ad- dress Lookup Algorithms}

In this section, we use an example of one-dimensional forwarding table (Table 3.1) with destination prefixes. 
Table 3.1: A Simple One-Dimensional Forwarding Table

\begin{tabular}{|l|l|l|}
\hline & Destination Prefix & Next Hop \\
\hline$P_{1}$ & $01^{*}$ & $\mathrm{H} 1$ \\
$P_{2}$ & $10^{*}$ & $\mathrm{H} 2$ \\
$P_{3}$ & $001^{*}$ & $\mathrm{H} 3$ \\
$P_{4}$ & $111^{*}$ & $\mathrm{H} 4$ \\
$P_{5}$ & $0101^{*}$ & $\mathrm{H} 5$ \\
$P_{6}$ & $1011^{*}$ & $\mathrm{H} 2$ \\
\hline
\end{tabular}

\subsubsection{Simple Schemes}

These simple schemes are straightforward, however, they are impossible for practical use. They can provide the lower bound or the upper bound of the time complexity and the space complexity of an IP address lookup algorithm. The advanced algorithms in the following subsections are developed in order to find a tradeoff between time complexity and space complexity.

\section{Direct Table Lookup}

In the direct lookup algorithm, a forwarding list is created which is as large as the address space. Each IP address itself is an index to the list. The forwarding list is $2^{32}$ long for IPv4 and $2^{128}$ long for IPv6. Only one memory access for a lookup is needed. Update is also as fast as one memory access. Unfortunately, the huge space consumption 
prevents it being used practically. This algorithm reaches an extreme situation of the IP address lookup algorithms: the lower bound of lookup and update complexity, one memory access, and the upper bound of space complexity, $O\left(2^{W}\right)$, where $W$ is the width of an IP address.

\section{Linear Lookup}

The simplest way is to store all prefixes as a linked list in the forwarding table. In a complete lookup, the entire prefixes have to be traversed. Then, the longest matching prefix is reported in the end. Update is also straightforward. It is assumed that there are $N$ entries in the forwarding table. This algorithm reaches another extreme situation of the IP address lookup algorithms: the upper bound of lookup and update complexity, $O(N)$, and the lower bound of the space complexity, $O(N)$. Since, in general, there are tens of thousands of entries in a forwarding table, a linear search is too slow in practice.

\section{Parallel Lookup -Lookups in TCAMs}

Parallel approach can be used to speed up lookup. A TCAM [73] is a storage array with the capability to search the array contents to find the location of a particular stored value. A TCAM is divided into multiple cells. Each element in a cell stores a $W$-bit (val, mask) pair. For example, if $W=6$, a prefix $01 *$ will be stored as the pair $(010000$, 110000). An element matches an input value if those bits of val for which the mask bit is 1 match those in the input. If an element matches the input value the output of the cell is 1 , otherwise it is 0 . It is frequently used in longest prefix match applications.

Figure 3.1 illustrates how a TCAM implements address lookup. The TCAM memory 
bank stores rules in decreasing (increasing) order of priority, and compares the input against each element in the memory in parallel. The output of the memory is an $N$-bit vector, which indicates which rules match the input. Then the priority encoder indicates the address with the highest priority match. The address indexes into a RAM to find the next hop of this prefix.

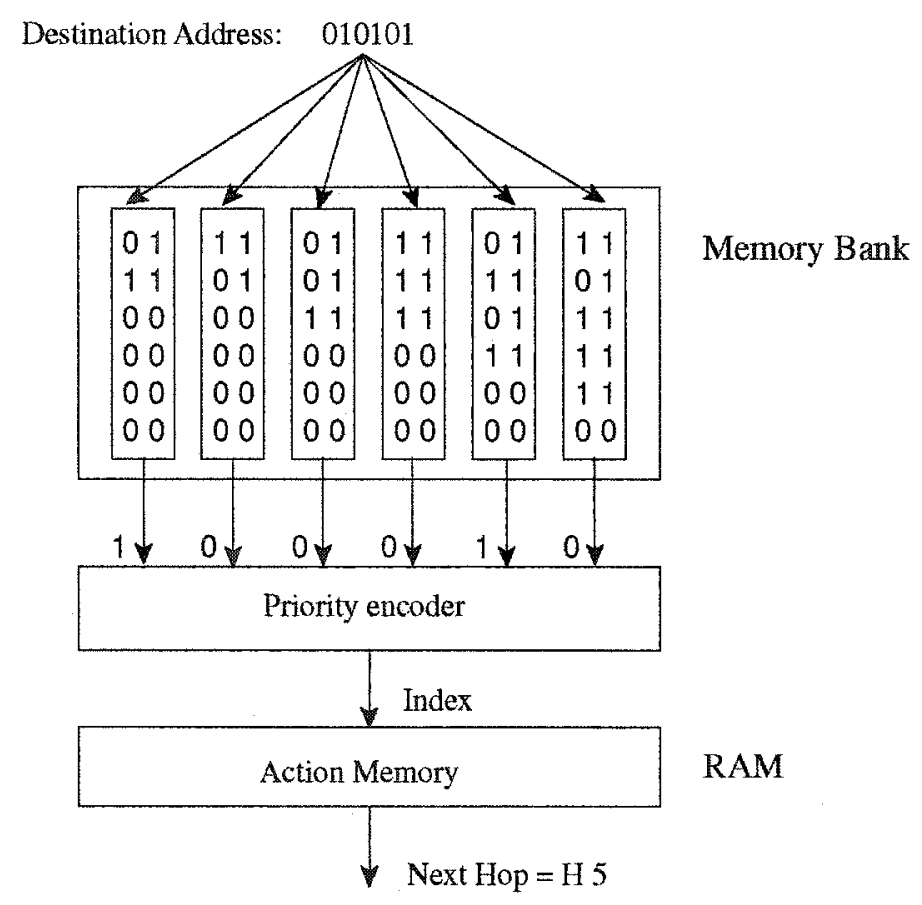

Figure 3.1: Address Lookup in a TCAM

The data storing and retrieving in TCAMs are very fast. TCAMs are increasingly being deployed because of their simplicity and speed. Some venders [74] produce $18 \mathrm{Mb}$ TCAMs that are able to support as many as $512 \mathrm{~K}$ forwarding rules and perform 2 lookups at OC192. Both faster and denser TCAMs can be expected in the near future.

However, its power consumption is much higher than other RAM solutions, because an address is compared against every TCAM element in parallel. Meanwhile, TCAMs 
Table 3.2: Memory Technologies

\begin{tabular}{|l|l|l|l|l|}
\hline Technology & Single chip density & $\begin{array}{l}\$ / \text { chip } \\
(\$ / M B y t e)\end{array}$ & Access speed & Watts/chip \\
\hline Networking & 64 MByte & $\begin{array}{l}\$ 30-\$ 50 \\
(\$ 0.50-\$ 0.75)\end{array}$ & $40-80 \mathrm{~ns}$ & $0.5-2 \mathrm{~W}$ \\
\hline SRAM & 4 MByte & $\begin{array}{l}\$ 20-\$ 30 \\
(\$ 5-\$ 8)\end{array}$ & $4-8 \mathrm{~ns}$ & $1-3 \mathrm{~W}$ \\
\hline TCAM & 1 MByte & $\begin{array}{l}\$ 200-\$ 250 \\
(\$ 200-\$ 250)\end{array}$ & $4-8 \mathrm{~ns}$ & $15-30 \mathrm{~W}$ \\
\hline
\end{tabular}

are more expensive than general RAMs. The update of the rules in a TCAM is a problem, since the rules are stored in the priority order. When adding or deleting a rule, the rules with the lower priorities should be shifted, which has the worst time complexity $O(N)$. Table 3.2 tells us the memory features with respect to different technologies.

\subsubsection{Advanced Algorithms}

\section{Trie based Algorithms}

Compared with the linear approaches, trie based algorithms are hierarchical ones. The classical schemes for the BMP search depend on tries. A set of prefixes in a forwarding table can be represented by a trie. Trie is a tree-based data structure. The branching in 
a trie is based on the bits extracted sequentially from the search key. The nodes with no child are called leaves or external nodes, while the nodes with children are called internal nodes.

(a) Basic Trie - Binary Trie

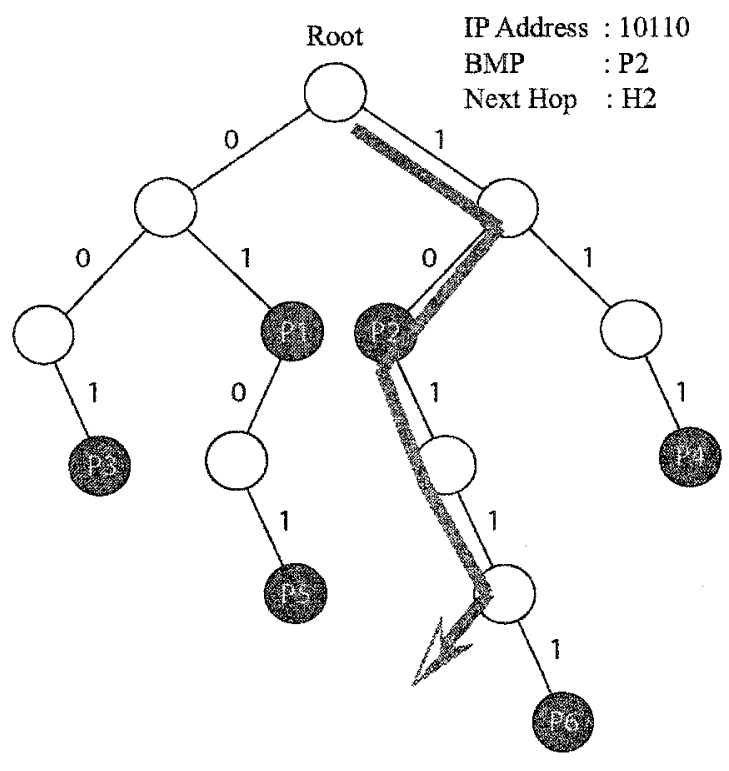

Figure 3.2: A Binary Trie

A binary trie is the simplest type of a trie. Each node has at most 2 children, the branches of which are labeled by 0 (left) or 1 (right). In a binary trie, a node on level $l$ represents the set of all addresses that begin with the sequence of $l$ bits consisting of the string of bits labeling the path from the root to that node. Each dark node represents a prefix in the forwarding table and it also contains further forwarding information (nexthop). White nodes simply guide the search process. Note that every extemal node should be a dark node, however internal nodes may also be dark. 
In an IP lookup, the search in a trie is guided by the bits of the destination address. Once a dark node is traversed, a bucket of memory, which is used to store the BMP and its nexthop, will be refreshed, then a new BMP and its nexthop will be stored. A search ends when there are no more branches.

Although search in a binary trie has to be done bit by bit, the time complexity of which is $O(W)$ in a worst case scenario, it is still a big improvement compared with the complexity of a linear search $O(N)$. From the aspect of space complexity, a binary trie costs $O(N W)$, while a linear search needs $O(N)$. Since the binary trie is a basic trie in the trie family, there are still many ways to improve its time and space performance. Here are some variants of the binary trie.

Figure 3.2 shows a 5 level binary trie representing Table 3.1. Assuming that a destination address is 10110 . When traversing the binary trie to find its nexthop, 5 memory accesses are needed. However, in fact, the result $H 2$ has already been got at the second search step. Since some of the inspection steps are not necessary, a binary trie can be improved to a path-compressed trie.

\section{(b) Path-Compressed Tries}

In general, a binary trie always contains long chains of one-child nodes. In these long chains, there are many internal nodes, which are unimportant for branching decision, and they consume additional space and search time. Path Compression is a scheme to remove those one-way internal nodes, and bit positions are kept in the remaining nodes in order to guide the search correctly. Morrison [34] proposed this path compression scheme first, which is called PATRICIA, but this scheme is not based on BMP matching. Later, Sklower proposed a scheme to combine path compression and BMP [45] implemented 


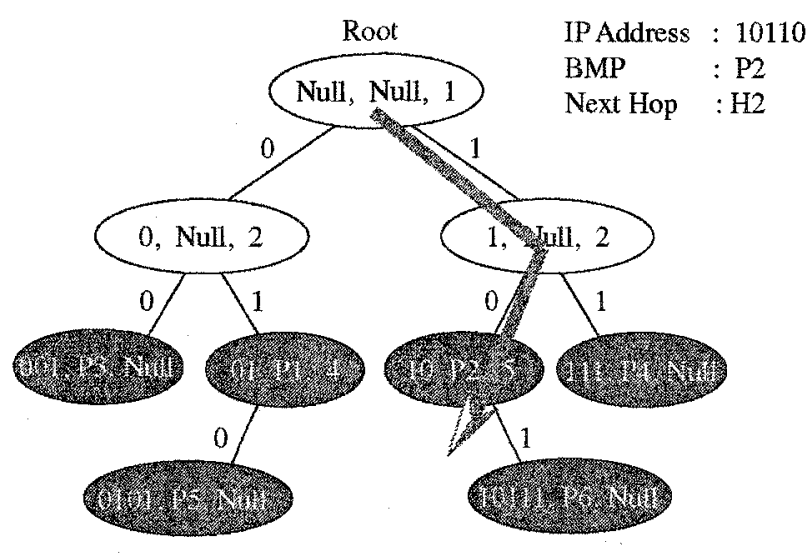

Figure 3.3: A Path Compressed Trie

for the BSD Unix kernel, BSD trie. Each node in the trie contains (Prefix, Nexthop, NextBitPosition) to direct the search. Since some bits are skipped, the Prefix itself must be stored to compare with the key. NextBitPosition is used to indicate the position of the bit in an address to check in the next level. In the worst cases, the time complexity is $O(W)$ and space complexity is $O(N)$.

Figure 3.3 is a 3 level path-compressed trie with respect to Table 3.1. Let the destination IP address be 10110. Looking up in a path-compressed trie shown in Figure 3.3, the memory access times are reduced to 3 in determining its nexthop.

\section{(c) Multibit Tries}

Search in a binary trie is slow since only one bit is inspected at a time. At worst, 32 memory accesses are needed in IPv4. Multibit tries were created to speed up the search operation in order for several bits to be inspected simultaneously. The number of the bits to be inspected per step is called a stride. A stride can be constant or variable, so that in a multibit trie, each node will have $2^{k}$ children and $k$ is the stride. On the other hand, space 


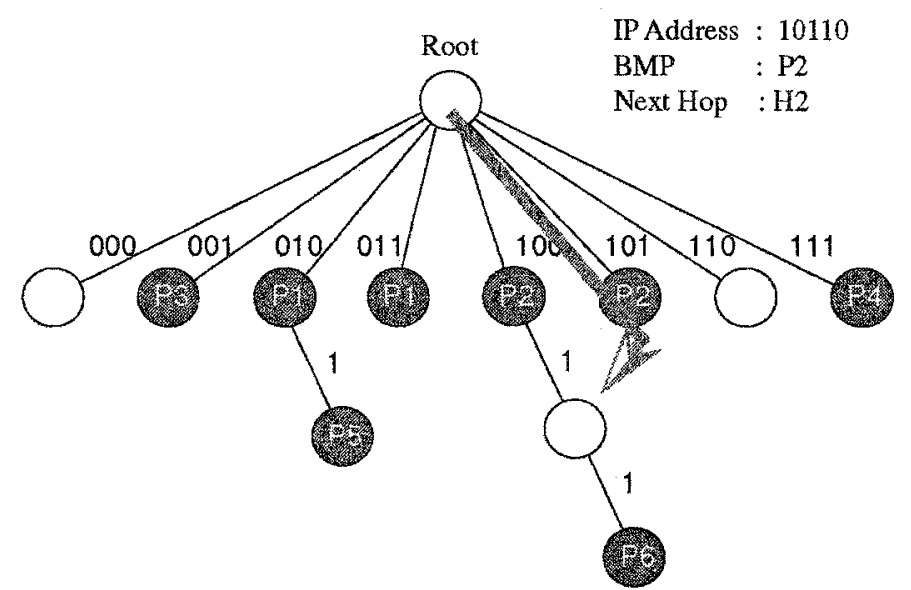

Figure 3.4: An Multibit Trie

complexity in a multibit trie is more than that in a binary trie because of prefix expansion. Therefore a multibit trie is a tradeoff between space consumption and search time. In a worst case scenario, the time complexity is $O(W / k)$ and space complexity is $O\left(2^{k} N W / k\right)$.

Degermark [13] quantified prefix lengths to 3 levels (16, 24 and 32 bits) and expanded every prefix in a forwarding table to the next highest level. The key of this scheme is to let the forwarding table fit into a processor's on-chip cache memory. Srinivasan and Varghese [51] present a data structure by extracting multiple bits at a time and using them as an index into an array of pointers to child nodes. The disadvantage is that it only improves search speeds by a constant factor, but does not scale to longer address lengths.

Examining Figure 3.4, we can see that some leaf nodes are white, since no forwarding information is stored in them. This is a result of the prefix expansion. More memory consumption is expected. Minimizing the number of such "white" leaf nodes in a multibit trie is always a rule to control memory consumption. Let the destination IP address be 10110. Only 1 memory access is needed to figure out its nexthop from Figure 3.4. 
Nilsson and Karlesson [37] proposed a new approach called a level-compressed (LC) trie. LC tries combine the merits of the path-compressed trie and the multibit trie. Both the hight and the nodes of the multibit trie are reduced.

\section{Range based Algorithms}

Consider two distinct intervals $\mathcal{A}$ and $\mathcal{B}$. Their relationship can be classified into three categories:

- disjoint, when $\mathscr{A} \cap \mathcal{B}=\emptyset$;

- overlap, when $\mathcal{A} \cap \mathcal{B} \neq \emptyset$, but $\mathcal{A} \not \subset \mathcal{B}$ and $\mathcal{B} \not \subset \mathcal{A}$;

- covered, when $\mathcal{A} \subset \mathcal{B}$ or $\mathcal{B} \subset \mathcal{A}$.

A prefix stands for a set of contiguous addresses. For instance, supposing 4-bit-length addresses, prefix $a=1^{*}$ defines a interval of addresses $[8,16)$. Two such prefix based intervals are either disjoint or covered. Since longer prefixes represent shorter intervals, a search for the BMP is equivalent to finding the narrowest enclosing interval of the point represented by the address.

\section{(a) Binary Search Tree}

Figure 3.5 shows the search tree of Table 3.1. All the nodes correspond to the left endpoints and each leaf stores BMP. The search branch is guided by the comparison between the address and value in the node. If the address is smaller, we track the left branch, otherwise we go to the right. The time complexity is $O(\log (2 N))$, and space consumption is $O(N)$. 


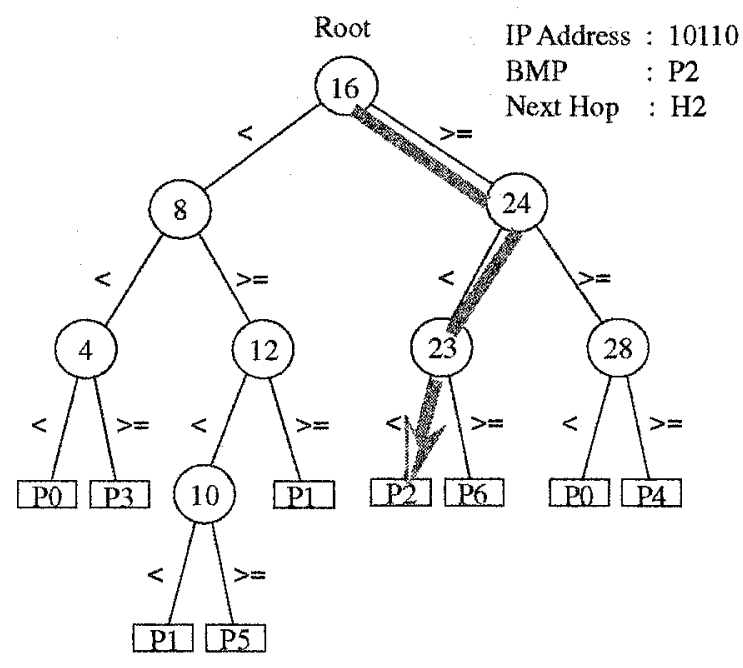

Figure 3.5: A Binary Search Tree

\section{(b) Multiway Search Tree}

Multiway search trees are used to reduce the height of a tree so that it can improve the search time, which is similar to a multibit trie. Lampson described this idea in [32], in which he took advantage of cache line size to do a multiway search with six-way branching, and extended it for IPv6. The search complexity is $O(\log (2 N))$, and space consumption is $O(N)$, where $k$ is the number of branches at each node of the search tree.

\section{Hash Based Schemes}

Hash is a process to access a record by mapping a key value to a position in a table. Hash function $h$ is a function that maps key values to a position. Figure 3.6 gives us a simple hashing example. The domain region of $x$ is $\{1,2,3,4,5,6,7,8\}$, and $h$ is denoted by $h(x)=(x \bmod 3)$. Therefore, the range of $y=h(x)$ should be $\{0,1,2\}$. Table $X$ is mapped into a smaller table $Y$. 


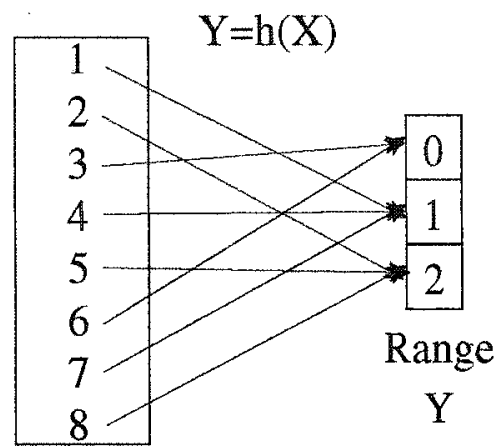

Domain

$\mathrm{X}$

Figure 3.6: A Simple Hash Function

Hash is an exact matching method. Collisions may occur sometimes. The target of hash in lookup is to map all prefixes into $M$ buckets. Then in each bucket, a linear search, trie based or range based scheme can be used. The advantage of using hash is to narrow down the search range sharply. In order to improve its performance, a suitable hash table is required. It ensures that the number of items that fall in a bucket corresponds to the capacity of a singe cache line, so that each hash bucket corresponds to a cache line of memory. Binary Search on Prefix Lengths is an example of IP address lookup based on hash functions.

A forwarding table is decomposed into $M$ hash tables with respect to prefix lengths. In each hash table, the corresponding number of first bits is extracted out from the incoming address to compare with the prefixes. During lookup, a linear search on the space of lengths requires traversing each of the $M$ hash tables. The memory access complexity is $O(M)$. Figure 3.7 shows a hash table according to the prefix lengths with respect to the forwarding table in Table 3.1 .

In order to reduce the search time, a binary search on lengths was proposed by Wald- 


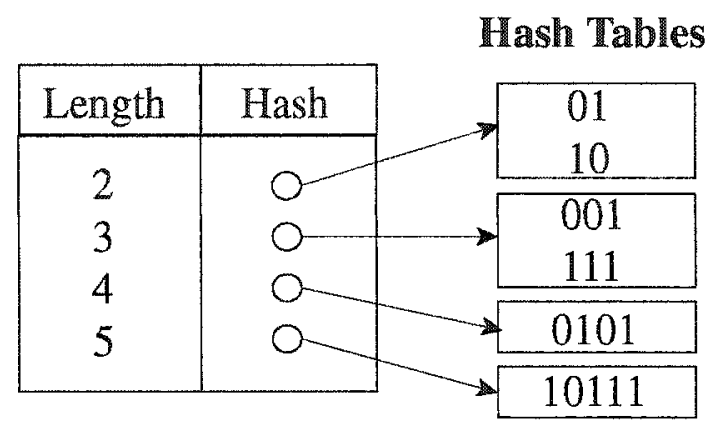

Figure 3.7: Hash Tables for Each Possible Prefix Length

vogel [60]. In a binary search, the search space in each step is reduced by half. The comparison results (if a match can be found) on each level will guide its branching decision. If a match is found, the search space will be the longer length sets. Waldvogel also added extra prefixes of adequate length, called markers, to be sure that when no match is found the search must proceed in the direction of shorter prefixes. The memory access complexity is $O(\log M)$ and space consumption is $O(N \log M)$. Figure 3.8 shows a binary search tree and hash tables including markers based on the forwarding table in Table 3.1.

\subsection{Multi-Dimensional Table Lookup Algorithms - Packet}

\section{Classification}

Table 3.3 is a sample of a 2-dimensional classifier with ten rules. All field specifications are prefixes of maximum length 3 bits. Rule priorities are ordered in decreasing order from top to bottom of the classifier. 


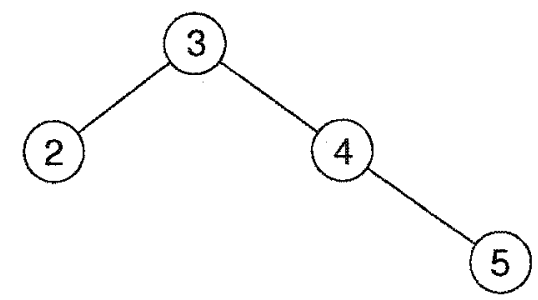

(a) Binary Search Tree

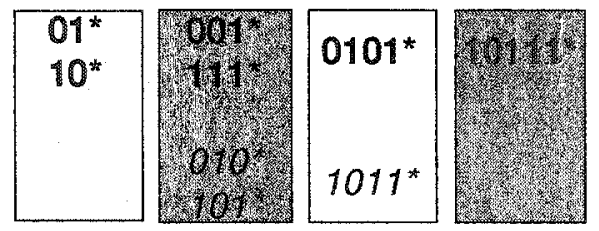

(b) Hash Tables with Markers

(Bold: Prefixes, italic: Markers)

Figure 3.8: Binary Search Tree and Hash Table

\subsubsection{Geometric Representation}

We have already known that a prefix represents a contiguous interval on the number line. Similarly, a 2-dimensional rule represents an axes-parallel rectangle in the 2dimensional Euclidean space of size $2^{\left(W_{1}+W_{2}\right)}$, where $W_{1}$ and $W_{2}$ are the respective widths of the two dimensions. Generally, a rule in $d$ dimensions represents a $d$-dimensional hyper rectangle. A classifier is therefore a collection of super-volumes, each of which is labeled with a priority. An incoming packet header represents a point with coordinates equal to the values of the header fields corresponding to the $d$ dimensions. For example, Figure 3.9 depicts the geometric representation of the classifier in Table 3.3. Given this 
Table 3.3: A Sample of Two-Dimensional Classifier

\begin{tabular}{|l|l|l||l|l|l|}
\hline Rule & Field 1 & Field 2 & Rule & Field 1 & Field 2 \\
\hline R1 & $000^{*}$ & $000^{*}$ & R6 & $0^{*}$ & $11^{*}$ \\
R2 & $000^{*}$ & $011^{*}$ & R7 & $01^{*}$ & $0^{*}$ \\
R3 & $10^{*}$ & $11^{*}$ & R8 & $11^{*}$ & $1^{*}$ \\
R4 & $10^{*}$ & $10^{*}$ & R9 & $1^{*}$ & $0^{*}$ \\
R5 & $1^{*}$ & $01^{*}$ & R10 & $0^{*}$ & $*$ \\
\hline
\end{tabular}

geometric representation, classifying an arriving packet is equivalent to finding the highest priority rectangle among all rectangles that contain the point, which is used to represent the packet. For example, the packet represented by the point $\mathbf{P}(111,010)$ in Figure 3.9 is classified to rule $R 5$ (assuming rules are arranged in non-increasing priority).

\subsubsection{Trie Based Algorithms}

\section{Basic Trie of Tries}

A $d$-dimensional hierarchical trie is a simple extension of the one-dimensional trie data structure. We first build a 1-dimensional trie, F1 trie, with respect to the rules in Field 1. For each prefix, $p$, in the $\mathrm{F} 1$ trie, we recursively build a $(d-1)$-dimensional hierarchical trie on the rules which specify exactly $p$ in dimension $F 1$. The storage complexity of the data structure for an $N$-rule classifier is $O(N d W)$. 


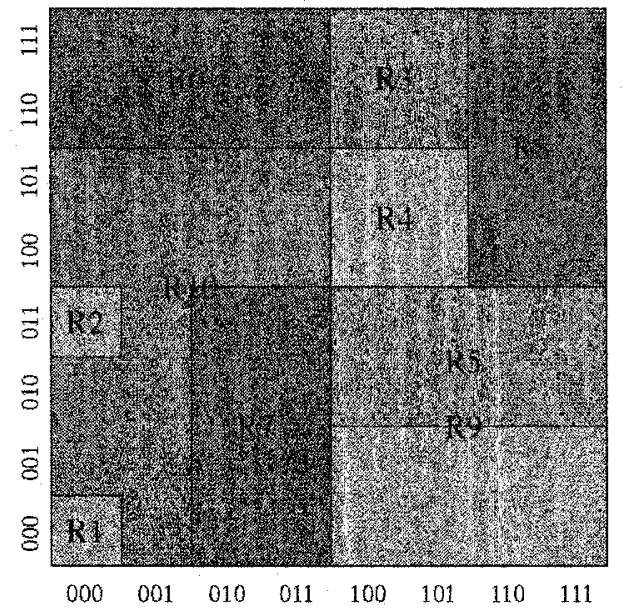

Figure 3.9: Geometric Representation of the Two-Dimensional Classifier of Table 3.3

Finding the best matching rule involves searching all possible paths in the hierarchical tries. Since in one dimension there are many possible matching rules, backtracking in each trie is needed to make sure all the possible nodes are checked. After scanning the trie of tries, a set of rules is obtained and we can find out the best matching rule from them. For instance, if there is an incoming packet $(000,110)$, the searching paths are shown in Figure 3.10 with respect to the classifier in Table 3.3. The time complexity is $O\left(W^{d}\right)$.

\section{Grid of Tries}

The grid of tries scheme [52] is proposed for two-dimensional classification, the key idea is to use precomputation and store a switch pointer in some nodes to speed up the search in the Field 2 trie based on the search in the Field 1 trie. The switch pointer is labeled 0 or 1 and guides the search process. This scheme not only avoids the memory blowup of the set-pruning trie but also avoids the backtracking of the ancestors in the basic trie of tries. 


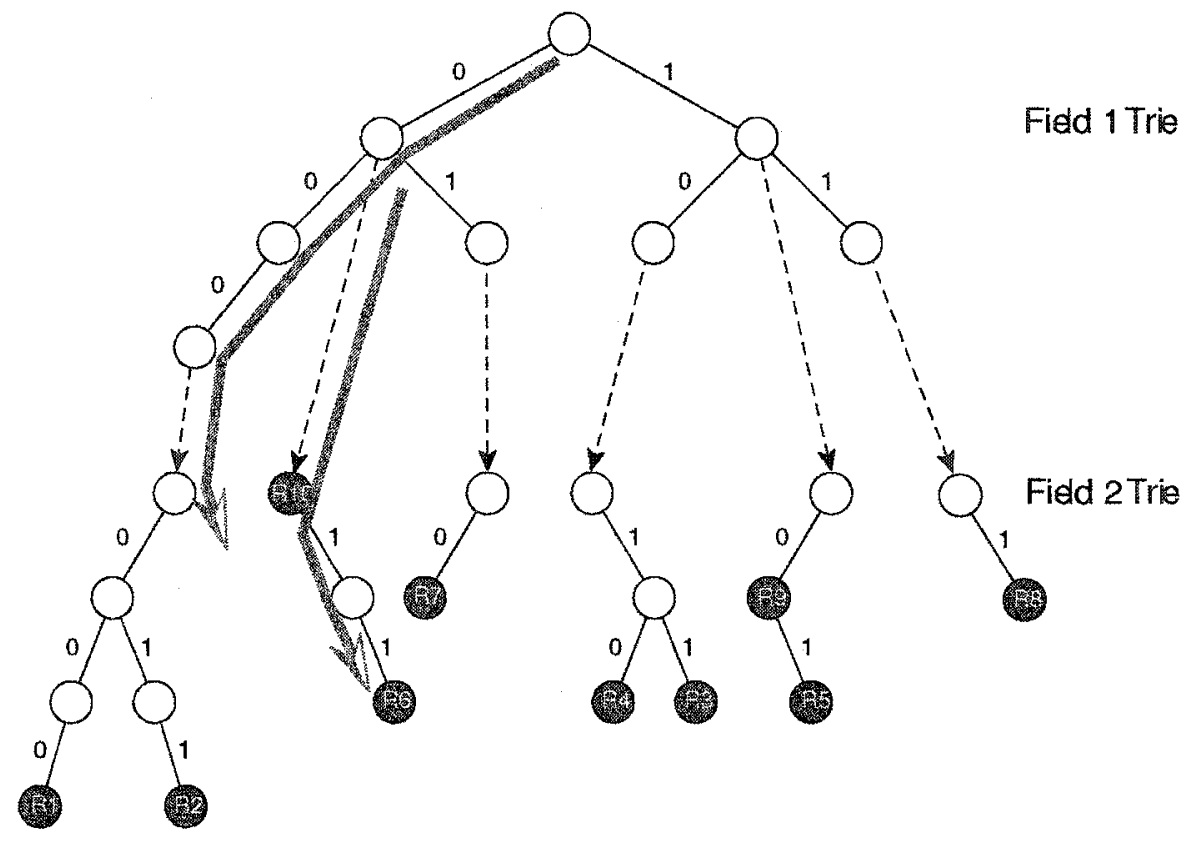

Figure 3.10: Basic Trie of Tries

For example, in Figure 3.11, assume that we are searching $(101,011)$. In the Field 1 trie, the longest matching prefix is $10^{*}$. In the Field 2 trie, we fail to search 011 at the node $A$. Instead of backtracking, we place a switch point at node $A$. With the help of the switch point, the best matching rule $R 5$ is found successfully. However, switch points can only be used for two-dimensional classifiers. Therefore, the time complexity for $d$ dimensions is only reduced to $O\left(W^{d-1}\right)$.

\section{Aggregate Bit Vector Search}

The aggregate bit vector scheme proposed in [4] is a form of divide-and-conquer. It divides the packet classification problem into $d$ sub-problems, and then combines the results. $d$ 1-dimensional tries according to each dimension in the original database are 


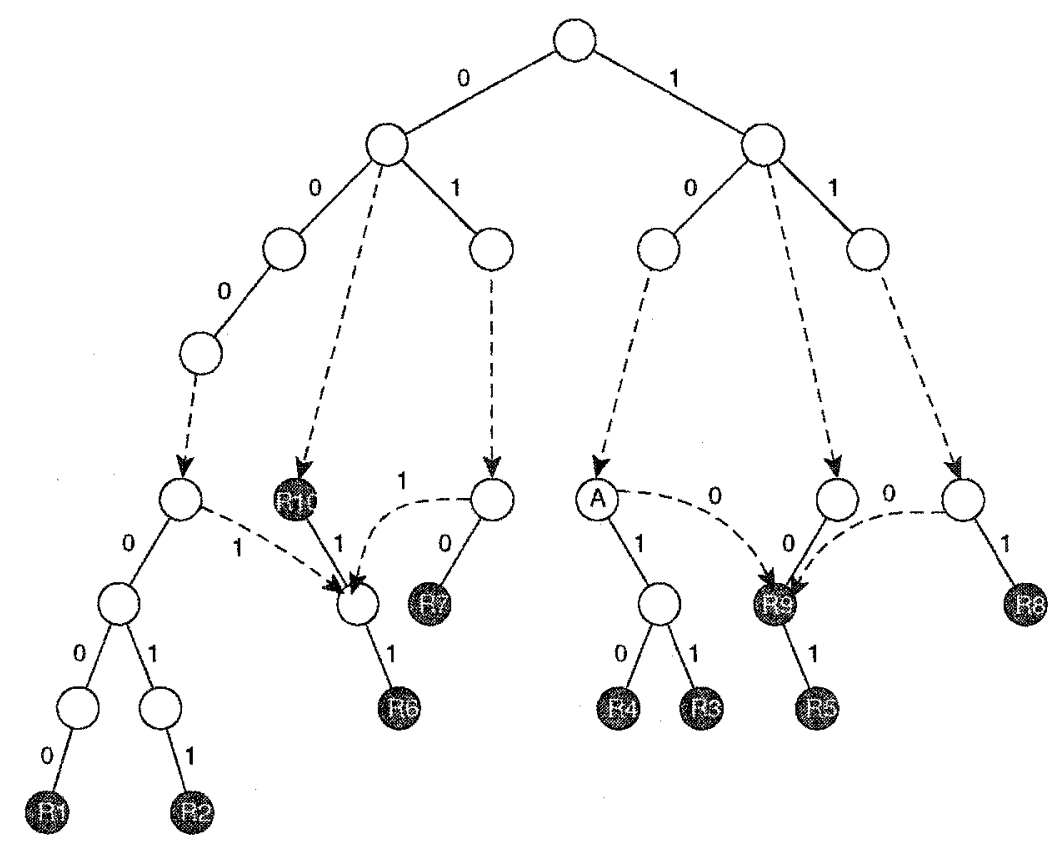

Figure 3.11: Grid of Tries With the Use of Switch Pointers

built at first. An $N$-bit vector is associated with each node of the trie corresponding to a valid prefix, where $N$ is the length of the classifier. Each node in the trie for a field is labeled with an $N$-bit vector. Bit $j$ in the vector is set to 1 if the prefix corresponding to rule $R_{j}$ in the classifier matches the prefix corresponding to the node. Additionally, another vector, called the aggregate bit vector, appends the bit vector for each field in each trie. The aggregate size is $m$, which is a constant that can be tuned to optimize the performance of the aggregate scheme. A bit $i$ is set to 1 in the aggregate vector if and only if there is at least one bit $k$ in the bit vector is set to $1, k \in[i \times m,(i+1) \times m]$. The aggregate bit vector has $\left\lceil\frac{N}{m}\right\rceil$ bits.

When a packet header arrives with fields $\left(P_{1}, P_{2}, \cdots, P_{d}\right)$, we search for the longest matching prefix, $M_{i}$, in each field $i$ to get and read the resulting bit vectors and aggregate bit vectors from each trie. By examining the top level aggregate bit maps; we then only 

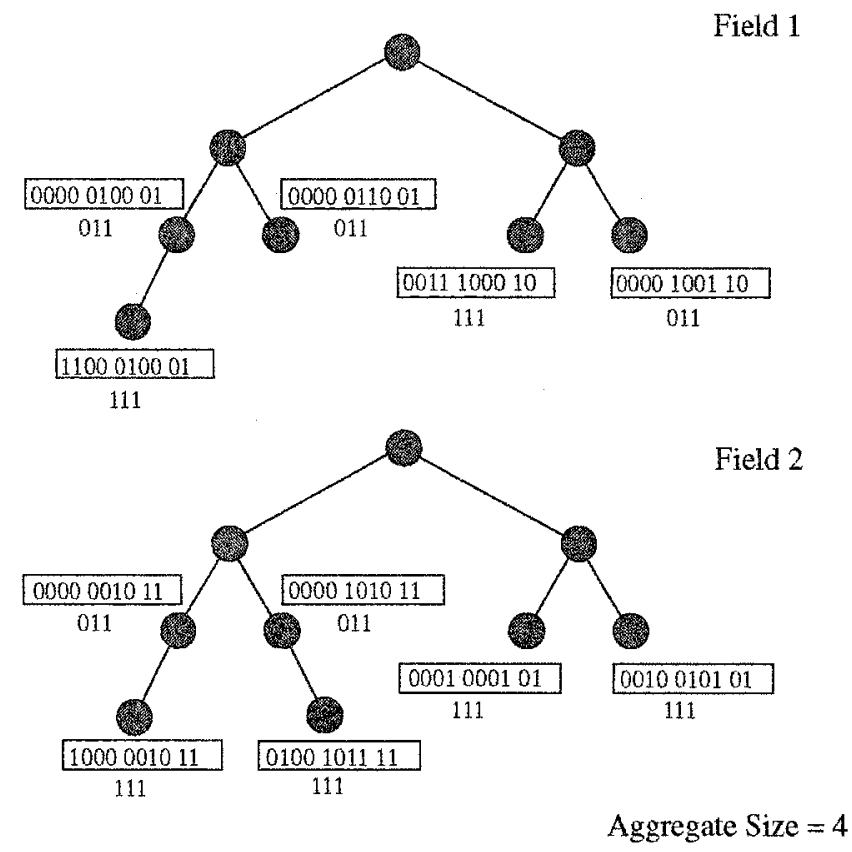

Figure 3.12: Two Tries Associated With Each of the Fields In Table 3.3, together with both the bit vectors and the aggregate bit vector. The aggregate bit vector has 3 bits using an aggregation size of 4 .

examine the leaf bit map values for which the aggregate bits are all set to 1 . If the rules are arranged in non-decreasing order of cost, all we need to do is to find the index of the first bit set in all the bit vectors.

Figure 3.12 illustrates the construction for Table 3.3 using an aggregate size $m=4$. Let us consider an incoming packet $(0010,1101)$. The longest prefix match is 00 in field 1 and 11 in field 2. The associated bit vectors are 0000010001 and 0010010101 while the aggregate ones are 011 and 111. The $A N D$ operation on the two aggregate vectors yields 011 , showing that a possible matching rule must be located only in the last 6 bits. Thus it is not necessary to retrieve the first 4 bits for each field. 


\subsubsection{Comprehensive Search Algorithms}

\section{I Área-based Quadtree}

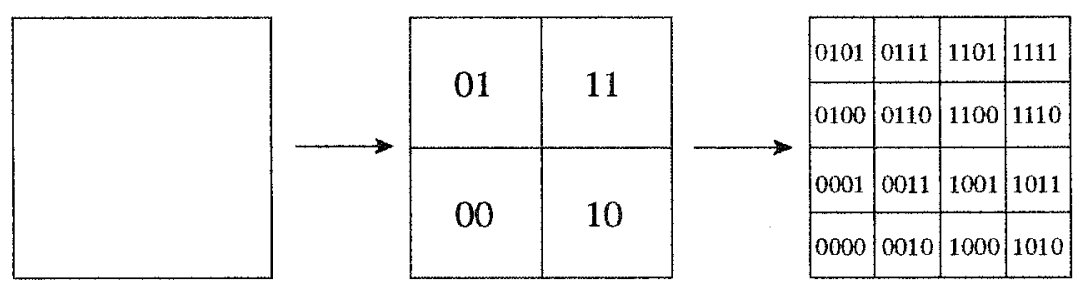

Figure 3.13: Decomposing the Space Recursively.

The area-based quadtree (AQT) [6] was proposed by Buddhikot for two-dimensional classification. Quadtree is a data structure representing the recursive decomposition of the whole space into quadrants. Figure 3.13 illustrates the decomposition of the space.

Each node of a quadtree represents a 2-dimensional space that is decomposed into four equal-sized quadrants, each of which is represented by a child node. The 2-dimensional space is recursively decomposed into four equal-sized quadrants till each quadrant has at most one rule in it or all the rules contained are crossing the quadrant. A rule is said to cross a quadrant if it completely spans at least one dimension of the quadrant. Figure 3.14 shows an example based on Table 3.3.

To perform a packet classification, we extract the values from the both fields of the header. The values are represented as digital bits. We successively choose bits from each value to form a decision branch. The search will finish when we reach a leaf of the quadtree. An efficient update algorithm that has $O(N W)$ space complexity was proposed 
in [6]. $O(\alpha W)$ search time and $O\left(\alpha^{\alpha} \sqrt{N}\right)$ update time, where $\alpha$ is a tunable integer parameter.

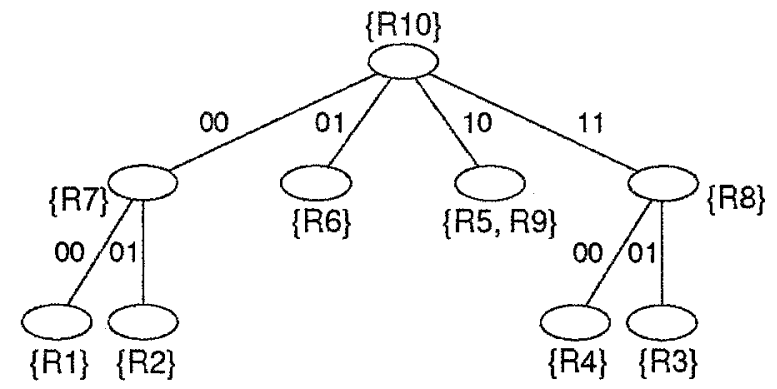

Figure 3.14: Decomposing the Space Recursively.

\section{Tuple Space Search}

A tuple space search algorithm [49] maps all the rules into tuples according to the length of the prefixes in each dimension. The tuple $T(a, b)$ represents the set of rules having $a$-bit long prefixes in the first field and $b$-bit long prefixes in the second field. Since all the rules in one tuple possess the same prefix length in each field, the hash table can be used to search the exact matching rule with the concatenation of prefixes bits as the hash key. For example, if the incoming packet is $P=\left(p_{1}, p_{2}\right)$, we can use the first $a$ bits of $p_{1}$ and the first $b$ bits of $p_{2}$ as the hash key to search the hash table for the tuple $T(a, b)$. Note that at most one matching rule can be found when searching in a tuple.

Finding the best rule involves several hash table probes in all possible tuples. Query time is the total time of a number of hash table accesses. While the number of possible tuples is large ( $W \times W$ for two dimensions), some precomputations of marks and rectangle searches are used to guide the probe procedure and reduce the number of probed tuples. 
In a two-dimensional lookup algorithm, the memory complexity and the search complexity of a tuple space search are $O(N \sqrt{W})$ and $O(W)$ respectively. Incremental update is also supported by tuple space searches.

\section{Recursive Flow Classification}
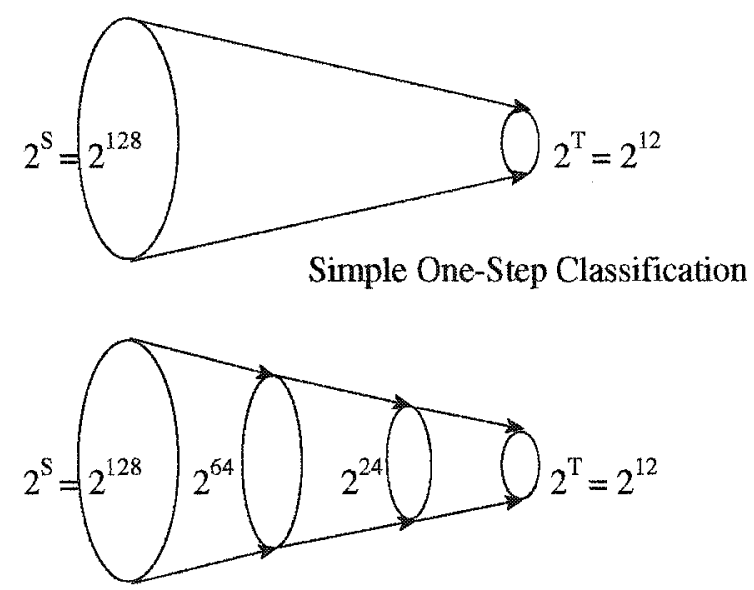

Phase 0 Phase 1 Phase 2 Phase 3

Recursive Classification

Figure 3.15: The Mapping of $S$ Bits to $T$ Bits

The multi-filed packet classification problems can be viewed as mapping an $S$-bit packet header to a $T$-bit action identifier, where $T=\log N, T \ll S$. The simplest but impractical method is to pre-compute the actions of all possible $2^{S}$ different packet headers, yielding the action in one step. When a packet comes, it can be indexed in one memory access. The Recursive Flow Classification (RFC) algorithm [16] takes the same mapping principle but it performs several phases of mapping, shown in Figure 3.15.

Figure 3.16 illustrates the architecture of the RFC algorithm. In the first phase, the $d$ 


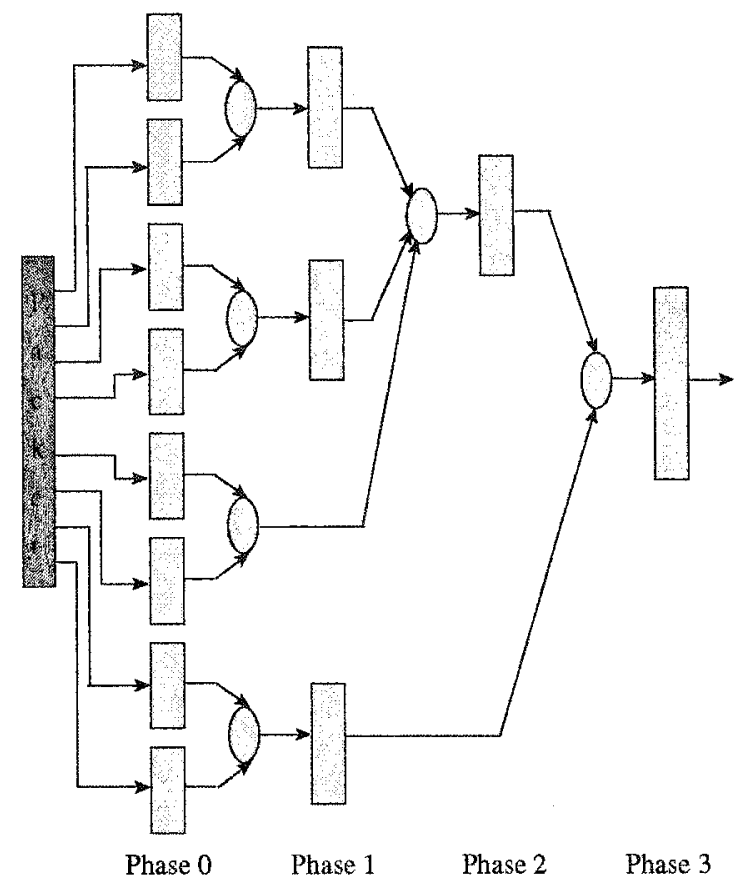

Figure 3.16: The Packet Flow in RFC

fields of the packet header are split up into a few chunks, so that they are used to index into multiple memories in parallel. The contents of each memory are chosen so that the result of the lookup is narrower than the index. In the subsequent phases, memories are indexed using the results from earlier phases. In the final phase, the memory yields the action.

The RFC algorithm requires a lot of pre-computations of the content of the memory blocks. The dynamic incremental update is difficult, since the insertion or deletion causes the reconstruction of all the tables. It is only suitable for relatively static classifiers.

\section{Hierarchical Intelligent Cuttings (HiCuts)}




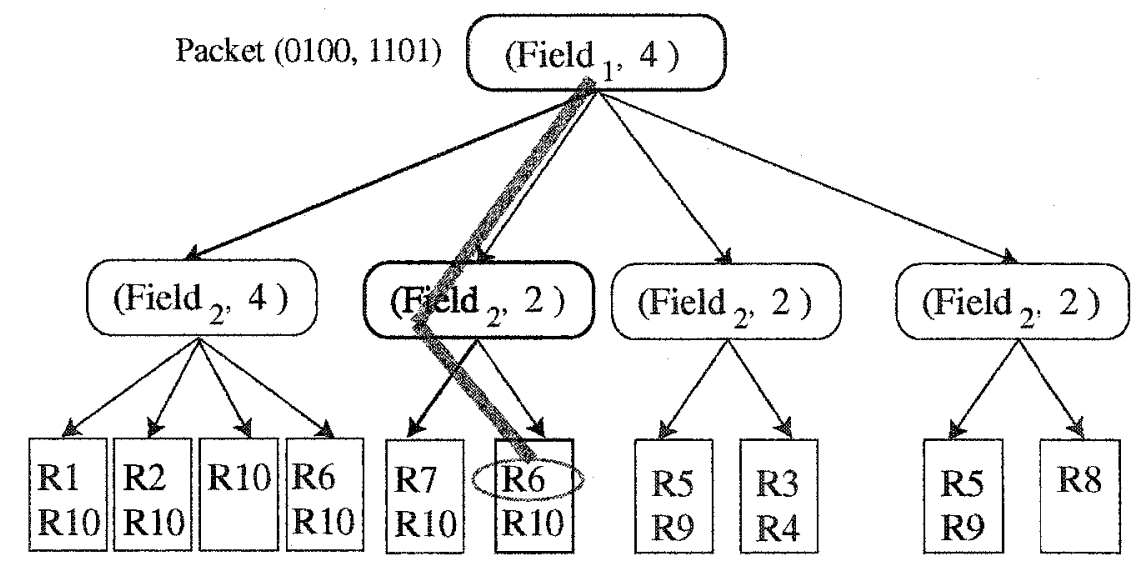

Figure 3.17: The Structure of HiCuts for Table 3.3

HiCuts [17] partitions the multidimensional search space guided by heuristics that exploit the structure of the classifier. Each query leads to a leaf node in the decision tree, which stores a small number of rules that can be searched sequentially to find the best match. The characteristics of the decision tree (its depth, degree of each node, and the local search decision to be made at each node) are chosen while preprocessing the rule set based on its characteristics.

Each node of the tree represents a portion of the geometric search space. The root node represents the complete $d$-dimensional space, which is partitioned into smaller geometric subspaces, represented by its child nodes, by cutting across one of the $d$ dimensions. Each subspace is recursively partitioned until no subspace has more than $B$ rules, where $B$ is a tunable parameter of the preprocessing algorithm.

An example is shown in Figure 3.17 for Table 3.3 with $B=2$. Given a packet $(0100$, 1101), the query first traverses the tree according to the proper bits in the header, until a leaf node is reached. The best matching rule is obtained by a linear search among the rules stored in the leaf node. 


\subsection{Summary}

In this chapter, we outlined some of the classical IP address lookup algorithms and packet classification algorithms in recent years. We tried to master the essence of these algorithms rather than the details of the techniques. It is important to understand the benefit and drawbacks of those algorithms, since most novel techniques are variants of these classical algorithms. 


\section{Chapter 4}

\section{IP Address Lookup Based on CES}

\subsection{Introduction}

There are many lookup algorithms based on the binary trie or index table[45]. The lookup in a binary trie is a simple sequential algorithm. The number of memory access times (32 for IPv4) is much higher than what we expected. Since NPs support parallel processing, it is possible to speedup IP address lookup by converting a single sequential process to multiple parallel small sequential processes. Then, let's consider the index table lookup. It achieves the lower bound on the search time, but its memory consumption is too huge. The row length of an index table depends on the length of the addresses (sequences). If an index table is built with respect to $M$-bit addresses, then the index table is $2^{M}$ long. However when the address is reduced to $\frac{M}{2}$ bits long, the length of the index 
table will be decreased to $2^{\frac{M}{2}}$ sharply, which is one out of $2^{\frac{M}{2}}$ of the former table. When an $M$-bit address based index table is divided into a pair of $\frac{M}{2}$-bit address based index tables, the memory consumption will be reduced. Therefore, we are investigating an approach that is able to take advantage of the parallel processes to improve the binary trie lookup algorithm and the index lookup algorithm.

In this chapter, an efficient IP address lookup algorithm and its architecture will be presented. In our proposed method, one forwarding table is decomposed into two balanced extended smaller sub-forwarding tables by a novel splitting rule. The sub-forwarding tables are said to be "extended", since the forwarding information of each sub-rule contains not only nexthops but also some additional information. Therefore, an IP lookup process can be converted into a pair of small sub-lookups. After the matching sub-prefixes from each sub-forwarding table are got, their attached forwarding information will be compared to determine the final nexthop of the incoming packet. The sub-lookups and information comparison can be processed in parallel. Our approach not only speeds up the BMP search, but also reduces storage requirement.

The main contribution of the proposed algorithm is two-fold. Firstly, a new technique, called Comb Extraction Scheme (CES), is created to transform one forwarding table into a pair of balanced sub-tables. The pair independent sub-lookup processes can be processed simultaneously. In order to determine the output port for a packet, the comparison, between the matching sub-prefixes from a sub-table with the ones from the other, needs to be carried out at the end. $\mathbb{P}$ address lookup using CES can reduce storage space and speed up search time. Secondly, two efficient architectures and their associated search strategies are also proposed. The flexible architectures allow IP address lookup to be easily integrated within routing SoCs and generic network packet processing units. 
In this algorithm, we focus on the unicast (single-source, single-destination) routing of backbone routers. In a forwarding table, an entry usually has this structure: < DesPrx,PrxLen,ForInf $>$. DesPrx is the Destination Prefix, PrxLen is the length of the prefix, and ForInf usually is a next hop address or an output port number. When examining the forwarding tables carefully, we can find that the number of distinct next hops in a forwarding table is very small compared to the tens of thousands of prefixes. It is shown clearly in sample Table 4.1 (only 3 ports for 16 entries). All the entries are sorted in terms of the ports. The index is a number to distinguish between entries sharing the same port.

Table 4.1: A Sample Forwarding Table

\begin{tabular}{|l|l|l|l||l|l|l|l|}
\hline Index & Prefix & Len & Port & Index & Prefix & Len & Port \\
\hline 1 & $11000110100110^{*}$ & 14 & 1 & 5 & $100010101011^{*}$ & 12 & 2 \\
2 & $11000110011^{*}$ & 11 & 1 & 6 & $1110111101^{*}$ & 10 & 2 \\
3 & $11000110010^{*}$ & 11 & 1 & 7 & $01111000101^{*}$ & 11 & 2 \\
4 & $1101011100001011^{*}$ & 16 & 1 & 8 & $0111100011100^{*}$ & 13 & 2 \\
1 & $1100011001^{*}$ & 10 & 2 & 1 & $1100011010011010^{*}$ & 16 & 3 \\
2 & $011110000011^{*}$ & 12 & 2 & 2 & $1100011001110^{*}$ & 13 & 3 \\
3 & $11000110100^{*}$ & 11 & 2 & 3 & $1000101011001^{*}$ & 13 & 3 \\
4 & $10001010110011^{*}$ & 14 & 2 & 4 & $01111000001110^{*}$ & 14 & 3 \\
\hline
\end{tabular}




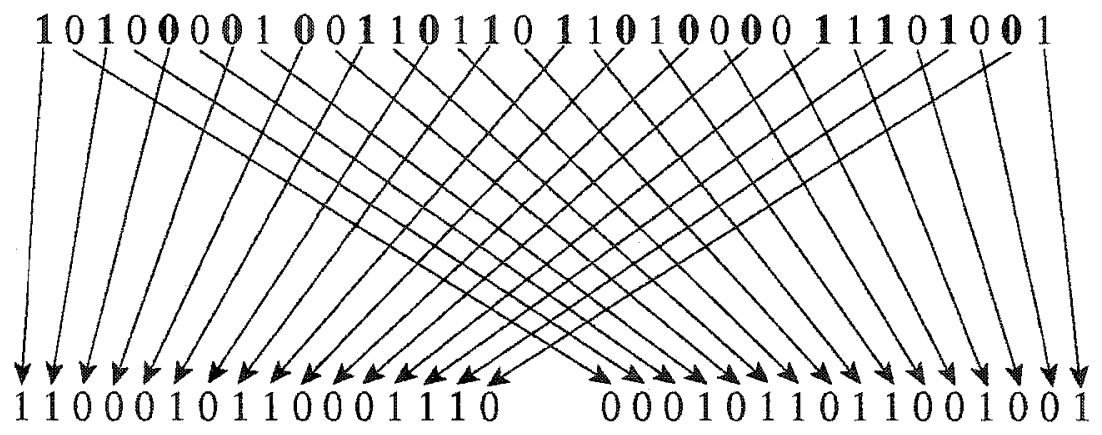

(a) Decomposition of an IP Address by using CES

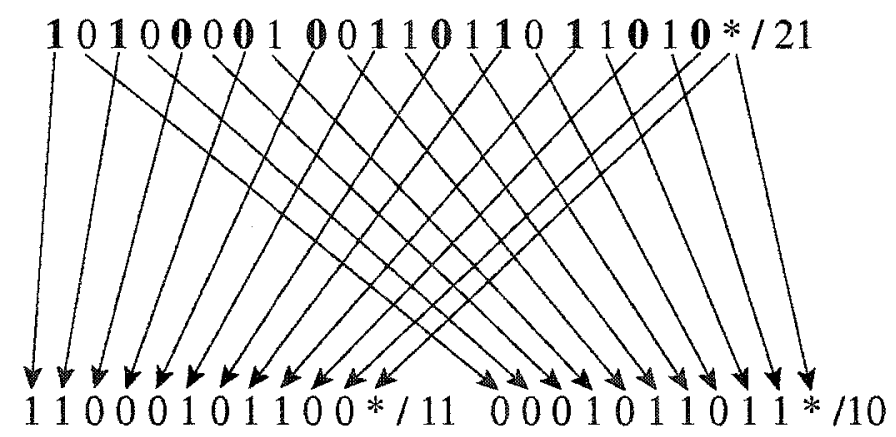

(b) Decomposition of a Prefix by using CES

Figure 4.1: Examples of Using CES

\subsection{New Data Structure}

\subsubsection{Comb Extraction Scheme (CES)}

In IPv4, an IP address $P$ is 32-bit long. It can be decomposed into two 16-bit long 
sub-sequences by the following strategy: From the left-most bit to the right-most bit, all the bits in the odd positions are extracted to form sub-sequence $\alpha$, and all the bits in the even positions are extracted to form sub-sequence $\beta$. We call this splitting rule the Comb Extraction Scheme (CES). For example, consider the following IP address in binary mode, 10100001001101101101000011101001 , in which bits in odd positions are bold. After the decomposition, $\alpha$ and $\beta$ will be 1100010110001110 and 0001011011001001 , respectively.

Similarly, a prefix also can be decomposed into two sub-prefixes $\alpha$ and $\beta$, both of which end by the wildcard *. In short, $\alpha$ and $\beta$ are two sub-prefixes. For example, let us consider the prefix 101000010011011011010*, the length of which is 21. After the decomposition, $\alpha$ is $11000101100^{*}$, the length of which is 11 , and $\beta$ is $0001011011^{*}$, the

length of which is 10. CES provides us with two sub-prefixes of (almost) equal length, no matter how long the original prefix is.

\subsubsection{The Decomposition of a Forwarding Table}

A forwarding table can be converted into two extended sub-forwarding tables by using CES. Table 4.2 and Table 4.3 are the pair of sub-forwarding tables corresponds to the entries in Table 4.1. Each entry in the sub-forwarding table has the structure as $<S u b$ Prefix, Length, Port Indicator, Forwarding Information>.

In Table 4.2 and Table 4.3, the Forwarding Information is composed of a set of forwarding units. Each forwarding unit is a combination of a port number and an index number from Table 4.1. One forwarding unit can be expressed as $a(b)$, where $a$ stands for the output port that its original prefix can be forwarded to and $b$ stands for the correspond- 
Table 4.2: Sub-Forwarding Table 1 from Table 4.1 (extracting bits in odd positions)

\begin{tabular}{|l|l|l|l|l|}
\hline Index & Sub-Prefix & Len & Port Indicator & Forwarding Information \\
\hline 1 & $011001^{*}$ & 6 & 010 & $2(2)$ \\
2 & $0110011^{*}$ & 7 & 001 & $3(4)$ \\
3 & $011011^{*}$ & 6 & 010 & $2(7)$ \\
4 & $0110110^{*}$ & 7 & 010 & $2(8)$ \\
5 & $10010^{*}$ & 5 & 010 & $2(1)$ \\
6 & $100100^{*}$ & 6 & 100 & $1(3)$ \\
7 & $10010011^{*}$ & 8 & 100 & $1(4)$ \\
8 & $100110^{*}$ & 6 & 100 & $1(2)$ \\
9 & $1001010^{*}$ & 7 & 001 & $3(2)$ \\
10 & $100110^{*}$ & 6 & 010 & $2(3)$ \\
11 & $1001101^{*}$ & 7 & 100 & $1(1)$ \\
12 & $10011011^{*}$ & 8 & 001 & $3(1)$ \\
13 & $1011101^{*}$ & 7 & 011 & $2(4), 3(3)$ \\
14 & $101111^{*}$ & 6 & 010 & $2(5)$ \\
15 & $11110^{*}$ & 5 & 010 & $2(6)$ \\
\hline
\end{tabular}


Table 4.3: Sub-Forwarding Table 2 from Table 4.1 (extracting bits in even positions)

\begin{tabular}{|l|l|l|l|l|}
\hline Index & Sub-Prefix & Len & Port Indicator & Forwarding Information \\
\hline 1 & $000001^{*}$ & 6 & 010 & $2(5)$ \\
2 & $000010^{*}$ & 6 & 001 & $3(3)$ \\
3 & $0000101^{*}$ & 7 & 010 & $2(4)$ \\
4 & $10100^{*}$ & 5 & 010 & $2(3)$ \\
5 & $1010010^{*}$ & 7 & 100 & $1(1)$ \\
6 & $10100100^{*}$ & 8 & 001 & $3(1)$ \\
7 & $10101^{*}$ & 5 & 110 & $2(1), 1(2), 1(3)$ \\
8 & $101011^{*}$ & 6 & 001 & $3(2)$ \\
9 & $10111^{*}$ & 5 & 010 & $2(6)$ \\
10 & $11000^{*}$ & 5 & 010 & $2(7)$ \\
11 & $110001^{*}$ & 6 & 010 & $2(2)$ \\
12 & $1100010^{*}$ & 7 & 001 & $3(4)$ \\
13 & $110010^{*}$ & 6 & 010 & $2(8)$ \\
14 & $11110001^{*}$ & 8 & 100 & $1(4)$ \\
\hline
\end{tabular}


ing index. In general, the forwarding information of each sub-entry in a sub-table may consist of multiple forwarding units. For example, the sub-prefix of the $7^{\text {th }}$ entry in Table 4.3 is $10101^{*}$, which collects the information of the original prefixes whose bits in the even positions are $10101^{*}$. Its Forwarding Information is composed of three forwarding units, 2(1), 1(2), and 1(3).

Based on today's technology, a 20-bit long sequence is enough to represent a forwarding unit. Let $n$ bits beginning from the left-most be used to represent the port number, $a$. Let the remainder ( $20-\mathrm{n}$ bits) be used to represent the index number, $b$. Usually, a core router has no more than 128 output ports, and $n$ is no more than 7 bits. Therefore, at least 13 bits are reserved for the index number (allowing up to 8192 entries that could be forwarded to the same port).

In a sub-table, Let an $N$-bit Port Indicator be attached to each sub-entry, where $N$ is the number of all distinct ports. The bit at the $t^{t h}$ position beginning from the leftmost in the Port Indicator is set to 1 if and only if the $i^{\text {th }}$ port occurs in its associated forwarding information, otherwise it is set to 0 . For example, the forwarding information of the first sub-entry in Table 4.2 is 2(2), and there are 3 ports in total, so that its Port Indicator is set to 010 . Usually the width of a Port Indicator is no more than 128 bits. The total storage cost for the extra information is shown in the last row of Table 4.4.

What is the merit of the CES? We investigate it from the following two main aspects.

1. Since a forwarding table is decomposed into two sub-tables, one lookup is transformed into a pair of parallel sub-lookups. Can CES allow the pair of sub-lookups in balance, both in memory access time and in storage requirement?

2. Some matching sub-prefixes are got after the pair of parallel sub-lookups. In order 
to achieve the BMP, it is required to compare the information of any reasonable pair of matching sub-prefixes from distinct sub-tables. Can CES result in heavy comparison loads, which in turn can be the bottleneck of a complete $\mathbb{P}$ address lookup?

Firstly, CES makes the entries of the pair of sub-tables well distributed.

In comparing two bit patterns, the Hamming distance is the count of bits different in the two patterns. We will give a new definition to determine the distance between two prefixes, which is similar to the Hamming distance.

Definition 4.1 (Pseudo-Hamming Distance (PHD)). Let $a$ and $b$ be two prefixes in $a$ table. $|a|$ and $|b|$ are used to represent their lengths. Let $M=\min (|a|,|b|)$. We define the PHD between two prefixes as:

$$
P H D(a, b)=\sum_{i=1}^{M}(L-i+1)\left|a_{i}-b_{i}\right|,
$$

where $a_{i}$ and $b_{i}$ are the bits in the $i^{t h}$ position, from the left-most, of $a$ and $b$, and $L$ is the maximum length of the sequences (In $\operatorname{IPv} 4, L$ is $32 . L$ is 16 in the sub-forwarding tables).

From this definition, we can see that the difference in the higher-order (left) bit contributes more than the difference in the lower-order (right) bit. Let $M P H D$ be the mean of the PHDs among all the pairs of distinct prefixes in the table. PHD is affected by both the number of bits that are not identical, and their positions. For example, assuming $a, b$ and $c$ are $011001^{*}, 0110010^{*}$, and $10010011^{*}$ respectively. Let $L$ be $16 . P H D(a, b)=0$, $P H D(a, c)=79$, and $P H D(b, c)=79$.

Definition 4.2 (Parent). Let $P 1$ and $P 2$ be two prefixes. We say prefix $P 1$ is the parent of prefix $P 2$, if both of the following conditions hold: 
1. $|P 1|<|P 2|$

2. $\forall$ the $i^{\text {th }}$ bit in $P 1$ and $P 2$, there must exist $P 1_{i}=P 2_{i}$, where $1 \leq i \leq|P 1|$.

Lemma 1. If $P 1$ and $P 2$ are two prefixes, such that one is the parent of the other, then PHD $(P 1, P 2)$ equals to zero.

In the previous example, $a$ is the parent of $b$, and $P H D(a, b)=0$. The value of MPHD is a representation for the distribution of entries in a table. If the value of MPHD is big, it implies that in the trie of a forwarding table, nodes are spread widely rather than located in some specific deep branches. It allows for a faster search if $M P H D$ is big.

Let prefix $a$ be divided into $a^{\prime}$ and $a^{\prime \prime}$, and $b$ be divided into $b^{\prime}$ and $b^{\prime \prime}$ by CES. We can prove that:

$$
\begin{aligned}
& 0 \leq 2\left(P H D\left(a^{\prime}, b^{\prime}\right)+P H D\left(a^{\prime \prime}, b^{\prime \prime}\right)\right)-P H D(a, b) \leq\left\lfloor\frac{M}{2}\right\rfloor \\
& \left|P H D\left(a^{\prime}, b^{\prime}\right)-P H D\left(a^{\prime \prime}, b^{\prime \prime}\right)\right| \leq \frac{M}{4}\left(\frac{M}{2}+L+3\right)
\end{aligned}
$$

It demonstrates that CES will not result in big difference in MPHDs between the pair of sub-tables, when MPHD of the original forwarding table is fixed, and the bits of 0 s and 1s distribute randomly. The distribution of the sub-entries in each sub-table is consistent with the distribution of the entries in the original forwarding table. It indicates that CES leads to a balanced distribution of entries in the two sub-tables.

Secondly, CES also balances the sub-prefix lengths in the pair of sub-tables.

Definition 4.3 (Mean Prefix Length (MPL)). : Let MPL in a sub-table be expressed by $\frac{1}{N} \sum_{i=1}^{S} n_{I F O}^{i} L e n_{i}$, where $N$ is the number of entries in the original forwarding table, $S$ is the number of the sub-entries in this sub-table, $n_{I F O}^{i}$ is the number of forwarding units attached to the $i^{t h}$ sub-entry, and $L e n_{i}$ is the length of it. 
Let $a$ be an original prefix in a forwarding table. It is converted into two sub-prefixes, named $a^{\prime}$ and $a^{\prime \prime}$, by using CES. The difference between the lengths of $d^{\prime}$ and $a^{\prime \prime}$ satisfies the inequality: $0 \leq\left|a^{\prime}\right|-\left|a^{\prime \prime}\right| \leq 1$. In fact, the difference between the $M P L$ s of the pair of sub-tables based on $C E S$ is due to the number of the original prefixes, whose lengths are odd values. It is effective to minimize the difference between the lookup time taken in the pair of sub-tables, in that the sub-prefix lengths in both sub-tables are almost the same. $C E S$ is an efficient way to allow the memory access times in the pair of sub-tables to keep in pace. With respect to the entries in Table 4.1, MPLs of the pair of sub-tables, Table 4.2 and Table 4.3 , are 6.5 and 6.06 , respectively.

Thirdly, CES makes the forwarding units well distributed in each sub-table.

Definition 4.4. (I) Basic Load of Forwarding Information (BLFI) of the $i^{\text {th }}$ subentry in each sub-table is defined as the number of forwarding units in the $i^{\text {th }}$ subentry.

(2) Mean Load of Forwarding Information (MLFI) of the subentries in a subtable is defined by $\frac{1}{S} \sum_{i=1}^{S} B L F I_{i}$.

(3) Standard Deviation of Forwarding Information (SDFI) of the subentries in each sub-table is defined by $\sqrt{\frac{1}{S} \sum_{i=1}^{S}\left(B L F I_{i}-M L F I\right)^{2}}$.

These metrics are significant when analyzing $C E S$ 's performance. Small values are desirable. The performance of the comparison delay between the matching sub-prefixes in the second phase (comparison set) relies on the these three factors. Small difference on $M L F I \mathrm{~s}$ (or SDFIs) between the pair of sub-tables is also expected. Regarding the given entries in Table 4.1, $M L F I_{1}=1.067, M L F I_{2}=1.143, S D F I_{1}=0.25$, and $S D F I_{1}=0.51$ 
in Table 4.2 and Table 4.3.

Fourthly, $C E S$ balances the comparison cost.

Definition 4.5 (Comparison Cost Factor (CCF)). CCF is used to judge whether the comparison load (the number of the compared forwarding information pairs) of those matching sub-prefixes from the two sub-tables is heavy or not. CCF is a statistical value from experiments, by counting the real compared pairs.

Actually, it is not required to compare every pair of matching sub-prefixes. If $a^{\prime}$ and $a^{\prime \prime}$ are the pair of sub-prefixes that are decomposed from $a$ using $C E S$, then they should satisfy the following conditions:

- $\left|a^{\prime \prime}\right|$ should be equal to either $\left|a^{\prime}\right|$ or $\left|a^{\prime}\right|-1$.

- In the corresponding attached Port Indicator, there must exist at least one bit $i \leq N$, such that $P I V_{i}^{1}=P I V_{i}^{2}=1\left(P I V^{1}\right.$ and $P I V^{2}$ stand for the Port Indicators attached to $d^{\prime}$ and $a^{\prime \prime}$, respectively).

Only if the matching sub-prefixes $a^{\prime}$ and $a^{\prime \prime}$, which are got from distinct sub-tables, meet the demands above is the further forwarding information comparison needed. $C C F$ is a parameter to observe the number of the sub-prefixes pairs that need real forwarding information comparison operated.

$C C F$ has its upper bound: Let $\operatorname{MinNum}=\min \left(N u m_{1}, N u m_{2}\right)$, where $N u m_{1}$ and $N u m_{2}$ are the numbers of matching sub-prefixes in sub-table 1 and sub-table 2, respectively. Then, $C C F \leq 2 \times M i n N u m$.

We downloaded IPv4 routing tables from http://www.merit.edu/ipma/routing able for experiment, which is not available right now. We have 4 forwarding tables, Mae-East, 
Table 4.4: Performance of the Pair Sub-tables by Using CES

\begin{tabular}{|l|l|l|l|l|l|l|l|l|}
\hline & \multicolumn{2}{|c|}{ Mae-east } & \multicolumn{2}{c|}{ Mae-west } & \multicolumn{2}{c|}{ Aads } & \multicolumn{2}{c|}{ Paix } \\
\hline & \multicolumn{2}{|c|}{47206} & \multicolumn{2}{c|}{77002} & \multicolumn{2}{c|}{63980} & \multicolumn{2}{c|}{22116} \\
\hline & $\begin{array}{l}\text { Sub- } \\
\text { table1 }\end{array}$ & $\begin{array}{l}\text { Sub- } \\
\text { table2 }\end{array}$ & $\begin{array}{l}\text { Sub- } \\
\text { table1 }\end{array}$ & $\begin{array}{l}\text { Sub- } \\
\text { table2 }\end{array}$ & $\begin{array}{l}\text { Sub- } \\
\text { table1 }\end{array}$ & $\begin{array}{l}\text { Sub- } \\
\text { table2 }\end{array}$ & $\begin{array}{l}\text { Sub- } \\
\text { table1 }\end{array}$ & $\begin{array}{l}\text { Sub- } \\
\text { table2 }\end{array}$ \\
\hline sub-entries & 4026 & 5341 & 5703 & 6989 & 5689 & 6735 & 4077 & 4704 \\
\hline MPHD & 55.22 & 56.56 & 56.47 & 57.84 & 56.80 & 57.45 & 54.59 & 55.67 \\
\hline MPL & 11.18 & 11.18 & 11.22 & 11.22 & 11.35 & 11.35 & 11.15 & 11.15 \\
\hline Max(BLFI) & 93 & 86 & 100 & 78 & 110 & 89 & 40 & 28 \\
\hline MLFI & 11.73 & 8.84 & 13.05 & 11.02 & 11.25 & 9.50 & 5.42 & 4.70 \\
\hline SDFI & 13.41 & 9.71 & 15.49 & 12.57 & 14.28 & 10.84 & 5.35 & 4.23 \\
\hline CCF & \multicolumn{2}{|c|}{8} & \multicolumn{2}{|c|}{8} & & 8 & 7 \\
\hline $\begin{array}{l}\text { Storage Cost } \\
\text { (in KByte) }\end{array}$ & 186.06 & 209.15 & 270.81 & 241.95 & 245.14 & 261.44 & 117.65 & 127.48 \\
\hline
\end{tabular}

Mae-West, Aads and Paix, from different NAPs. Table 4.4 gives us the performance result of the pair of sub-tables after using $C E S$. In order to provide the evidence that $C E S$ is an outstanding splitting rule, we introduce another contrasting direct splitting rule, in which the higher 16 bits are extracted to form sub-table 1 and the lower 16 bits are extracted to form sub-table 2. Table 4.5 gives us the result of the pair of sub-tables by using this direct splitting rule. After comparing the results from those two tables, we can see that the CES has better performance than the second one. 
Table 4.5: Performance of the Pair Sub-tables by Such a Splitting Rule: extracting the higher 16 bits to form sub-table 1 and extracting the lower 16 bits to form sub-table 2

\begin{tabular}{|l|l|l|l|l|l|l|l|l|}
\hline & \multicolumn{2}{|c|}{ Mae-east } & \multicolumn{2}{c|}{ Mae-west } & \multicolumn{2}{c|}{ Aads } & \multicolumn{2}{c|}{ Paix } \\
\hline \multirow{2}{*}{ Entries } & \multicolumn{2}{|c|}{47206} & \multicolumn{2}{c|}{77002} & \multicolumn{2}{c|}{63980} & \multicolumn{2}{c|}{22116} \\
\hline & $\begin{array}{l}\text { Sub- } \\
\text { table1 }\end{array}$ & $\begin{array}{l}\text { Sub- } \\
\text { table2 }\end{array}$ & $\begin{array}{l}\text { Sub- } \\
\text { table1 }\end{array}$ & $\begin{array}{l}\text { Sub- } \\
\text { table2 } 2\end{array}$ & $\begin{array}{l}\text { Sub- } \\
\text { table1 }\end{array}$ & $\begin{array}{l}\text { Sub- } \\
\text { table2 }\end{array}$ & $\begin{array}{l}\text { Sub- } \\
\text { table1 }\end{array}$ & $\begin{array}{l}\text { Sub- } \\
\text { table2 }\end{array}$ \\
\hline sub-entries & 6939 & 1349 & 10794 & 1692 & 8314 & 3540 & 1328 & 4704 \\
\hline MPHD & 59.85 & 43.24 & 23.32 & 51.63 & 61.54 & 49.03 & 59.68 & 48.85 \\
\hline MPL & 16.00 & 6.47 & 16.00 & 4.79 & 16.00 & 9.94 & 16.00 & 5.03 \\
\hline Max(BLFI) & 280 & 2735 & 253 & 5939 & 465 & 3385 & 128 & 1406 \\
\hline MLFI & 6.80 & 34.99 & 7.13 & 45.50 & 7.69 & 18.07 & 4.87 & 17.86 \\
\hline SDFI & 13.63 & 93.62 & 15.33 & 164.45 & 16.65 & 73.38 & 7.98 & 49.95 \\
\hline
\end{tabular}

\subsection{Comparison Set}

To complete a full step of comparing a pair of matching sub-prefixes, two steps are needed. The first step is to judge whether the further comparison is required, which has already been discussed in the previous section. The second step, which is the operation to compare the forwarding information, will be discussed in this section. During this step, the final common matching prefix and its output port can be determined. It can be implemented in an ASIC.

Let an incoming destination address $a$ be decomposed into $a^{\prime}$ and $a^{\prime \prime}$ by using CES. 
Assuming that $P 1_{i}$ is a matching sub-prefix of $a^{\prime}$ in sub-table 1 and $P 2_{j}$ is the matching sub-prefixes of $a^{\prime \prime}$ in sub-table 2. $P 1_{i}$ is attached by forwarding information $\operatorname{Info} 1_{i}$ and $P 2_{j}$ is attached by forwarding information $\operatorname{Info} 2_{j} . P 1_{i}$ and $P 2_{j}$ are called a matched pair of sub-prefixes, if there exists a common forwarding unit in $I n f o 1_{i}$ and $I n f o 2_{j}$. In the comparison process, each forwarding unit in $I n f o 1_{i}$ must be compared with all the forwarding units in $I n f o 2_{j}$. Let $I n f o 1_{i}$ and $I n f o 2_{j}$ contain $M 1$ and $M 2$ forwarding units, respectively. In order to compare a pair of such sub-prefixes, the total pairs of the compared forwarding units needed are the product of $M 1$ and $M 2$.

If $P 1_{i}$ and $P 2_{j}$ are a matched pair of sub-prefixes, it indicates that they are the right pair of sub-prefixes that are decomposed from an original prefix in a forwarding table.

Lemma 2. In the comparison between Info $1_{i}$ and Info $2_{j}$, there exists at most one exact match among all pairs of compared forwarding units.

Proof: Info $1_{i, k}$ and $I n f o 1_{i, l}$ are two of the forwarding units contained in Info $_{i}$. $I n f o 1_{j, m}$ and $I n f o 1_{j, n}$ are two of the forwarding units contained in $I n f o 1_{j}$. Assume

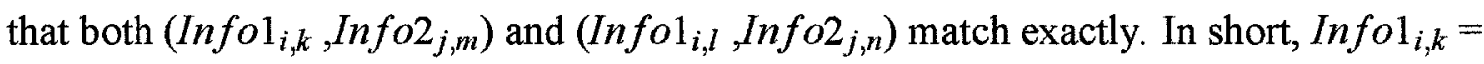
Info $2_{j, m}$, and $I n f o 1_{i, l}=\operatorname{Info2_{j,n}}$. It indicates that in the original forwarding table, there exist two entries, whose prefixes are identical, but will be forwarded to distinct output ports. It is impossible for unicast. Hence, as a consequence, the assumption is not right. Therefore, there exists at most one exact match in all pairs of comparison units.

Since each forwarding unit is 20-bit long, based on todays technology, VLSI feature size of $\lambda=0.13 \mu \mathrm{m}$, it is possible to input 5 forwarding units from each matching subprefix for comparison simultaneously, which is restricted by the maximum number of gates and pins per-chip. It allows us to process 25 parallel comparison units. Therefore, 
all the comparison units are processed in a sequencing order by at most 25 pairs to the end or until one pair of matching forwarding units occur. The time cost of every 25 parallel comparisons is $250 \mathrm{ps}$, which is got from our experiments. Table 4.6 tells us the delay in comparing a pair of matching sub-prefixes. It is clear to see that the delay increases when the size of the forwarding table grows. Actually, the real cost is smaller than this, since the comparison stops when there exists an exact match. The delay in most cases is satisfactory, although the delay in the worst case is not good enough.

Table 4.6: Delay for the Comparison between One Pair of Sub-Prefixes

\begin{tabular}{|l|l|l|l|l|}
\hline & Entries & Average Delay (ns) & $\begin{array}{l}\text { Delay (80\%of } \\
\text { comparisons)(ns) }\end{array}$ & Worst Case (ns) \\
\hline Mae-east & 47206 & 1.39 & $<8.58$ & 36.9 \\
\hline Mae-west & 80000 & 3.04 & $<12.87$ & 56.5 \\
\hline Aads & 63980 & 2.74 & $<9.06$ & 54.5 \\
\hline Paix & 22116 & 0.40 & $<1.55$ & 5.26 \\
\hline
\end{tabular}

\subsection{Performance Description}

Figure 4.2 describes a general picture of how the CES-based address lookup system operates. The initial forwarding table is decomposed into a pair of extended sub-tables based on CES. Searches in both sub-tables are independent, since searches for the matching sub-prefix in one sub-table does not affect the searches in other one. The pair of 
sub-lookups can be processed simultaneously. In each sub-table, once a matching subprefix occurs, the attached information will be sent to the comparison set while the further sub-lookup for another matching sub-prefix continues. The pair of the sub-lookups and the comparison are allowed to be processed simultaneously. Therefore, the proposed CES provides a way to migrate the general IP address lookup problem over a single sequential process to multiple parallel small processes, which is helpful for the next generation network to improve the performance of the packet processing. In the remainder of this section, two basic structures of the sub-tables will be described, which are the variants of the index table and the binary trie. Furthermore, their associated search strategies and performance will be discussed.

\subsubsection{CES + Index tables}

After using CES decomposition method, the maximum length of the sub-prefixes in a sub-table is halfed. The row length of the index array of each sub-table is $2^{16}$, while that of the initial index array is $2^{32}$ for IPv4. The summation of the lengths of the pair of arrays is $2^{17}$, which is reduced to be one out of $2^{15}$ of an original IPv4 index table. A pair of index tables are applicable for practical use.

\section{Architecture}

When deploying the CES + Index tables method, each sub-table is composed of a major index table and a forwarding information list. Each entry in the major index table has the data structure shown in Figure 4.3. In each entry, Length, a 4-bit long string, 


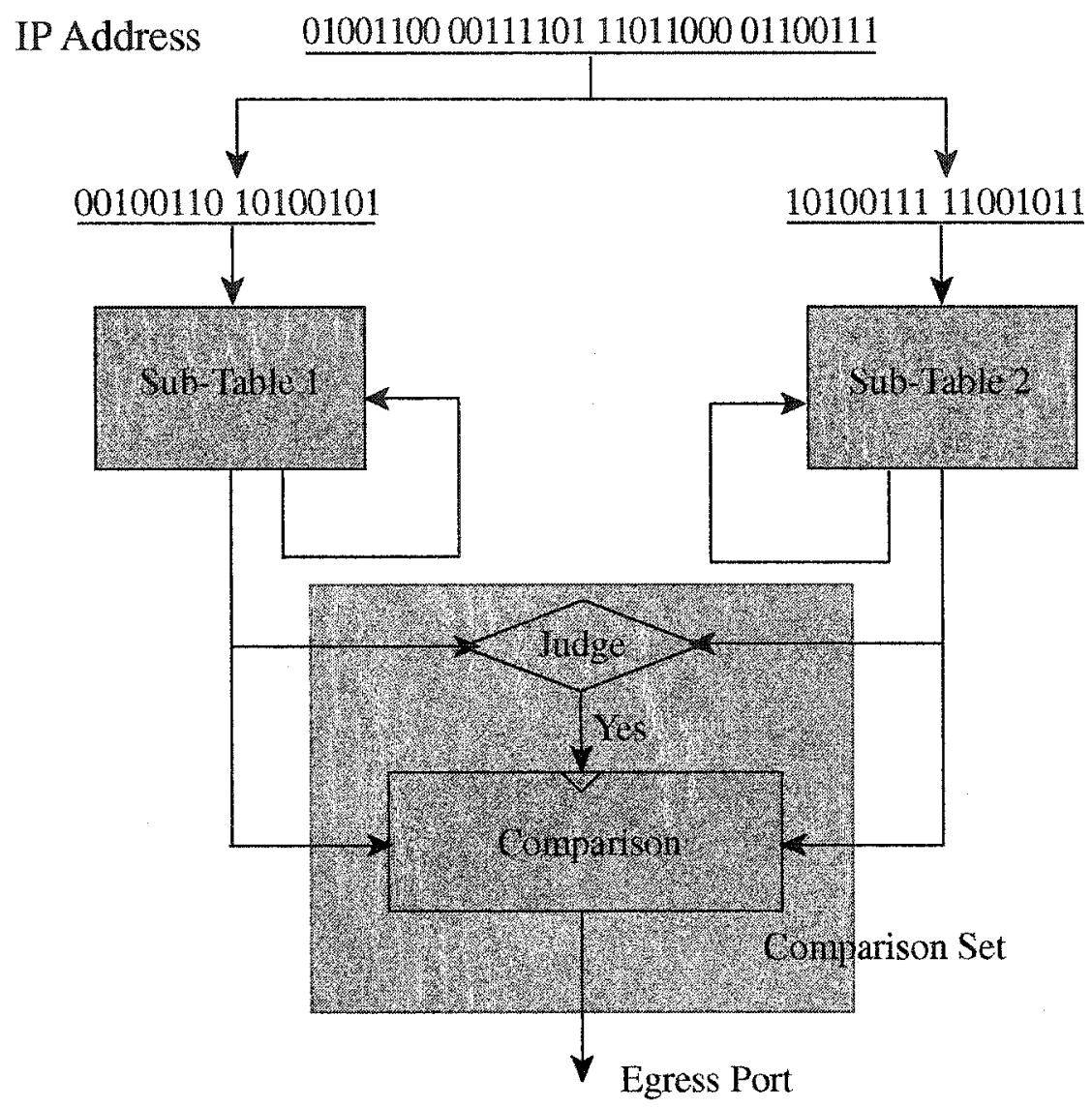

Figure 4.2: Architecture of the Algorithm

stands for the length of the sub-prefix. Port Indicator is a 128-bit long string. Each bit in it corresponds to one port, and it is set to 1 if the corresponding port occurs in the forwarding information set, otherwise it is set to 0 . The 16-bit long pParent points to the beginning index of its most specific parent, which will be discussed below. In Figure 4.4, all subprefix entries have to be expanded to 16 -bit long. For instance, 1001101* is expanded to the interval $[1001101000000000,1001101111111111]$, which can be expressed by the decimal interval [39424, 39935]. 10011011* is expanded to the interval [39680, 39935], 


\begin{tabular}{|l|l|l|l|}
\hline Length [4] & Port Indicator [128] & pParent [16] & pinformation [16] \\
\hline
\end{tabular}

Figure 4.3: Structure of an Entry in the Sub Index Table

and $100110^{*}$ is expanded to the interval [38912, 39935]. Both $1001101^{*}$ and $100110^{*}$ are the parents of $10011011^{*}$ in sub-table 1 . Since $1001101^{*}$ is longer than $100110^{*}$, we call the former one the most specific parent of $10011011^{*}$ and all the pParents of the entries from index 39680 to index 39935 (10011011*) point to index 39424, which is the beginning index of $1001101^{*}$. If a sub-prefix does not have any parent, then its pParent will be set NULL. pInformation is a pointer to its forwarding information. Information list is a list to store the forwarding information, each entry of which corresponds to one sub-prefix.

Each major index table consumes $1.28 \mathrm{Mbytes}$, which is a stable memory cost. However the additional information list for forwarding information varies with the forwarding table. Fortunately, the memory cost of the information list is very small compared to the major index table, which was shown in Table 4.4. The total memory consumption is about 3Mbytes.

This architecture is not scalable to IPv6, for the size of the major index table is $2^{64}$, which is still hard to be achieved by current technology.

\section{Search Strategy - First Match}

When a search starts, the first matching sub-prefix reached in each major index table is the longest matching sub-prefix. For example, when looking for the matching sub-prefix of 1001101111000000 (39872) in the index table in Figure 4.4, 10011011* will be got at 
the first cycle, $1001101 *$ will be got at the second cycle and $100110^{*}$ will be got at the last cycle. Not only is the forwarding information of each matching sub-prefix sent to the comparison set, but also its own most specific parent row is pointed, and another pair of forwarding information will be ready for comparison. At each time, since the entry in sub-index table reached is directed by the pointer recorded in the previous reached entry, the length of the sub-prefix got in this cycle is shorter than the one that got in the previous cycles. Therefore once there is an exact match in the comparison set, the search terminates. The average number of comparisons in our experiments were 1.272 , so the average of the total delay in comparison is no more than $8 \mathrm{~ns}$ (if the total of entries is no more than $80 \mathrm{~K}$ ).

\begin{tabular}{|c|c|c|c|c|c|c|}
\hline Index & sub-prefix & Length & Port Indicator & pParent & plnformation & \\
\hline 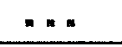 & $\ldots$ & $m=$ & $=\ldots$ & $m \infty$ & $m$ & \\
\hline \multirow[t]{3}{*}{38912} & $100110 *$ & 6 & 010 & NULL & $\mathrm{L}$ & \\
\hline & & 6 & 010 & NULL & $\mathbf{L}$ & \\
\hline & & 6 & 010 & NULL & $L$ & $=-$ \\
\hline \multirow[t]{2}{*}{39424} & $1001101 *$ & 7 & 100 & 38912 & M & $2(3)$ \\
\hline & & 7 & 100 & 38912 & M & $1(1)$ \\
\hline \multirow[t]{2}{*}{39680} & $10011011^{*}$ & 8 & 001 & 39424 & $\mathrm{~N}$ & $3(1)$ \\
\hline & & 8 & 001 & 39424 & $\mathbf{N}$ & $m \ldots$ \\
\hline 39935 & $10011011^{*}$ & 8 & 001 & 39424 & $\mathrm{~N}$ & $2(4) 3(3)$ \\
\hline$m$ & $\ldots$ & $\cdots$ & $\therefore=$ & $m=m$ & $\ldots=$ & 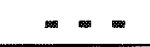 \\
\hline \multirow[t]{2}{*}{47616} & $1011101 *$ & 7 & 011 & NULL & $\mathbf{R}$ & \multirow{4}{*}{ mation I } \\
\hline & & 7 & 011 & NULL & $\mathrm{R}$ & \\
\hline 48127 & $1011101 *$ & 7 & 011 & NULL & $\mathrm{R}$ & \\
\hline$\ldots$ & $\ldots$ & $\therefore=$ & $\therefore \approx$ & $\therefore=$ & $\infty$ & \\
\hline
\end{tabular}

Major Index Table

Figure 4.4: The Structure of CES + Index tables 


\subsubsection{CES + Binary Tries}

The binary trie is a basic structure in IP lookups. A forwarding table is decomposed into a pair of half-level (16-level) sub-tables. The storage requirement for a pair of 16level binary tries is much smaller than one 32 -level binary trie. Therefore, a $\left(2^{33}-1\right)$-node original binary trie is converted to a pair of $2\left(2^{17}-1\right)$-node sub-binary tries. In short, the nodes in the original binary trie are shrunk to one of $2^{15}$.

\section{Architecture}

Table 4.7: Storage Cost Comparison (CES+Binary Trie VS Binary Trie) (in Byte)

\begin{tabular}{|l|l|l|l|l|}
\hline Storage Cost & Mae-east & Mae-west & Aads & Paix \\
\hline Sub-Table 1 & $215.5 \mathrm{~K}$ & $310.2 \mathrm{~K}$ & $285.6 \mathrm{~K}$ & $147.8 \mathrm{~K}$ \\
\hline Sub-Table 2 & $247.2 \mathrm{~K}$ & $288.9 \mathrm{~K}$ & $308.3 \mathrm{~K}$ & $161.6 \mathrm{~K}$ \\
\hline Original Table & $1295.3 \mathrm{~K}$ & $2003.8 \mathrm{~K}$ & $1657.8 \mathrm{~K}$ & $718.8 \mathrm{~K}$ \\
\hline
\end{tabular}

The information contained in each node is: \{pLefiChild[32], pRightChild[32], Len[4], Port Indicator [128], pInformation [32] \}. pLeftChild and pRightChild are the pointers to its left child node and right child node. Len records the number of the nodes traversed from the root. pInformation is a pointer to its forwarding information set. The total storage requirement is a combination of the size of the pair of sub-binary tries and their forwarding information sets. Table 4.7 compares the storage requirement between using CES-based binary tries and original binary trie. It shows that the storage consumption of 
CES-based binary trie is more efficient, which is less than half size of the original binary trie.

\section{Search Strategy - Last Match}

When a search starts, the first matching sub-prefix we reach in each sub-table is the shortest one. It is required to compare all possible pairs of forwarding information, and meanwhile, traversing the sub-tries continues until they are exhaustive. The output port contained in the last exact matching forwarding unit is the final egress port of this IP packet. The total average delay in comparison is no more than $25 \mathrm{~ns}$, since the CCF is less than 8 (if the total entries are not more than $80 \mathrm{~K}$ ).

There is a pipeline benefit, no matter which architecture we use: the comparison set operates when both sub-lookups are being processed for the next pair of sub-prefixes. From the experimental results, we can see that the comparison set is fast enough, so that it can not be a bottleneck, if the forwarding table is not too big.

We can also make use of variants of the binary trie, such as the multibit trie and LC trie to reduce the length of each sub-trie, so that the performance of either time complexity or storage complexity of the algorithm can be further improved. But at each node, more information has to be recorded for possible backtrack.

\subsubsection{Possible Variant Discussion}

In this chapter, a CES method is created to decompose a forwarding table into a pair of sub-tables. A single lookup procedure is converted into parallel small lookups in a pair 
of sub-tables and a comparison set. We investigate that whether we can decompose one forwarding table into more sub-tables to allow more parallel processes to be operated.

A prefix is decomposed into $m$ sub-prefixes directly by using the variant of CES, where $m>2$. Beginning from the left-most bit, the $1^{s t},(m+1)^{t h},(2 m+1)^{t h}, \ldots,(i m+$ $1)^{t h}, \ldots$ bits are extracted to form sub-prefix 1 . Then the $2^{\text {nd }},(m+2)^{t h},(2 m+2)^{t h}, \ldots$, $(i m+2)^{t h}, \ldots$ bits are extracted to form sub-prefix 2. ... . The $m^{t h},(2 m)^{t h},(3 m)^{t h}, \ldots$, $((i+1) m)^{t h}, \ldots$ bits are extracted to form sub-prefix $m$. A forwarding table is transformed into $m$ small extended sub-tables. When a matching sub-prefix occurs in each sub-lookup, one comparison among those $m$ matching sub-prefixes from distinct sub-tables will be operated at once. Both the sub-lookup time and the storage cost of the sub-tables are further reduced.

Unfortunately, since the number of the matching sub-prefixes in each sub-table and the number of the attached forwarding units to each sub-entry may increase, it will result in heavy load in the comparison set. One comparison among $m$ matching sub-prefixes may be composed of a few of phases. For example, let $m$ be 4 . In the first phase, the matching sub-prefixes from sub-table 1 and sub-table 3 are grouped, so do the matching sub-prefixes from sub-table 2 and sub-table 4 . The comparison between the pair of subprefixes in each group performs individually. The draft common forwarding units from each comparison can be determined. In the subsequent phase, the common forwarding units from both comparison set in the earlier phase are compared again to find the final matching forwarding unit.

The performance of a complete lookup relies on the combination performance of sublookups and the comparison set. If the search in sub-tables is slower than the speed of the comparison, it needs further decomposition in each sub-tables. It is called the best search 
structure of a given forwarding table, only when both speeds are comparable.

\subsection{Summary}

We proposed a new methodology and architecture for IP address lookup. In this chapter, one sequential IP address lookup process is migrated to parallel multiple sub-lookup processes. Our approach advocates decomposing a forwarding table into a pair of subforwarding tables using CES. Comparison is only needed for the reasonable matching sub-prefixes of the pair of sub-tables. The pair of sub-lookups processes and one comparison process can operate in parallel, which provides a new way to speed up the average search time efficiently to handle $\mathrm{OC}-192$ line rates $(10 \mathrm{~Gb} / \mathrm{s})$. Unfortunately, with the growth of the forwarding table, the number of the forwarding units attached to a subprefix increases. If both comparing sub-prefixes carry hundreds of forwarding units, the comparison delay will affect the performance of the whole system. CES cannot improve the performance much in worst cases, but can make a big improvement for the average search time. There is still great potential for the further improvement of the comparison set, when the load is heavy. 


\section{Chapter 5}

\section{Packet Classification Using Bits}

\section{Characteristics}

In this chapter, we will describe a new algorithm for packet classification making use of bits distribution features. It is a high level classification method. It always takes the bits from every dimension into account, instead of constraining the search process in some of the dimensions at every stage. The experiments provide the evidence that it has outstanding performance even for large edge classifiers. It is also scalable to IPv6.

\subsection{Introduction}

All the rules in a multi-dimensional classifier can be regarded as a list of one-dimensional mask-based binary strings. The lengths of all the strings are equal. In these strings, those 
bits defined as 0 or 1 are what we are interested in, while others defined as * (not masked) are what we are not interested in. The packet classification problem is transformed to a longer one-dimensional matching problem. For example, a two-dimensional packet classification in IPv4 (IP Source Prefix, and IP Destination Prefix) can be considered as a one-dimensional 64-bit wide table lookup. The main advantage of this concept is that the one-dimensional search algorithm is much simpler.

In proposing the new algorithm in this thesis, firstly, we analyze the bits characteristics possessed by each rule and then create a hash function to represent the distribution of the bits in each rule. Therefore, all the rules can be mapped into a set of hash buckets based on their distinct distribution features. This is a high-level approach to eliminate as many rules as possible at the first step. The relationship among the rules colliding in one bucket is "loose" in that they do not have the intuitive regularity, hence they do not have the same prefix or they are not confined to certain intervals in some specific dimensions. This kind of hash method can overcome the problem of unbalanced distribution (filters are only distributed in some particular small ranges) in some dimensions. It even has better performance when it operates for edge routers, compared with other hash functions.

Secondly, this proposed algorithm also tries to build a global optimal search trie in each bucket by inspecting the most significant bit positions. The search path is directed by the bits chosen from all the dimensions at each step, other than being confined to the bits from one or some of the multi-dimensions. The goal of the search trie is to reduce the search range as much as possible at each stage while minimizing the number of examined bit positions. Branching terminates when the number of the remaining rules in a node is smaller than the pre-defined maximum number.

Thirdly, in the leaves of the search trie, a matching process to the rules is needed 
until the highest priority matching is found. Hardware-based search can speed up the procedure.

Our algorithm is unique in the sense that each step always tries to reach a high-level optimal solution, by examining all the possible bits over all, other than concentrating on some consecutive bits in a specific dimension. It provides a good average case performance and consumes a reasonable amount of storage. It performs well for large classifiers or edge classifiers. We also examine different issues relating to the practical implementation of our approach.

\subsection{The Structure of the Proposed Algorithm}

The proposed algorithm is composed of three layers as shown in Figure 5.1. Firstly, a heuristic hash function is defined to assign all the rules in a classifier to a set of buckets according to their bits distribution features. Then in each bucket, a decision tree is built, which is based on inspecting certain specific bits position. Finally, all the leaf nodes store a small number of rules, and a linear search among these rules yields the desired matching.

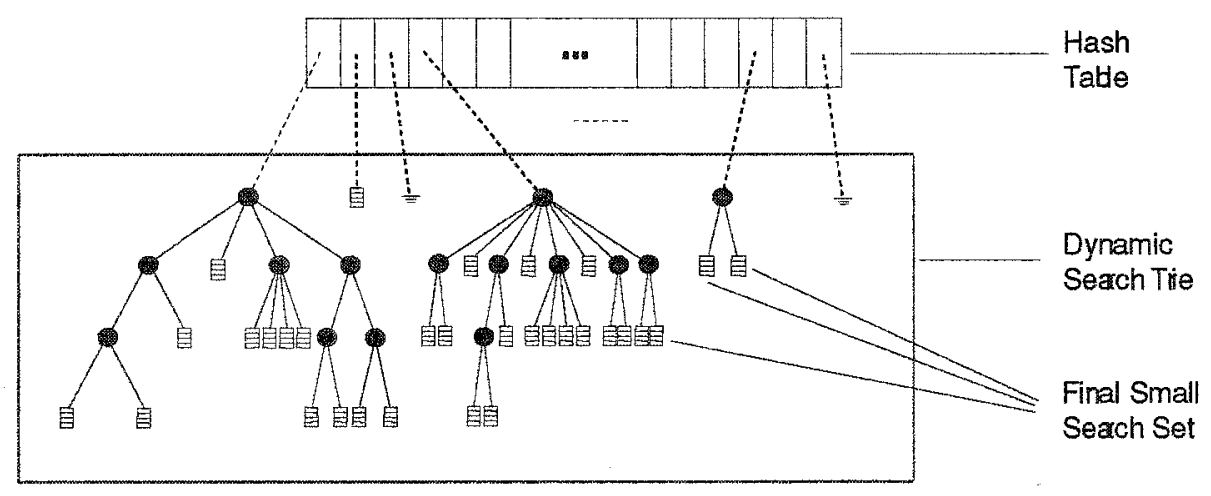

Figure 5.1: The Structure of the Proposed Algorithm 


\subsubsection{Hash Table}

In this subsection, first, we will introduce a novel hash function and explain its principles. Then, we will describe the data structure of the resulting (hashing) table built according to the proposed hash function.

\section{Definition of the hash function}

This proposed hash function is based on the bits distribution. In other words, it can reflect how 1's (or 0's) spread in the sequences. Then, this function is used to divide all the rules in a multi-dimensional classifier into a set of buckets.

\section{(a) Definitions of Suml and Sum2}

Let $\alpha$ be a binary sequence and let $|\alpha|$ be used to express the length of $\alpha$. Assuming that $E L$ bits are extracted beginning from the leftmost bit in $\alpha$ for calculations, where $E L \leq|\alpha|$. The extracted binary string from $\alpha$ is expressed as $a_{1} a_{2} a_{3} \ldots a_{E L}$. We assign a weight to each extracted bit. The bit on the left (higher position) has higher weight than the bit on the right (lower position). Maximum weight $M W(M W \geq E L)$ is assigned to the left-most extracted bit, $a_{1}$. Minimum weight $M W-E L+1$ is assigned to the right-most extracted bit, $a_{E L}$. The difference of weights between any two adjacent bits is 1 .

Let $\operatorname{Sum} 1(\alpha, E L)$ be the sum of all the extracted bit values in $\alpha$. In short, $\operatorname{Sum} 1(\alpha, E L)=$ $\sum_{i=1}^{E L} a_{i}$. The value of $\operatorname{Sum} 1(\alpha, E L)$ represents the number of $1 \mathrm{~s}$ that occur in the extracted bits.

Let $\operatorname{Sum} 2(\alpha, E L, M W)$ be the sum of the products of each bit and its corresponding 
weight in the extracted sequence. In short, $\operatorname{Sum} 2(\alpha, E L, M W)=\sum_{i=1}^{E L} a_{i}(M W-i+1)$. The value of $\operatorname{Sum} 2(\alpha, E L, M W)$ reflects the distribution of all the 1s in the extracted bits.

Also, since the bit that occurs in $\alpha$ is either 1 or $0, \operatorname{Sum} 1(\alpha, E L)$ and $\operatorname{Sum} 2(\alpha, E L, M W)$ also can reflect the distribution of the $0 \mathrm{~s}$ in the extracted bits of $\alpha$.

For example, let $\alpha=01100010$ and $E L=M W=6$. Sum 1 and Sum 2 of $\alpha$ are:

$$
\begin{gathered}
\operatorname{Sum} 1(\alpha, E L)=0+1+1+0+0+0=2 \\
\operatorname{Sum} 2(\alpha, E L, M W)=6 \cdot 0+5 \cdot 1+4 \cdot 1+3 \cdot 0+2 \cdot 0+1 \cdot 0=9
\end{gathered}
$$

When $E L$ and $M W$ are fixed, sequence $\alpha$ is mapped into one definite pair of hash values (Sum 1, Sum2), which represents the distribution feature of the bits in the sequence. For example, when both $E L$ and $M W$ are 6, such two sequences, 01100010 and 10010001, share one pair of hash values, $(2,9)$. Therefore, the proposed hash function here is a more to one function, and there are collisions in some hash buckets.

When $E L$ and $M W$ are fixed, there are many sequences that are mapped into such a set of hash values ( $h, \operatorname{Sum} 2)$, where $h$ is a fixed value of $\operatorname{Sum} 1$ and all the possible values Sum 2 can take are a set of consecutive positive integers. Particularly,

$$
\begin{aligned}
& \operatorname{Minimal}\left(\left.\operatorname{Sum} 2(\alpha, E L, M W)\right|_{\text {Sum } 1=h}\right)=\frac{h(2 M W-2 E L+h+1)}{2} \\
& \operatorname{Maximal}\left(\left.\operatorname{Sum} 2(\alpha, E L, M W)\right|_{\text {Sun } 1=h}\right)=\frac{h(2 M W-h+1)}{2}
\end{aligned}
$$

Let NumSum 2 denote the number of all the possible values that Sum 2 can take, when Sum 1 is fixed. If Sum 1 is $h$, then NumSum $2=h(E L-h)+1$, which is a quadratic function. When $h=\left\lceil\frac{E L}{2}\right\rceil$ (or $\left\lfloor\frac{E L}{2}\right\rfloor$ ), NumSum 2 reaches its maximum value, $\frac{E L^{2}}{4}+1$. Let $\mathrm{NmSet}$ denote the number of all possible pairs of hash values when $E L$ is fixed. NmSet $=\sum_{i=1}^{E L}[i(E L-i)+1]=\frac{1}{6} E L\left(E L^{2}+5\right)+1$. For example, when $E L=8, N m S e t=93$, and when $E L=12, \mathrm{NmSet}=299$. 

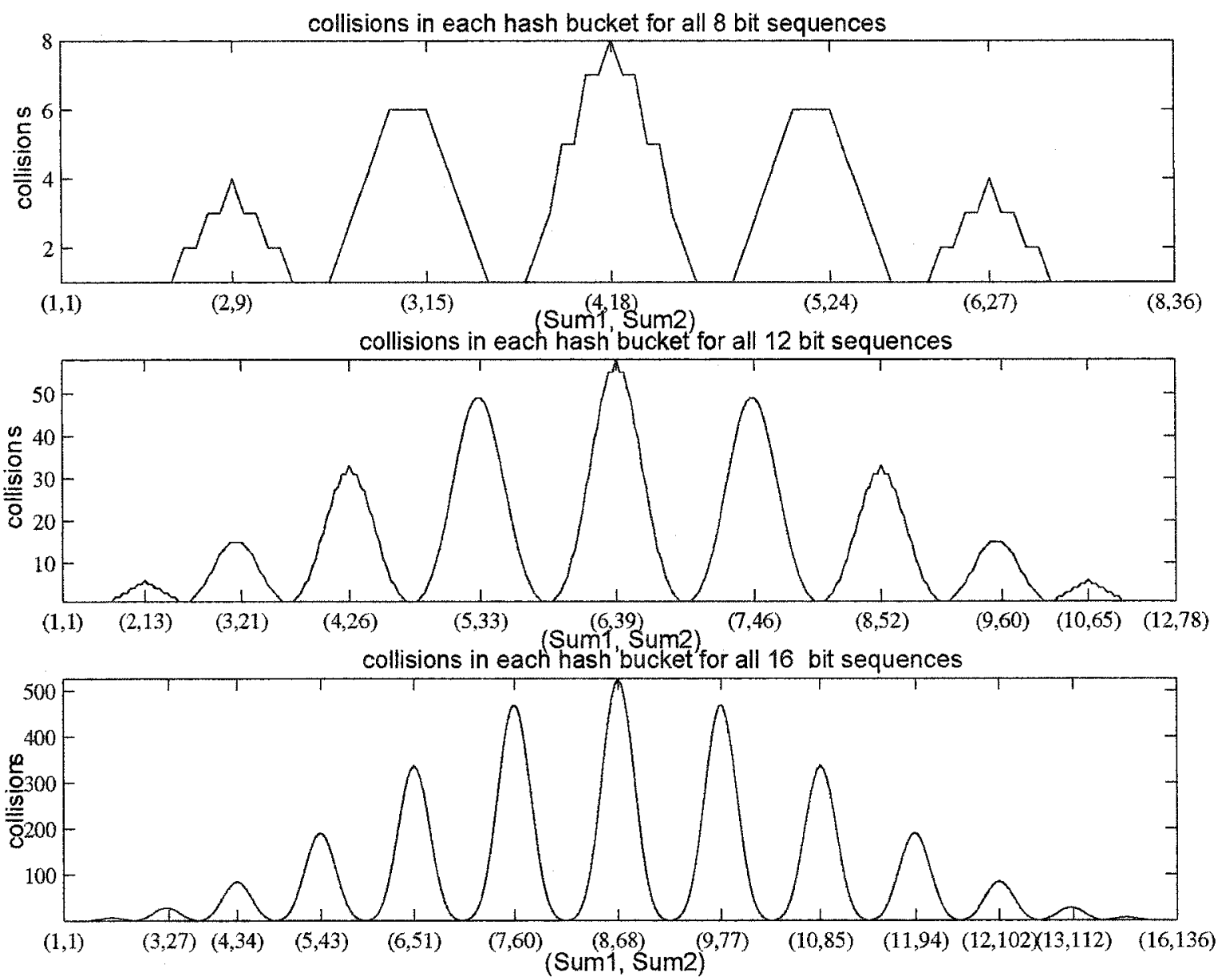

Figure 5.2: Hash results

Figure 5.2 depicts some distribution results of all $n$-bit long sequences after using the hash function (Sum1, Sum2). The number of the sequences in each hash bucket looks like a comb. With the growth of $E L$, its local maximum values also increase.

For any two pairs of hash values, $\left(\operatorname{Sum} 1_{1}, \operatorname{Sum} 2_{1}\right)$ and $\left(\operatorname{Sum} 1_{2}, \operatorname{Sum} 2_{2}\right),\left(\operatorname{Sum} 1_{1}\right.$, $\left.\operatorname{Sum} 2_{1}\right)$ is said to be smaller than $\left(\operatorname{Sum} 1_{2}, \operatorname{Sum} 2_{2}\right)$, if either of the two following conditions is satisfied: 
- $\operatorname{Sum} 1_{1} \leq \operatorname{Sum} 1_{2}$

- $\operatorname{Sum} 2_{1}<\operatorname{Sum} 2_{2}$, when $\operatorname{Sum} 1_{1}=\operatorname{Sum} 1_{2}$.

\section{(b) Sum1 and Sum2 of a prefix}

Assuming that $P$ is a prefix, and its prefix length is $P L$. Let $E L$ bits be extracted beginning from the leftmost of $P$ for calculations. Then $P$ is mapped into one or several pairs of hash values with respect to different values of $E L$.

- If $E L \leq P L$, then all the extracted bits have definite values, 0 or 1. Both $\operatorname{Sum} 1(P, E L)$ are $\operatorname{Sum} 2(P, E L, M W)$ are definite values. $P$ is mapped into one pair of hash values (Sum1,Sum2).

- If $E L>P L$, then the hash process is more complicated, for some extracted bits are not defined and they can be either 0 or 1 . The prefix $P$ is expanded into $2^{E L-P L}$ $E L$-bit long prefixes, named $P_{1}, P_{2}, \ldots, P_{2^{E L}-P L}$, in which $P_{1}=P \oplus 00 \ldots 00, P_{2}=$ $P \oplus 00 \ldots 01$ and $P_{2^{E L-P L}}=P \oplus 11 \ldots 11$. In order to determine which pairs of hash values $P$ can be mapped into, all $2^{E L-P L}$ expanding prefixes have to be considered. Each expanding prefix is mapped into a pair of hash values (Sum 1, Sum2) and some of them may share one pair. The minimum and the maximum values of Sum 1 and 
Sum 2 of $P$ that can reach are as follows:

$$
\begin{aligned}
\operatorname{Min}(\operatorname{Sum} 1(P, E L)) & =\operatorname{Sum} 1\left(P_{1}, E L\right) \\
\operatorname{Max}(\operatorname{Sum} 1(P, E L)) & =\operatorname{Sum} 1\left(P_{2} E L-P L, E L\right) \\
& =\operatorname{Sum} 1\left(P_{1}, E L\right)+E L-P L \\
\operatorname{Min}(\operatorname{Sum} 2(P, E L, M W)) & =\operatorname{Sum} 2\left(P_{1}, E L, M W\right) \\
\operatorname{Max}(\operatorname{Sum} 2(P, E L, M W)) & =\operatorname{Sum} 2\left(P_{2 E L-P L}, E L, M W\right) \\
& =\operatorname{Sum} 2\left(P_{1}, E L, M W\right)+\frac{1}{2}(E L-P L)(E L-P L+1)
\end{aligned}
$$

$P$ is duplicated into a set of hash values $(\operatorname{Sum} 1$, Sum 2$)$. Let ExpandSet denote the size of the duplication set. ExpandSet $=\frac{1}{6}(E L-P L)\left[(E L-P L)^{2}+5\right]+1$, which is controlled by the value of $E L-P L$.

For example, $P=011 *$ and its prefix length $P L$ is 3 . When both $E L$ and $M W$ are $5, P$ is expanded into 4 prefixes, 01100,01101, 01110, and 01111. These 4 expanded prefixes are mapped into 4 pairs of hash values $(2,7),(3,8),(3,9)$, and $(4,10)$. The $\operatorname{Min}(\operatorname{Sum} 1(P, E L))$ $=2, \operatorname{Max}(\operatorname{Sum} 1(P, E L))=4, \operatorname{Min}(\operatorname{Sum} 2(P, E L, M W))=7, \operatorname{Max}(\operatorname{Sum} 2(P, E L, M W))=10$ and ExpandSet $=4$.

(c) Sum 1 s and Sum2s Calculation in a 2-dimensional classifier, (Source IP and Destination IP)

Let $(S ; D)$ be the abbreviation for (Source IP address; Destination IP address) of a packet. $E L_{s}$ and $E l_{d}$ bits are extracted sequentially from $S$ and $D$ for calculation, respectively. Let $M W=M A X\left(E L_{s}, E L_{d}\right)$.

$$
\operatorname{Sum} 1(S ; D)=\operatorname{Sum} 1\left(S, E L_{s}\right)+\operatorname{Sum} 1\left(D, E L_{d}\right)
$$


$\operatorname{Sum} 2(S ; D)=\operatorname{Sum} 2\left(S, E L_{s}, M W\right)+\operatorname{Sum} 2\left(D, E L_{d}, M W\right)$

Let us investigate (Sum1, Sum 2) of an entry in a 2-dimensional classifier. Let $\left(P_{s}, P L_{s}\right.$; $\left.P_{d}, P L_{d}\right)$ be an entry in such a classifier, where $P_{s}$ and $P_{d}$ are the Source IP address prefix and Destination IP address prefix, respectively; $P L_{s}$ and $P L_{d}$ are the corresponding prefix lengths of $P_{s}$ and $P_{d}$. For simplicity, if we let $E L_{s}=E L_{d}=E L$, then $N m S e t=$ $\frac{1}{3} E L(E L-1)(E L-1)+\left(E L^{2}+E L+1\right)$.

When $E L$ is longer than either $P L_{s}$ or $P L_{d},\left(P_{s}, P L_{s} ; P_{d}, P L_{d}\right)$ will be mapped into multiple pairs of hash values, which causes the expansion. Figure 5.3 provides a mesh for the number of pairs of hash values to be mapped into based on values of $E L-P L_{s}$ and $E L-P L_{d}$. The number of the pairs reduces sharply when either $E L-P L_{s}$ or $E L-P L_{d}$ decreases. For example, if $E L-P L_{s}=8$, and $E L-P L_{d}=8$, then ExpandSet $=353$, While if $E L-P L_{s}$ is still 8, but $E L-P L_{d}$ is reduced to 4, then ExpandSet $=191$.

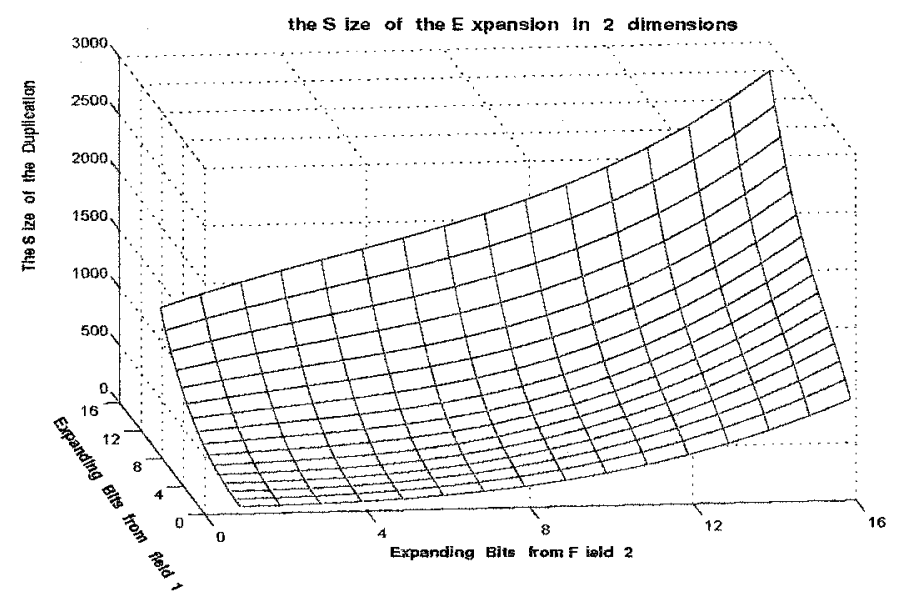

Figure 5.3: The Expansion for 2-Dimensional Entries

\section{(d) Sum1 and Sum2 Calculation in 5-dimensional classifier}


In general, a five-dimensional classifier is composed of the following fields: Source IP Address (32 bits), Destination IP Address (32 bits), Source Port (16 bits), Destination Port (16 bits), and Protocol ( 8 bits). Usually, the rules of the Source Port and Destination Port fields are range filters, but they can be expressed as a collection of prefix-based filters. For example, the interval $[0100,1001]$ can be decomposed into two prefixes $01^{*}$ and $100^{*}$. We will only describe the methods to calculate Sum 1 and $\operatorname{Sum} 2$ for 5-dimensional prefixbased filters for simplification. The Sum1 (entry) (or Sum2(entry)) is the sum of Sum 1 (or Sum2) over all fields.

Let $E x I P, E x P T$, and $E x P L$ be defined as the number of the bits extracted from $\mathbb{P}$ Address fields, Port fields and Protocol field for hash lookup respectively. Let $M W=$ $\operatorname{Max}(\operatorname{ExIP,ExPT,ExPL)}$. The weights assigned to the extracted bits of each field (from the lowest position to the highest position) are as follows:

IP Address: $M W-E x I P+1$ to $M W$;

Port: $M W-\operatorname{ExPT}+1$ to $M W$;

Protocol: $M W-E x P L+1$ to $M W$.

The larger weight always contributes more than the smaller weight does in Sum2 calculation. In order to allow the contribution of each field to Sums hash values to be in the same level, the same maximum weights are assigned to each field.

For example, if there is an entry from a 5-dimensional classifier (011000011001*, $\left.11000011101^{*}, 10001101^{*}, 00110110^{*}, 10100110\right)$ and if $\operatorname{ExIP}=10, \operatorname{ExPT}=8, \operatorname{ExPL}=$ 8 , then $M W$ is 10 . The weights of the IP Address are from 1 to 10 , the weights of the Protocol are from 3 to 10 , and the weights of the Ports are from 3 to 10. Then, $\operatorname{Sum} 1($ entry $)=4+5+4+4+4=21$ and Sum 2 (entry) $=22+28+24+24+27=125$. This entry is mapped into the pair of hash values $(21,125)$. It is obvious that the effects 
from all the fields on Sums hash values keep in balance.

In general, we can extract an arbitrary number of the bits from each field. As we know, in a rule, if the length of the extracted bits is longer than its prefix itself in any field, it will result in storage explosion, since that prefix has to be expanded to a set of longer prefixes and will be mapped into multiple pairs of hash values. The difference between the length of the extracted bits and the prefix controls the explosion size. When determining the lengths of the extracted bits for Sums calculations, we need to examine the bits in each field first, in order to make sure that the length of the extracted bits is not smaller than most lengths of prefixes of the rules to avoid storage explosion.

Let $L_{1}, L_{2}, L_{3}, L_{4}$ and $L_{5}$ be the lengths of the bits extracted beginning from the leftmost in those five dimensions. Let $M W$ equal to $\operatorname{Max}\left(L_{1}, L_{2}, L_{3}, L_{4}, L_{5}\right)$. The weight intervals occupied by all the fields are $\left[W M-L_{1}+1, W M\right],\left[W M-L_{2}+1, W M\right],[W M-$ $\left.L_{3}+1, W M\right],\left[W M-L_{4}+1, W M\right]$, and $\left[W M-L_{5}+1, W M\right]$ respectively.

$$
\begin{aligned}
& \operatorname{Min}(\operatorname{Sum} 1(\text { entry }))=0 \\
& \operatorname{Min}(\operatorname{Sum} 2(\text { entry }))=0 \\
& \operatorname{Max}(\operatorname{Sum} 1(\text { entry }))=L_{1}+L_{2}+L_{3}+L_{4}+L_{5} \\
& \operatorname{Max}(\operatorname{Sum} 2(\text { entry }))=\frac{1}{2}\left[(2 L M-1)\left(L_{1}+L_{2}+L_{3}+L_{4}+L_{5}\right)-\left(L_{1}^{2}+L_{2}^{2}+L_{3}^{2}+L_{4}^{2}+L_{5}^{2}\right)\right]
\end{aligned}
$$

\section{Data Structure}

A complete hash lookup process includes one Sum 1 table lookup and one Sum 2 table lookup. Each entry in the Sum 1 table records the information used to index into the Sum 2 table. A definite Sum 1 value can be indexed into the Sum 1 Table as a hash key. Each entry 


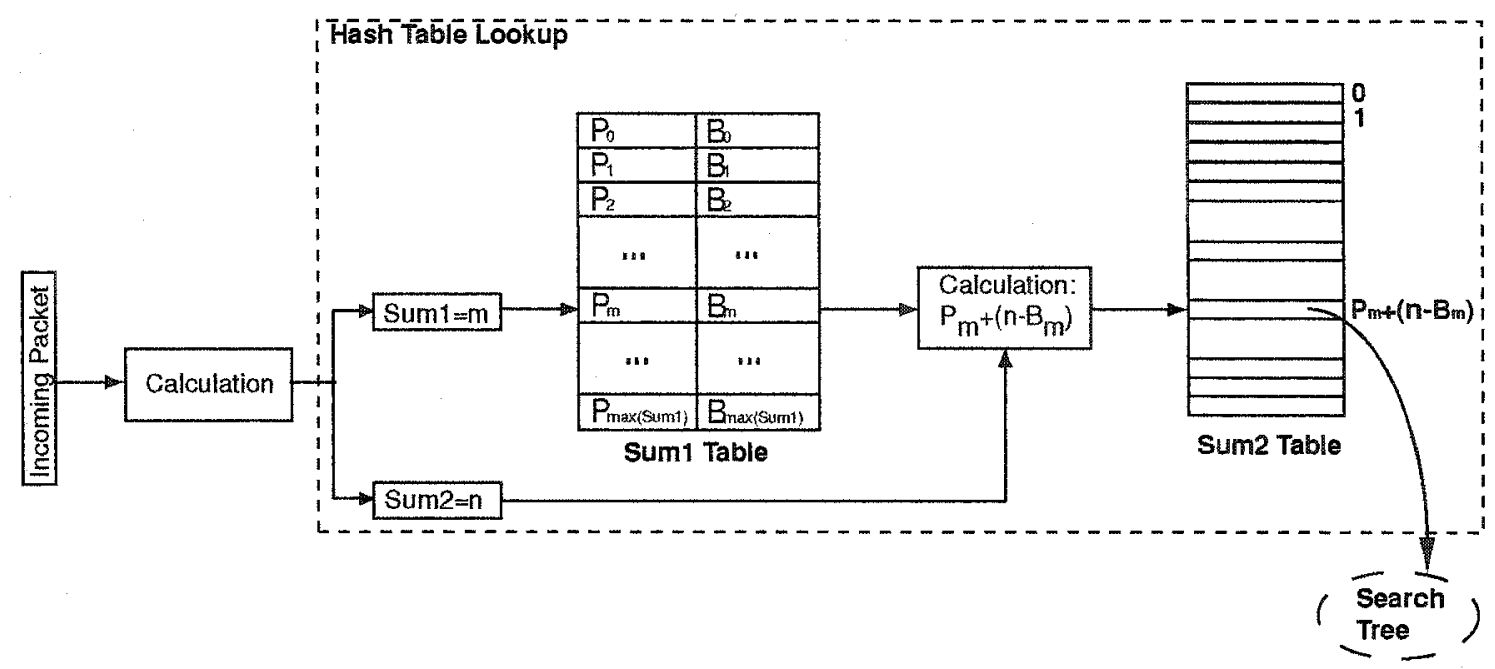

Figure 5.4: Hash Lookup Flow Chart

in the Sum 2 table denotes a pointer to a search trie. All the entries are sorted according to the hash values (Sum1, Sum2) in an increasing order.

As we know, when the $\operatorname{Sum} 1$ is fixed, say $\operatorname{Sum} 1=i$, there are a set of hash values that can be expressed as $(i, *)$, where $*$ means a set of consecutive values of Sum2. Therefore, the information of entry $i$ in the $\operatorname{Sum} 1$ table is composed of two components: $P_{i}$ and $B_{i}$. Let $P_{i}$ denote the beginning index of hash values $(i, *)$ in the Sum 2 table. Let $B_{i}$ be the value of $\operatorname{Min}(\operatorname{Sum} 2)$ when $\operatorname{Sum} 1=i$.

$$
\begin{aligned}
& P_{0}=0 \\
& B_{i}=\left.\operatorname{Min}(\operatorname{Sum} 2)\right|_{\operatorname{Sum} 1=i} ; \\
& P_{i}=P_{i-1}+\left(\left.\operatorname{Mox}(\operatorname{Sum} 2)\right|_{\operatorname{Sum} 1=i-1}-B_{i-1}+1\right), i>0
\end{aligned}
$$

In short, $P_{i}$ is the index of hash values $\left(\mathrm{i},\left.\operatorname{Min}(\operatorname{Sum} 2)\right|_{\operatorname{Sum} 1=i}\right)$ located in the $\operatorname{Sum} 2$ table. No hash value overlap in Sum 2 table.

If there is an incoming packet, after calculation, the pair of hash values $(m, n)$ are 
recorded. First, it is indexed into the $m^{\text {th }}$ entry in the $\operatorname{Sum} 1$ table and fetches $P_{m}$ and $B_{m}$.

Then, it is indexed into the $\left(P_{m}+\left(n-B_{m}\right)\right)^{t h}$ entry in the Sum 2 table to get the pointer to a specific search trie.

Figure 5.4 gives us a flow chart to describe how the hash lookup processing performs.

\subsubsection{Search Trie}

After the hash table lookup, the number of the rules, nRule, in each hash bucket narrows down rapidly. When nRule is larger than the predefined maximum size of a final small set (bucket size), LEAFSIZE, an optimal search trie is needed. The branching decision is made by inspecting some most significant bit positions at each step until the number of the rules may be contained in any node is no more than LEAFSIZE. Since some bits can not make much of the contribution in branching decision, they can result in big difference among the rules contained in child nodes, so that some leaves stay at very deep levels. In this subsection, we will describe how to determine the significant bit positions for branching decision at each node. An optimal tree must trade off these following demands: minimizing the mean steps to reach leaves, minimizing the nodes of the tree, and keeping the trie in a good shape.

In Figure 5.5, there is a set of sequences that are mapped into the pair of hash values (Sum $1=4, \operatorname{Sum} 2=24)$, where both $E L$ and $M W$ are 9. (A) illustrates a result when using the first 3 bits of the sequences in building a search tire. Six out of its eight children are empty, while each of the other two non-empty children contains three rules. (B) gives us a result when selecting the other 3 bits in building a search trie. Not only does the ratio of empty children nodes reduce, but also the maximum number of the rules in a child node 


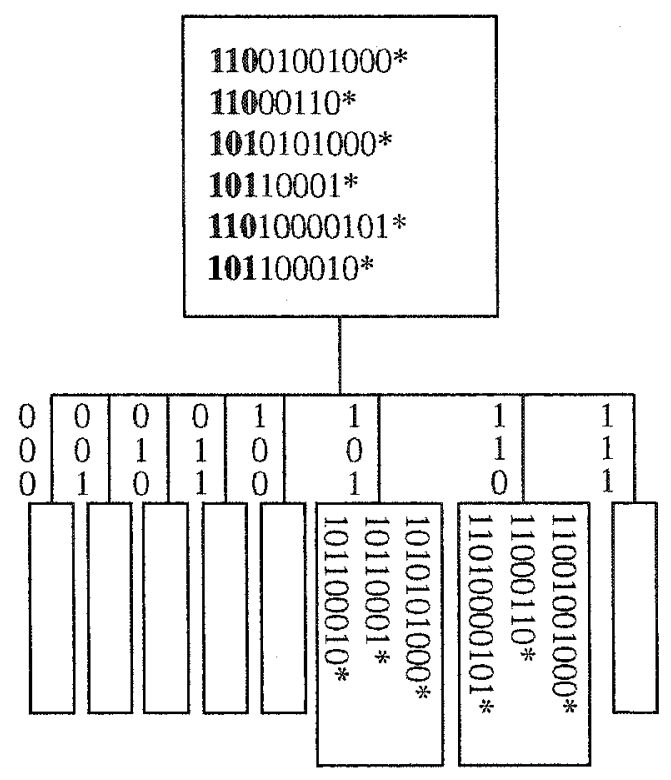

(A)

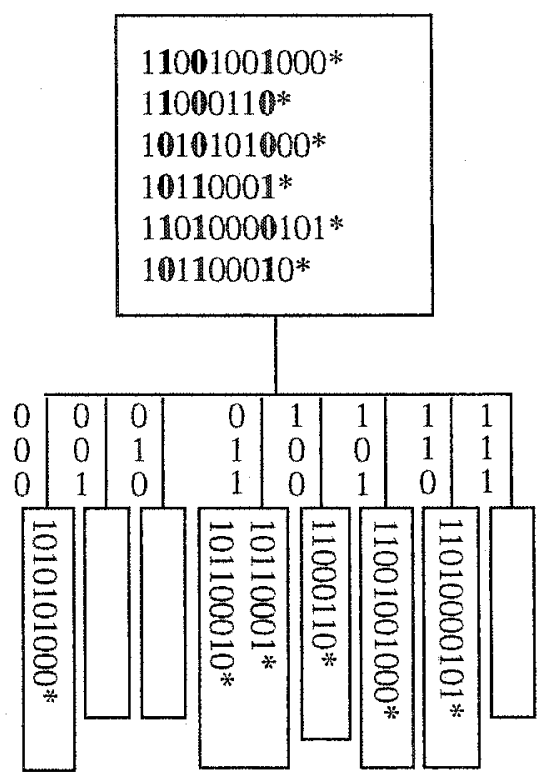

(B)

Figure 5.5: Comparison of Different Bit Selection Methods (1)

decreases. Therefore, the load for further procedures in each child node becomes more balanced.

Therefore, how to determine the most significant bits is a key step in building a search trie. A similar bit selection approach has been discussed in [59]. In our proposed algorithm, at each level, two main bit select steps are processed for a dynamic bit selection procedure.

\section{Elementary bit selection}

Elementary bit selection is similar to the one shown in [59]. All the rules in a classifier can be expressed by an array of long binary sequences. The length of the array, $N$, is the 
number of the rules. The width of array, $M$, depends on the dimensions. In IPv4, if it is a 2-dimensional classifier (Source IP address and Destination IP address), the width of the array is 64-bits long; while it is a 5-dimensional classifier, the width of the array is 104-bits long. We use a matrix $E(N$ rows by $M$ columns) to stand for the array, and each element $E(i, j)$ is 0,1 , or *.

For each column $j(1 \leq j \leq M), 3$ quantities, $N u m_{0, j}, N u m_{1, j}$ and $N u m_{*, j}$ are defined as follows:

$N u m_{x, j}=\sum_{i=1}^{N}(E(i, j)==x)$, where $x$ could be 0,1, or *. In short, $N u m_{x, j}$ is the total number of rows whose $j^{\text {th }}$ column is $x$.

Furthermore, we define $D_{j}$ as the difference between the total numbers of 0 s and $1 \mathrm{~s}$ the $j^{\text {th }}$ column contains. In short, $D_{j}=\mid N u m_{0, j}-$ Num $_{1, j} \mid$,

We also denote the smallest and the largest values of $D_{j}$ among all the columns of $E$ by $D_{\min }$ and $D_{\max }$, respectively. $N u m_{*, \min }$ and $N u m_{*, \max }$ are defined to express the smallest and the largest values of $N u m_{*, j}$ among all the columns of $E$.

Preference $[j]=\frac{D_{j}-D_{\min }}{D_{\max }-D_{\min }}+\frac{N u m_{*, j}-N u m_{*, \min }}{N u m_{*, \max }-N u m_{*, \min }} ;$

The Preference $[j]$ is the combination function of $D_{j}$ and $N u m_{*, j}$. The smaller value of $D_{j}$ means that it may lead to a more equal amount of rules into child nodes of the next level. But the branches based on bit $j$ will not eliminate more rules from consideration for the amount of traffic proportional to $N u m_{*, j}$. Hence, in order to maximize the progress benefit, the value of $\mathrm{Num}_{*, j}$ should be minimized.

Assume that the number of the rules in a classifier is $N$. After the hash table lookup procedure, the number of rules in hash bucket $k$ will be decreased to $N_{k}\left(N_{k} \ll N\right)$. Only when $N_{k}$ is larger than $L E A F S I Z E$, it is required to build a multi-way search trie. The ideal branching is the one that not only keeps the depth of the search trie lower, but it also 
allows the number of the rules in each leaf to be close to $L E A F S I Z E$.

First, we need to calculate how many significant bits should be inspected at each node in theory. Let a parameter MAXBITS denote the maximum number of the significant bits that can be inspected at a node. We define NBits $=\min \left(\frac{\log _{2} \frac{N_{k}}{L E A F S I Z E}}{\operatorname{la}^{\prime}}, M A X B I T S\right)$, where NBits is calculated to determine the number of significant bits, and notation $\bar{a}$ denotes the nearest integer to $a$. Therefore, it can meet the demand that the number of the rules in each child node at the next level is close to LEAFSIZE. Otherwise, the number of the rules in the child node is either too small, which is not efficient in storage consumption and can lead to memory explosion, or too big, which is not efficient in access times and can cause to longer branches in the trie.

Then, we will describe the elementary bit selection process. A pre-defined factor, PreBound $(<1)$, is pre-defined. Any bit position $j$, whose Preference $[j]$ is no more than PreBound, will be selected for discussion. Let $N D$ be the set of selected bit positions. We will discuss the elements in the set $N D$ as follows.

- If $|N B i t s|=1$, then any bit position with the smallest Preference can be chosen. The bit selection process of this node terminates.

- If $|N D| \leq N$ Bits \& $|N B i t s|>1$, then all the bit positions in $N D$ are set as the most significant bits. The bit selection process of this node terminates.

- If $|N D|>$ NBits \& $|N B i t s|>1$, then let the NBits ${ }^{\text {th }}$ smallest Preference value be expressed by $C V$. The elements, $j$, in the $N D$ are divided into three collections.

$$
j \in\left\{\begin{array}{l}
L C V, \text { when Preference[j] }<\mathrm{CV} \\
E C V, \text { when Preference[j] }=\mathrm{CV} \\
G C V, \text { when Preference[j] }>\mathrm{CV}
\end{array}\right.
$$


All the columns (bit positions) belonging to $L C V$ are determined as the selected bits. The columns (bit positions) belonging to $E C V$ are the candidates for precise bit selection. The columns (bit positions) belonging to $G C V$ will be ignored.

Since after hash function is performed, the rules in one hash bucket have "regularities" in their bits distribution. The differences among the Preference values of the bits chosen in NBits may be either big that can not be ignored or 0 . In that case, it needs precise bit selection.

\section{Precise Bit Selection}

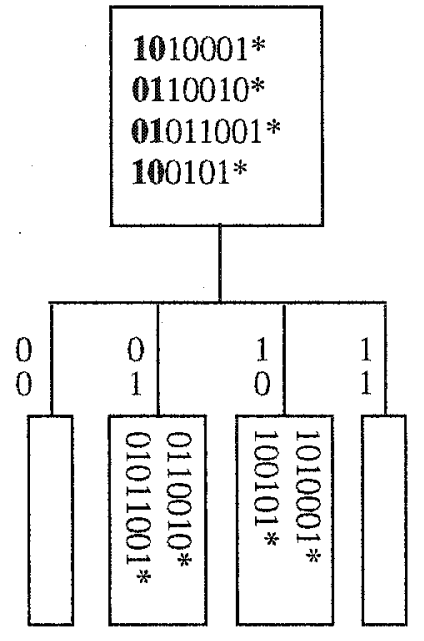

(A)

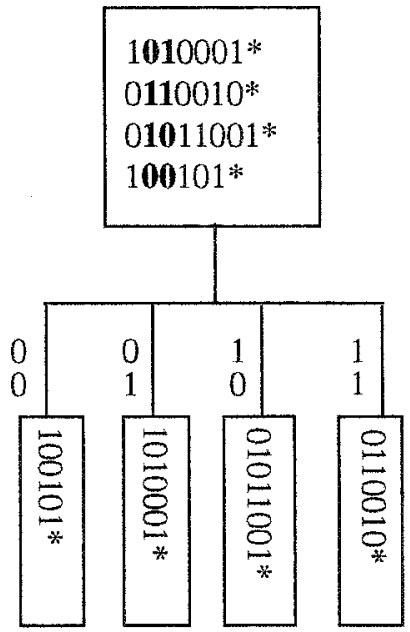

(B)

Figure 5.6: Comparison of Different Bit Selection Methods (2)

Figure 5.6 illustrates a comparison between two different bit selection methods. There is a set of four sequences falling into one pair of hash values $(\operatorname{Sum} 1=3, \operatorname{Sum} 2=13)$, where 
both $E L$ and $M W$ are 7. We define $L E A F S I Z E=1$ and $M A X B I T S=2$. After calculations, $D_{\text {min }}=0, D_{\max }=1$, Num $_{* \text { min }}=0$, and $N u m_{*, \max }=1$. The Preferences of the $1^{\text {st }}, 2^{\text {nd }}$, $3^{\text {rd }}, 4^{\text {th }}$, and $6^{\text {th }}$ bit positions beginning from the leftmost are all 0 . Actually, 2 bits are enough to distinguish them, for $\min \left(\overline{\log _{2} \frac{N_{k}}{L E A F S I Z E}}, M A X B I T S\right)=\min \left(\overline{\log _{2} \frac{4}{1}}, 2\right)=2$. How to determine the 2 bits out those 5 significant bits? (A) gives us the result when the $1^{\text {st }}$ bit and the $2^{\text {nd }}$ bit of the prefixes are chosen to build a search trie. One half of the child nodes are empty, while each of the other two nodes contains two prefixes. So one more division step is needed to make sure that no more than LEAFSIZE rules are contained in a leaf. Both the memory and the search time are wasted. It also causes to the same result when the $3^{\text {rd }}$ bit and the $4^{\text {th }}$ bit are chosen. (B) provides a better result when the $2^{\text {nd }}$ bit and the $3^{\text {rd }}$ bit are chosen as the most significant bits. Only one division step is processed to meet the requirement that the number of the rules in each leaf is not larger than LEAFSIZE. From this example, it is clear to see that a better bit selection strategy can effectively improve the search performances.

The target of this step is to choose (NBits $-|L C V|$ ) significant bits from the set of $E C V$, if $E C V$ is not empty and (NBits $-|L C V|)>0$. Both small mean and small standard deviation of the amount of the rules in its child nodes are desirable.

Let $S_{i}$ be a set of possible bit positions chosen from $E C V$, where $i \leq\left(\begin{array}{c}|E C V| \\ N B i t s-|L C V|\end{array}\right)$ and $\left|S_{i}\right|=N B i t s-|L C V|$. Let $L C V \oplus S_{i}$ denote each bit position combination of $L C V$ and $S_{i}$. Let $S t d_{i}$ stand for the standard deviation of the number of the rules distributed to its child nodes based on $L C V \oplus S_{i}$. The bits combination $L C V \oplus S_{k}$ is chosen to be the best match, when the corresponding $S t d_{k}$ is the minimal one. This precise bit selection process is needed at each node in the search trie, as long as all the following conditions are satisfied (1) node contains more than LEAFSIZE rules, (2) ECV is not empty and (3) NBits is 
larger than $|L C V|$.

\section{Data Structure}

The data structure of the nodes in the search trie is as follow:

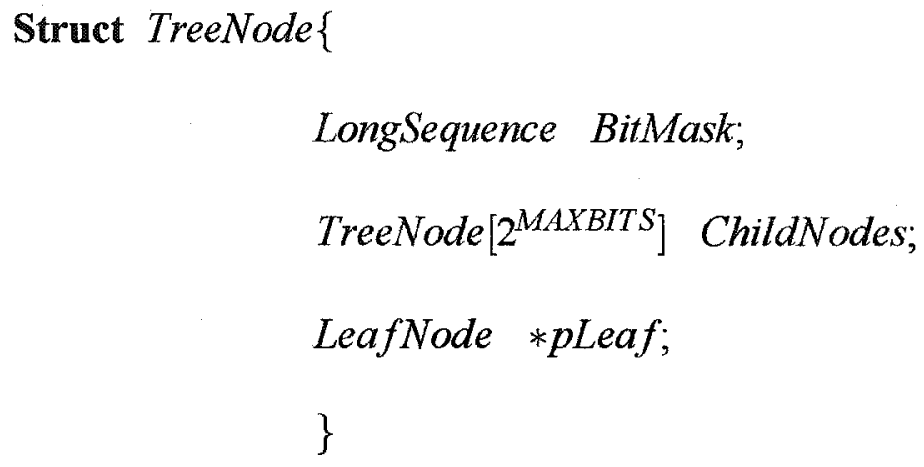

BitMask is an $M$-bit long sequence, where $M$ depends on the dimensions. For instance, $M$ equals to 64 for 2-dimensional address classifiers in IPv4. The $i^{\text {th }}$ bit is set to 1 if and only if it is chosen as one of the most significant bits, otherwise it is set to 0. If the node is a leaf, then its BitMask is set to NULL. ChildNodes is defined as an array of pointers to its children nodes. The size of the array is set $2^{\text {MAXBITS }}$, due to its maximum number of the child nodes. Any pointer in the array can be set to NULL, if the corresponding branch is empty. All those pointers are set to $N U L L$ when this node is a leaf. pLeaf is used as a pointer to the final small set, when it is a leaf, otherwise it is set to $N U L L$.

\subsubsection{Small Set}

The basic unit in the proposed algorithm is the leaf node of the search trie. Every leaf 
node contains a small set of rules and has the following properties. Firstly, it contains a small pre-defined maximum number (LEAFSIZE) of rules; typical sizes of it are 8, 16,32 . Secondly, the rules are similar in that there is a set of bit positions such that all the rules in it are consistent. Since the number of the rules in such a set is small, many techniques can be used to search in it efficiently. Linear search and binary search are two examples. In linear search, the indices of the rules in the leaf are stored according to the rule priorities in decreasing order. We then fetch the indices pointed by the leaf and use the indices one by one to get the rule for comparing with the incoming packet until a matching rule is found. Since the set of rules is very small, the search can easily be sped up by the pipelined or parallel solution.

\subsection{Updates}

In this dynamic packet classification algorithm, we need to update the algorithm structure to accommodate the changes in the packet classification table. The basic update algorithm is intuitive, similar procedure to lookup. When adding a rule, we use the (Sum1, Sum2) hash function to locate the corresponding buckets. Then in each bucket, the bits at the specific positions direct the rule to a leaf (or leaves). If the addition results in overflow in any leaf, it is required to make further division, so that the number of the rules in each of its child nodes is no more than LEAFSIZE. When deleting a rule, it is the opposite process. This update may make the structure nonoptimal, for some particular branches of the search trie are very long, or the search trie will not keep in good shape, which will result in inefficient data structure. We need to recreate a new search trie from the root of 
the hash bucket, whose search trie is not in a good shape. How often we need to recreate the data structure can be decided by applications.

Extendable hash table grows or shrinks as the database grows or shrinks. It allows inserting and deleting rules easy implemented. It is a heuristic idea to combine extendable hash table with our proposed SUMs hash function, to improve the performance of updates in this algorithm.

\subsection{An Example}

Table 5.1: A Small Two-Dimensional Classifier

\begin{tabular}{|l|l|l||l|l|l|}
\hline No. & Field 1 & Field 2 & No. & Field 1 & Field 2 \\
\hline 1 & $00^{*}$ & $00^{*}$ & 7 & $10^{*}$ & $01^{*}$ \\
\hline 2 & $00^{*}$ & $111^{*}$ & 8 & $10^{*}$ & $111^{*}$ \\
\hline 3 & $000^{*}$ & $100^{*}$ & 9 & $101^{*}$ & $001^{*}$ \\
\hline 4 & $001^{*}$ & $100^{*}$ & 10 & $101^{*}$ & $100^{*}$ \\
\hline 5 & $010^{*}$ & $001^{*}$ & 11 & $11^{*}$ & $110^{*}$ \\
\hline 6 & $011^{*}$ & $01^{*}$ & 12 & $110^{*}$ & $1011^{*}$ \\
\hline
\end{tabular}

Table 5.1 is a simple 2-dimensional classifier with 12 entries. After examining the classifier, we define $E L_{1}=E L_{2}=M W=2$. Then, the $S u m 1$ Table has 5 entries and the Sum2 Table has 9 entries, as shown in Figure 5.7. The 12 rules are divided into 8 hash buckets. We also define $L E A F S I Z E=1$ and MAXBITS $=2$. Then an optimal search trie is built in each hash bucket if it contains more than one rule. Figure 5.7 also illustrates how 


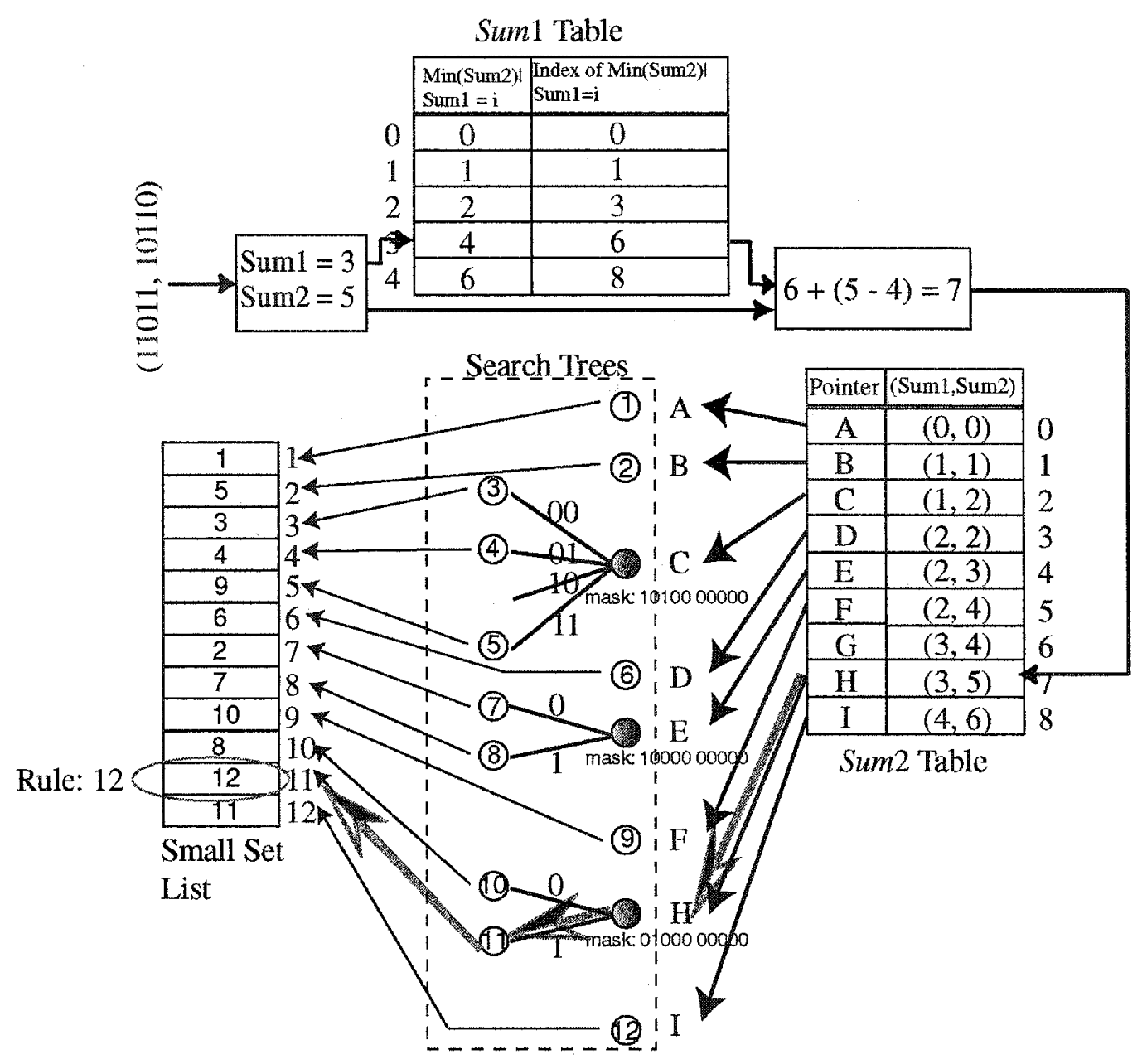

Figure 5.7: An Example for 2-Dimensional Packet Classification 
the proposed packet classification algorithm proceeds when there is an incoming packet (11011, 10110).

\subsection{Performance Analysis}

The performance of the proposed algorithm will be discussed in this section. The algorithm is applied in two types of classifiers: 2-dimensional classifiers, which are composed of two fields: IP Source Address Prefix and IP Destination Address Prefix; and 5dimensional classifiers, which are composed of five fields: Source IP address, Destination IP address, Protocol, Source Port, and Destination Port. These classifiers are generated based on the base prefixes from the Mae-east and Aads.

\subsubsection{Performance for 2-Dimensional Packet Classification}

Hash table lookup and search trie lookup are two major optimal lookup steps in this proposed algorithm. In the following part of this subsection, we will analyze the performance of (Sum1, Sum2) hash function first (We call it and its hash table as SUMs hash function and SUMs hash table for short.). Then the total system performance combined with search tries will be discussed next.

(a) The feature of the SUMs Hash Table

This proposed new heuristic packet classification algorithm has a similar basic structure to the algorithm proposed in [59], which also organizes the search space into 3 layers, 
JUMP table, search trie, and filter bucket. The JUMP table is built with respect to the JUP hash function, in which the first $a$ bits and the first $b$ bits are concatenated respectively from each field to form $a 2^{a+b}$ long JUMP table. In order to verify the performance of the SUMs hash table, we compare it with the JUMP table.

Let" $a$ by $b$ " express extracting the first $a$ bits and the first $b$ bits from the prefixes in field 1 and field 2 , respectively. The bits increment pattern is from " 3 by 4 ", " 4 by 4 ", " 4 by 5 ", $\ldots$ to " 16 by 16 ". Since the JUMP table explodes with the increase of $a$ and $b$, $a$ and $b$ are confined to be no more than 10 , especially $b$ is limited to be no more than 9 when the number of rules is $512 \mathrm{~K}$, in building the JUMP table.

The SUMs hash table is composed of the Sum 1 table and the Sum 2 table. The Sum 1 table is very small. The incremental growth of the Sum 1 table is only two entries, when the number of the extracted bits is increased by 1 in each field. It is much more stable and shorter, compared with the incremental growth of the Sum 2 table. What we discuss in this section will be concentrated on the Sum2 table only.

Figure 5.8 describes the table lengths for the SUMs hash function and the JUMP hash function in a 2-dimensional classifier. We can see that the SUMs hash table is smaller and more stable, compared to the JUMP hash table, proposed in [59]. When the first 16 bits are extracted from each field to build a hash table, the SUMs hash table contains no more than $4 \mathrm{~K}$ entries, while the JUMP hash table contains $1 \mathrm{G}$ entries. The JUMP hash table reaches $4 \mathrm{~K}$ when both $a$ and $b$ are only 6 . Considering that the JUMP hash function is a one-to-one function and the SUMs hash function is a many-to-one function, the size of the JUMP hash table is much more sensitive to the number of the extracted bits. Assume that $E x$ bits are extracted from each field to build the hash table. Once the number of the extracted bits from each field is increased to $E x+1$, then the incremental 


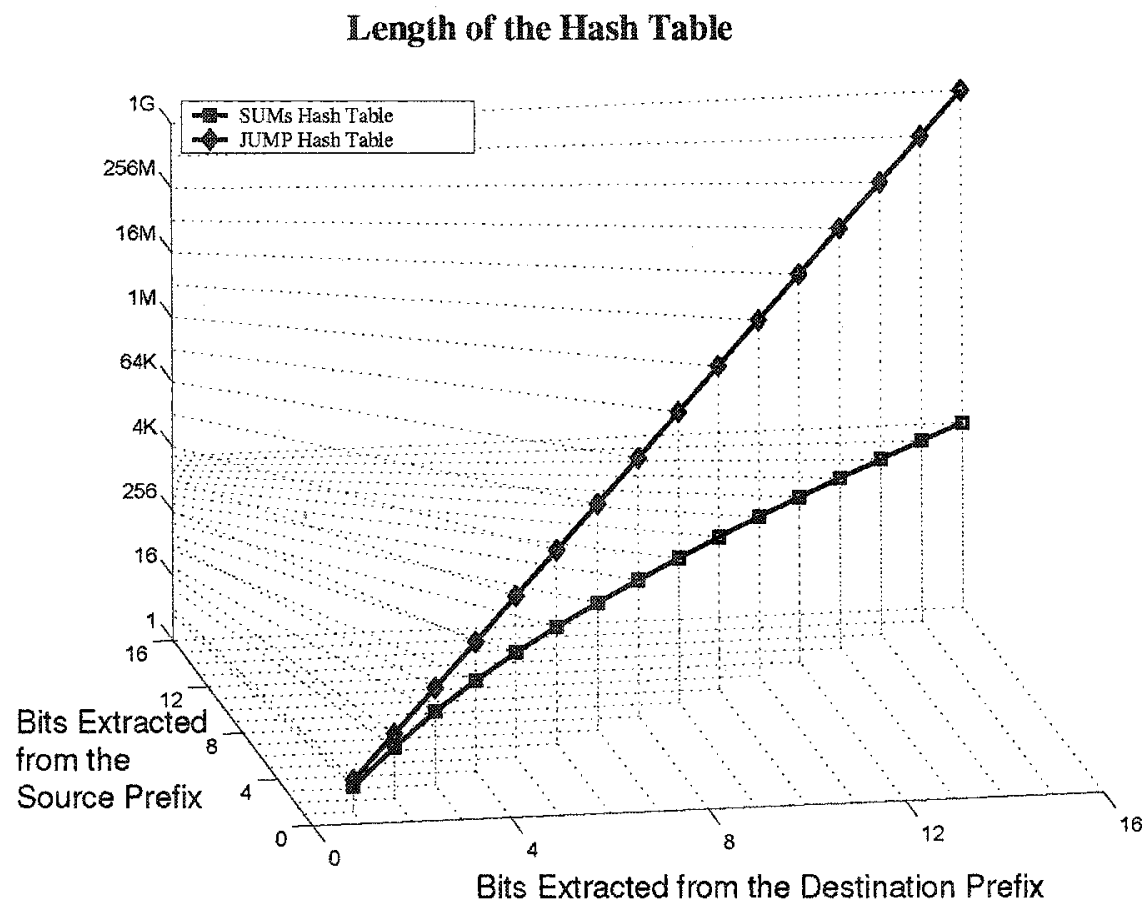

Figure 5.8: Length of the Hash Table

growth of the SUMs(Sum2) hash table is $2\left(E x^{2}+E x+1\right)$, which is a quadratic increasing function, while the incremental growth of the JUMP hash table is $3\left(2^{E x}\right)$, which is an exponentially increasing function. In short, when the number of the extracted bits from any field increases, the increasing rate of the SUMs hash table is much slower than the JUMP hash table. The lengths of IPv4 address prefixes are usually longer than 8 bits, and most of them are between 16 bits to 24 bits. So, SUMs hash function makes it possible to extract more bits to generate a hash table, to allow bit distribution feature more sufficiently represented, so that it can reduce the collision in each bucket.

The merits derived from Sums hash function can be summarized into the following four aspects. 


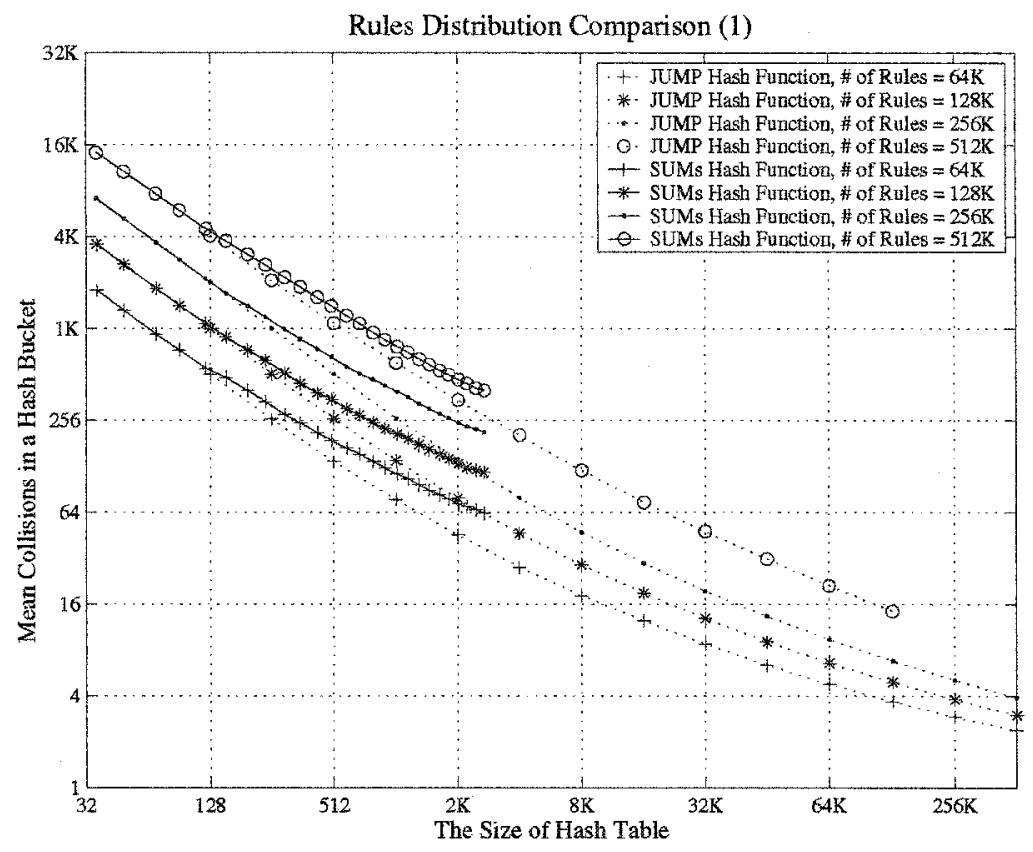

Figure 5.9: Mean Collisions in a Hash Bucket

Firstly, the SUMs hash table only consumes a very small amount of memory. Even when 16 bits are extracted in each field, it costs no more than $16 \mathrm{~K}$ bytes, which is small enough to be stored in cache (or on-chip SRAM memory). It is helpful to speed up the hash lookups.

Secondly, if the number of the extracted bits is larger than a Destination/Source prefix length in any field, then the corresponding prefix has to be duplicated into a set of hash buckets. But the storage explosion of the SUMs hash table is much smaller than that of the JUMP hash table. Considering a rule, assuming that its prefixes in both fields expand 8 bits, then the rule will be duplicated into 353 buckets of the SUMs hash table (shown in Figure 5.3), while the duplication in the JUMP hash table is $2^{16}$.

Thirdly, it can overcome the unbalanced prefix distribution problem. The colliding 


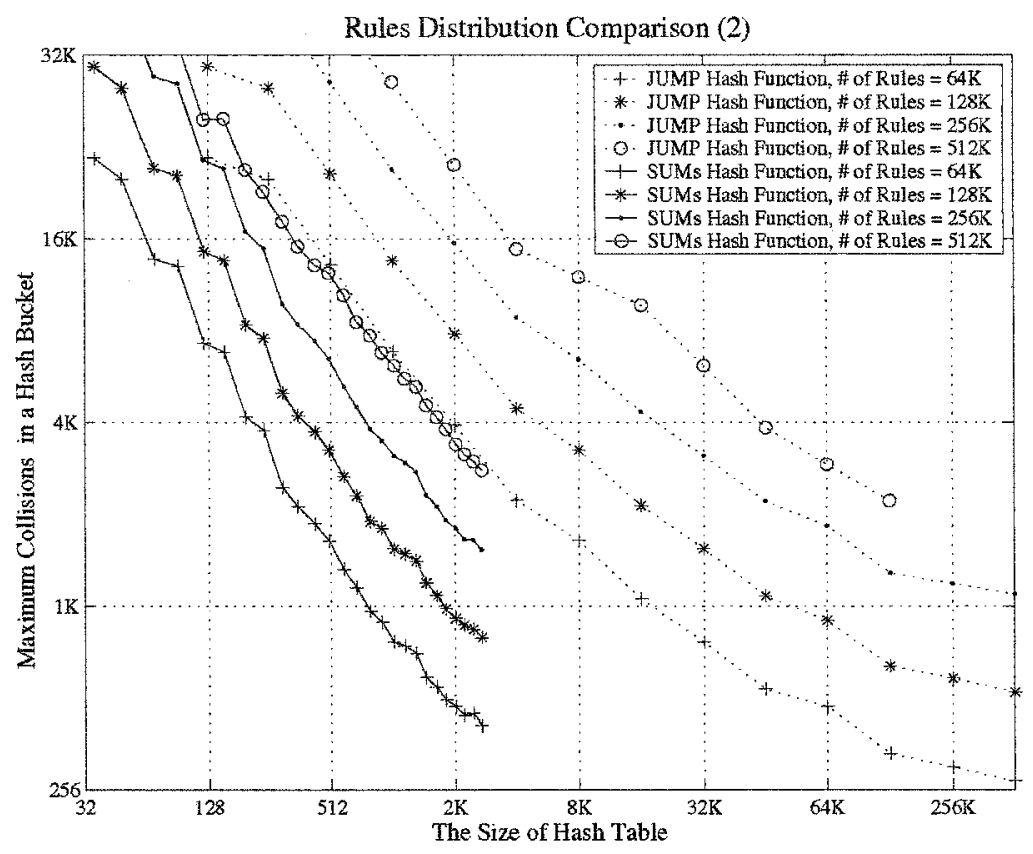

Figure 5.10: Maximum Collisions in a Hash Bucket

rules in one SUMs hash bucket may not share a common prefix in any dimension, due to the feature of the SUMs hash function. If the prefixes are not uniformly distributed, it will result in a big variance in the collisions in every hash bucket as compared to the JUMP hash table. Figure 5.9 and Figure 5.10 compare the distributions of the collisions in each hash bucket between the SUMs table and the JUMP table. The horizontal axis is used to denote the length of the hash table. Each marked point in the graph is the experiment results in terms of the the extracted bits " $a$ by $b$ ". The goal of the comparison is to evaluate the performance of these hash functions when the lengths of (SUMs/JUMP) hash tables are almost the same. It shows that the mean collisions in the SUMs hash bucket is slightly higher than that of the JUMP hash table in Figure 5.9. The reason is that when the SUMs table and JUMP table are almost the same, the bits used to build the SUMs table are more 
than the bits used to build the JUMP table, which causes the higher probability of bit expansion. However, the maximum collisions in a SUMs hash bucket is much smaller than that in a JUMP hash bucket in Figure 5.10.

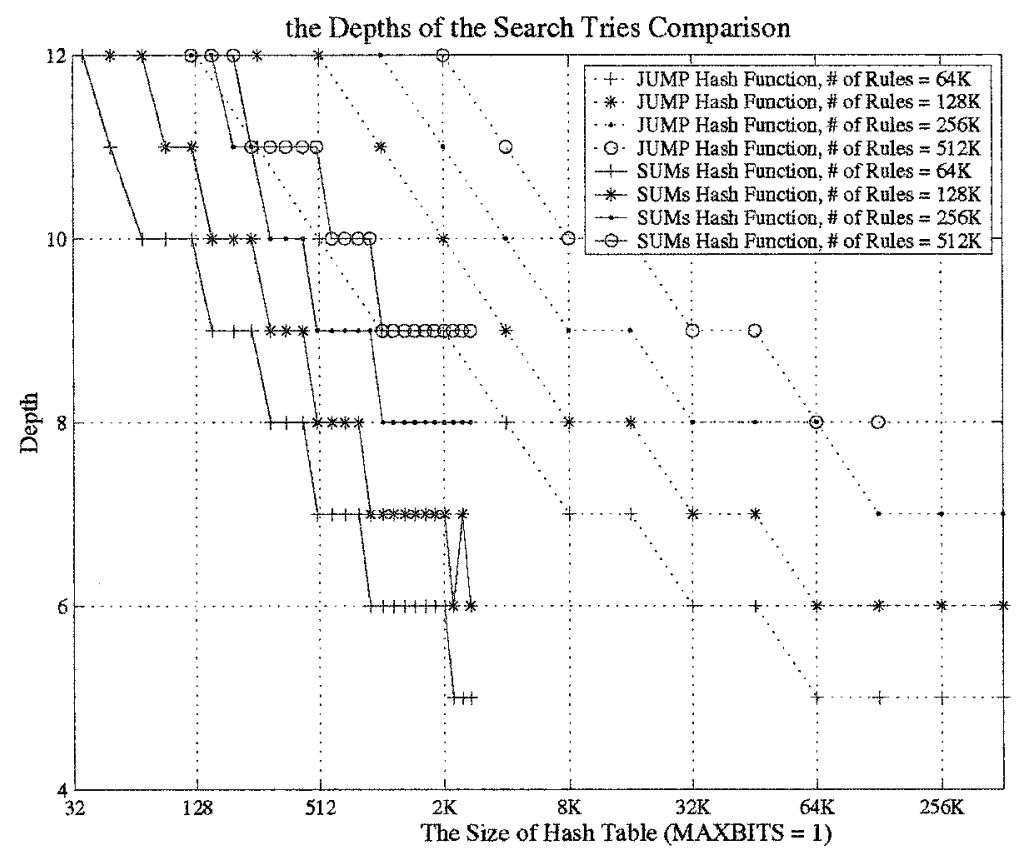

Figure 5.11: Depth of the Search Trie

Fourthly, the depth of the search trie of each hash bucket can benefit from the SUMs hash table. SUMs hash function can overcome the prefix distribution problem and it is capable of lowering down the maximum collisions among all the hash collisions. The depth of the search trie is lower than the search trie built with respect to the JUMP hash function. Figure 5.11 illustrates the depths of the search tries comparison between the SUMs hash function and the JUMP hash function. Let MAXBITS and LEAFSIZE be 1 and 16 , respectively. When the sizes of the JUMP hash table and SUMs hash table are almost the same, the depth of the search trie in terms of the SUMs hash table is around 3 
levels shorter than the one in terms of the JUMP hash table.

\section{(b) The performance of the total system}

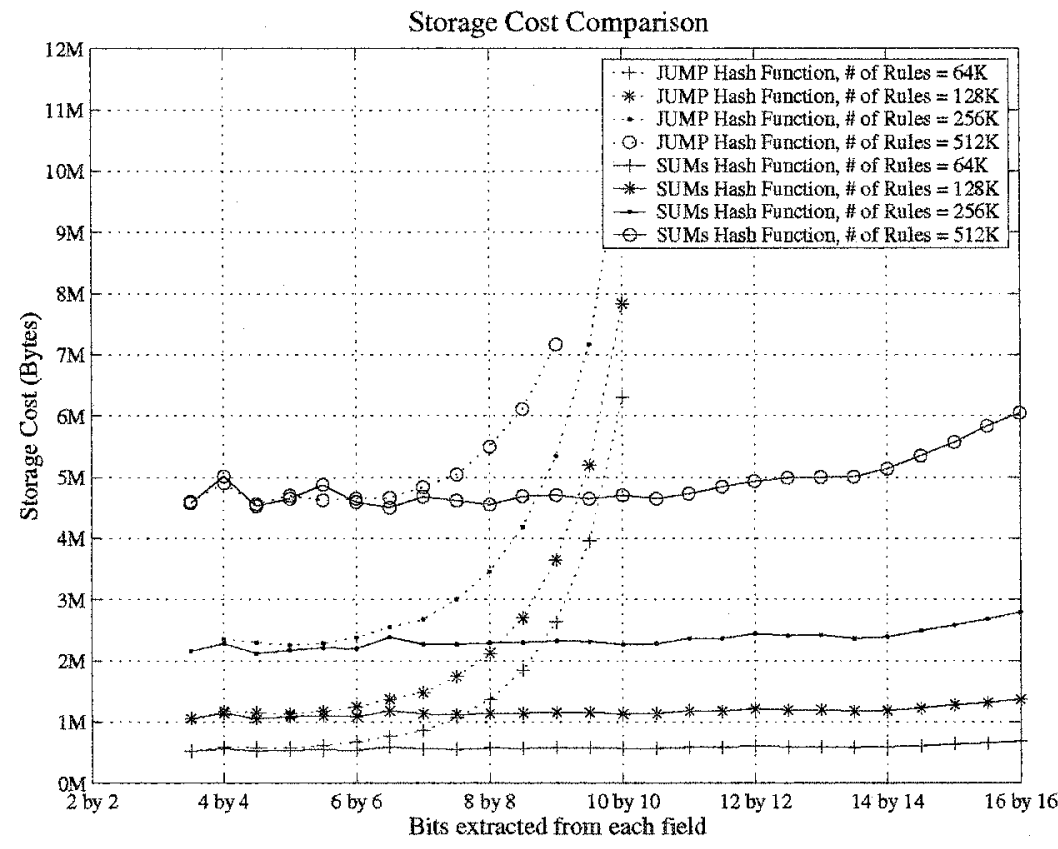

Figure 5.12: Storage Cost Comparison Between Using Different Hash Functions

This proposed heuristic packet classification algorithm based on bit characteristics is a memory saver. The storage cost comes from three resources: hash table, search trie and small sets. When using SUMs hash method, the length of the hash table can not exceed $4 \mathrm{~K}$. Therefore search tries and small sets are the main contributors to storage cost. Figure 5.12 compares the storage consumptions between using distinct hash functions. The horizontal axis denotes the bits extracting strategy from both fields. The storage cost in terms of the SUMs hash table is much steadier in the variation of the extracted bits. When " 12 by 12 " is used for the SUMs hash table, the storage cost of the total system is 
comparable with the cost of using " 6 by 6 " for the JUMP hash table.

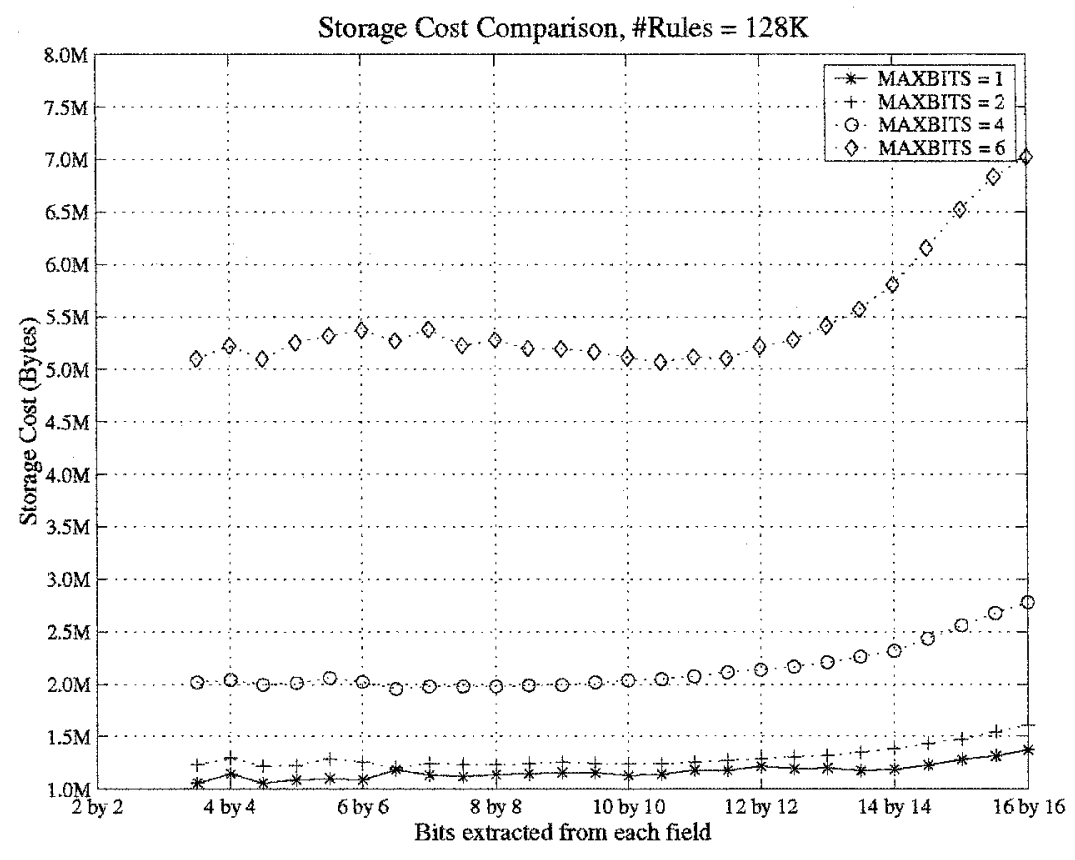

Figure 5.13: Comparison of the Storage Cost When MAXBITS Varies

Figure 5.13 evaluates the storage consumption when MAXBITS varies. For each individual curve, the slope of it climbs up rapidly once the extracted bits are more than " 12 by 12". While investigating all the curves, we can find that the storage cost increases with the growth of MAXBITS. The mainly increase of the storage cost is resulted from the nodes consumed in the search tries. Although MAXBITS is very big, the number of branches is also controlled by the parameter PreBound. Many of the nodes can not reach $2^{\text {MAXBITS }}$ children nodes actually, however each node consumes more storage, which is wasteful.

When the length of the hash table is fixed, let us examine the mean of the search steps till reaching a final small set in the search trie for random traffics. Figure 5.14 verifies that the search steps vary with the parameter MAXBITS by using the SUMs hash 


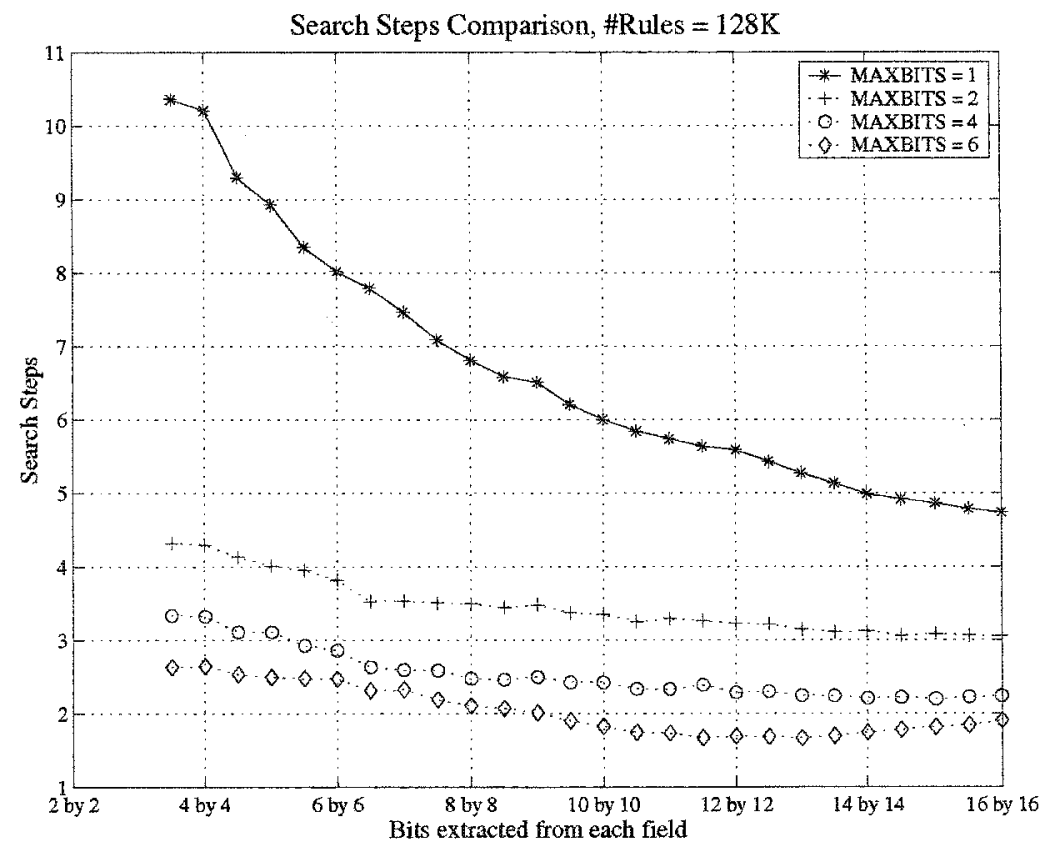

Figure 5.14: Comparison of the Search Steps When MAXBITS Varies

function. When MAXBITS is larger than 1, the search steps are reduced sharply, however, the reducing rate decreases with the growth of MAXBITS.

We can draw the conclusion that the multi-way search trie is beneficial to the search performance. But we can also see that when MAXBITS is larger than 4 , it will not reduce the search steps significantly, while the storage cost bursts. It is not worth choosing a very big MAXBITS. That let MAXBITS be 4 is ideal respect to our experimental case.

Wildcard rules are the main contributors toward storage explosion. If a dimension contains a large percentage of wildcard rules, it is better to separate them out, to build additional two 1-dimensional tables to avoid the storage explosion. Therefore, one 2dimensional searching system is converted to one 2-dimensional searching system with two additional smaller 1-dimensional search systems. These three systems must all be 
searched to find a match with the highest priority.

Suppose that $p$ percent of the $M$ rules contain wildcards, in which $p_{1}$ percent of the rules are in the Source field and $p-p_{1}$ percent of the rules are in the Destination field have wildcards. Let $L$ be the number of the bits extracted respectively from each field. Those rules with wildcards are duplicated into $\frac{L}{6}\left(L^{2}+5\right) p M$ rules during SUMs hash division. If the duplication of the rules is expected to be less than $\alpha M$, where $\alpha$ is a small number, to maintain the performance of the algorithm, it is necessary to separate out the wildcard rules when $p>\frac{6 \alpha}{L\left(L^{2}+5\right)}$. In contrast, considering the JUMP hash function, the wildcard rules have to be separated out when $p>\frac{\alpha}{2^{L}}$. We can prove that $\frac{6 \alpha}{L\left(L^{2}+5\right)}>\frac{\alpha}{2^{L}}$, for any possible $L$. Hence, the JUMP hash function is more sensitive to the percentage of wildcards. For example, let $p=25 \%$, and $\alpha=8$. In order to avoid separating the system, $L$ cannot be more than 6 in the SUMs hash process, while $L$ must be no more than 5 in the JUMP hash method.

In summary, the algorithm of packet classification using bits characteristics performs well for large 2-dimensional classifiers, no matter whether the prefix distribution is balanced or not, as long as the percentage of wildcard rules is not too big.

\subsubsection{Performance for 5-Dimensional Packet Classification}

The 5-dimensional classifiers we use here are synthetic ones based on the prefixes from Mae-east and Aads. The filters in the address fields are randomly selected from the 2-dimensional classifiers above. Let the filters in the port fields (or the protocol fields) and their prefix lengths be normal random variables. They are randomly selected from the ranges of $[0,65535]$ and $[0,16]$ (or $[0,255]$ and $[0,8]$ ) respectively. 
First, we examine the size of the SUMs hash table in terms of the extracted bits from each field. In Table 5.2, it is clear to see that the increasing rate of the SUMs hash table is much steadier than that of the JUMP hash table when the number of extracted bits increases. Therefore, it is possible to extract more bits for the hash function from each field, as long as the number of the extracted bits is no more than most prefix lengths in any field.

Table 5.2: Hash Table Size

\begin{tabular}{|l|l|l|l|l|l|l|}
\hline \multicolumn{5}{|c|}{ Extracted bits from each field } & Length of & Length of \\
Source & Dest. & Protocol & Source & Dest. & the SUMs & the JUMP \\
Address & Address & & Port & Port & Hash Table & Hash Table \\
\hline 4 & 4 & 4 & 0 & 0 & 103 & $4 \mathrm{~K}$ \\
4 & 4 & 0 & 4 & 4 & 177 & $16 \mathrm{~K}$ \\
8 & 8 & 8 & 4 & 4 & 1333 & $1 \mathrm{G}$ \\
8 & 8 & 8 & 8 & 8 & 2141 & $256 \mathrm{G}$ \\
10 & 10 & 8 & 4 & 4 & 2045 & $\ldots$ \\
16 & 16 & 8 & 4 & 4 & 6037 & $\ldots$ \\
\hline
\end{tabular}

Next, we will examine how the rules are distributed into the hash buckets. In our experiments, the filters in the Port fields and Protocol field are artificial normally distributed random variables. The mean and the standard deviation of the filters in the Port fields are 32767 and 3000 . The mean and the standard deviation of the prefixes of the filters in the Port fields are 10 and 2. The mean and the standard deviation of the filters in the Protocol 
field are 127 and 30 . All the protocols are 8 bit long.

Table 5.3: Subentries Distribution Using SUMs Hash Function (\# of Rules = 256K)

\begin{tabular}{|c|c|c|c|c|c|c|}
\hline \multicolumn{5}{|c|}{ Extracted bits from each field } & \multirow{2}{*}{$\begin{array}{l}\text { Mean Collisions } \\
\text { in a Hash Bucket }\end{array}$} & \multirow{2}{*}{$\begin{array}{l}\text { Max Collisions } \\
\text { in a Hash Bucket }\end{array}$} \\
\hline $\begin{array}{l}\text { Source } \\
\text { Address }\end{array}$ & $\begin{array}{l}\text { Dest. } \\
\text { Address }\end{array}$ & Protocol & $\begin{array}{l}\text { Source } \\
\text { Port }\end{array}$ & $\begin{array}{l}\text { Dest. } \\
\text { Port }\end{array}$ & & \\
\hline 8 & 8 & 0 & 4 & 4 & 583 & 4446 \\
\hline 8 & 8 & 8 & 4 & 4 & 428 & 3301 \\
\hline 8 & 8 & 8 & 8 & 8 & 440 & 3927 \\
\hline 10 & 10 & 8 & 4 & 4 & 296 & 2287 \\
\hline 16 & 16 & 8 & 4 & 4 & 144 & 1094 \\
\hline
\end{tabular}

From Table 5.3, it is obvious that the performance of the hash function depends on the extracted bits. The collision in a hash bucket declines with the increasing of the extracted bits in any field. However, in any field, when the number of the extracted bits is not longer than most prefixes, it will result in storage explosion in the hash process. For example, when the number of the extracted bits in the Port fields is increased to 8 from 4 , and the number of the bits extracted from other fields are fixed, either the mean collision or the maximum collision in the buckets increases, which is the opposite of what we expected. The reason is that the number of the rules whose port prefix lengths are smaller than 4 is no more than $11 \%$, according to Chebyshev's inequality, while there are many port prefix lengths smaller than 8 . Therefore, examining the sequences in each field first to find a set of suitable number of extracted bits is a key in our approach. 
Table 5.4: Algorithm Performance $(\#$ of Rules $=128 \mathrm{~K}, M A X B I T S=4, L E A F S I Z E=16)$

\begin{tabular}{|c|c|c|c|c|c|c|c|}
\hline \multicolumn{5}{|c|}{ Extracted bits from each field } & \multirow[t]{2}{*}{ Storage Cost } & \multirow{2}{*}{$\begin{array}{c}\text { Mean } \\
\text { Bits Examined }\end{array}$} & \multirow{2}{*}{$\begin{array}{c}\text { Mean } \\
\text { Search Steps }\end{array}$} \\
\hline $\begin{array}{l}\text { Sour. } \\
\text { Addr. }\end{array}$ & $\begin{array}{l}\text { Des. } \\
\text { Addr. }\end{array}$ & Protl & $\begin{array}{l}\text { Sour. } \\
\text { Pt }\end{array}$ & $\begin{array}{c}\text { Des. } \\
\text { Pt }\end{array}$ & & & \\
\hline 8 & 8 & $\overline{0}$ & $\overline{4}$ & 4 & 2.10MByte & 4.82 & 2.39 \\
\hline 8 & 8 & 8 & 4 & 4 & 2.22MByte & 4.78 & 2.41 \\
\hline 8 & 8 & 8 & 8 & 8 & 3.59MByte & 4.83 & 2.41 \\
\hline 10 & 10 & 8 & 4 & 4 & 2.31MByte & 4.33 & 2.20 \\
\hline 10 & 10 & 8 & 8 & 8 & 3.93MByte & 4.63 & 2.35 \\
\hline 16 & 16 & 8 & 4 & 4 & 3.28MByte & 3.87 & 1.87 \\
\hline
\end{tabular}

Table 5.4 describes the experiment performance results of $128 \mathrm{~K}$ classifiers. It shows that after the hash process, only a few most significant bits are needed to be inspected to reach the final small set. The SUMs hash function makes a big contribution in the new approach. It is suitable for higher dimensional classifiers. The best bits extracting strategy is " $10,10,8,4,4$ ", considering the combination of the storage cost and search performance.

The preprocessing procedures of our approach when the classifier contains many wildcards are as follows:

1. Examine all the rules in the classifier and determine the number of the extracted bits in each field to build the SUMs hash table. It should meet the requirement that the number of the extracted bits should be smaller than most prefixes in the dimension which we are observing (except for the wildcards).

2. Observe the wildcards in $K$-dimensions and decide whether the percentage of the wildcards is so big that it is necessary to separate them out to build additional reduced-sized systems. If it is, selecting a dimension, which contains the most 
wildcards, and separating those wildcard rules out is the optimal behavior. Then, an additional $K-1$ dimensional reduced classifiers are built. In our experiment, if the percentage of the wildcards is $50 \%$ of $256 \mathrm{~K}$ rules, 1 full size classifier and 3 additional reduced-size classifiers (one has 4-dimensions and two have 3-dimensions) are enough to decompose the rules so that the upper bound of wildcards in each system can meet the demand.

3. Build the Hash table and the dynamic search trie for each system.

The algorithm is suitable for parallel implementation. The systems may work in parallel or pipeline to find the best matching rule.

\subsection{Summary}

In this chapter, we presented a new packet classification algorithm, which is designed for big classifiers and is suitable for edge classifiers. Our approach combines three lookup procedures: a heuristic hash lookup, a novel multi-way dynamic search trie lookup and a lookup procedure for searching through fixed size rule sets. This architecture construction is motivated by practical observation, aimed at finding the best matching rule by traversing an optimal search path. It is an efficient way to take advantage of the bit characteristics of all the rules.

Through experiments with a large number of classifiers, we demonstrated that our approach constrained the maximum collision in each hash bucket effectively. It possesses advanced capabilities in fast search and small storage requirement. It is also scalable to multidimensional classifier and to IPv6. The disadvantage of our algorithm is that it is 
a little bit sensitive to wildcards, but it is still much better than the JUMP hash function. When the wildcards reach an upper bound, it is necessary to separate out those rules with wildcards. Then a full size system and a few reduced size systems are needed to process together to find the best matching rule. 


\section{Chapter 6}

\section{Applications of Efficient TCAMs in}

\section{Table Lookups}

In this chapter, we will describe some techniques to improve the performance of using TCAMs in table lookups. The main idea is to address the solutions to decreasing the power consumption, increasing the table size that a TCAM can handle, and avoiding entries expansion in range matching.

\subsection{Introduction}

A Ternary Content Addressable Memory (TCAM) is a fully associative memory that allows a "don't care" state for each memory cell, in addition to 0 and 1 . A memory cell in a "don't care" state matches both 0 s and $1 \mathrm{~s}$ in the corresponding input bit. TCAM's archi- 
tecture is simple and easy to understand, so that it allows several companies to produce compatible products. TCAM is ideal to all the matching processes except range matching. TCAM's performance is deterministic in small table lookups. It takes the same number of cycles to complete every single processing. There are no worse case scenarios that would take longer time [41]. This feature makes them particularly attractive for packet classification and IP address lookup applications, when we require the best matches. Moreover, TCAM-based tables are typically much easier to manage and update.

However, TCAMs have some limitations, such as high cost to density ratio, high power consumption, and poor range matching ability. These limitations impede their utilization in IP address lookups and packet classifications. Fortunately, both the density and the cost of TCAMs have dramatically improved in the past few years. There are commercially available TCAMs with density up to $18 \mathrm{M}$ bits per chip, which can store up to 512K 32 bit prefixes. However, current high-density TCAM devices consume 12 to 15 Watts per chip when the entire memory is enabled for search [69]. There are many references $[12,25,26,27,33,43,57,69]$ that proposed methods of using TCAMs in lookup problems.

In this chapter, we focus on the problem of making TCAM-based forwarding engines more efficient by exploiting commonly available TCAM features. First, some TCAM vendors now provide mechanisms for searching only a part of the TCAM device in order to reduce power consumption during a lookup operation. We can take advantage of this feature to provide power efficient TCAM-based architectures for IP lookup and packet classification. Then, we will propose an entry compacting method that is easy to implement and update in TCAMs. At last, we also try to solve range matching problem in packet classification by transforming it into exact matching under arbitrary mask problem. 


\subsection{Reducing TCAMs Power Consumption - Segment En-}

\section{ablement}

In this section, a two-stage efficient TCAM based search algorithm with its experiment results will be proposed. We focus on solving 1-dimensional table lookup problems - IP address lookups. But, it is also scalable to multi-dimensional table lookups.

The main contribution of this section are in two aspects. Firstly, a novel method to partition the forwarding table is provided, which allows small difference among the numbers of the entries in all segments. It is an efficient way to restrict the maximum power consumption of the TCAM. Also, the bits needed for matching in each cell of the TCAM are reduced, which provides another possible way to increase the size of a forwarding table a TCAM can handle based on available TCAM dense capability. This partition method also performs well for IP address lookup at the edge. Secondly, we evaluate our partitioning method using real forwarding table traces obtained from various core routers in the Internet. We show that in realistic settings, the power savings are guaranteed in the worst case.

A heuristic method is proposed in [69]. The core idea of it is to split the entire routing table stored in TCAM into multiple buckets and the lookup is a two-stage operation where a fixed set of bits from the first 16 bits in the input is to hash to one of the buckets, and then the lookup in the selected bucket is processed in the second stage. But the pre-processing time of bit selection is not efficient, since the selection method is exhaustive. Another two-stage TCAM lookup method is presented in [43] that uses the first 8 bits to enable one of the buckets in the second stage, which is not efficient enough. 
We will present a technique to choose the most significant bit positions among all 32 bits in IPv4. The main idea of it is to partition the entire forwarding table into several segments according to the bit values at the selected positions, so that the numbers of the entries in all segments are very close, and its standard deviation is very small. The target is to minimize the maximum entries in one segment. The architecture of the proposed algorithm is shown in Figure 6.1. At the first stage, $n$ most significant bits are selected as a key to be indexed into a segment address table. It is used to enable one of the segments of the TCAM. At the second stage, the left $32-n$ bits are sent to the TCAM for the comparison in a segment.

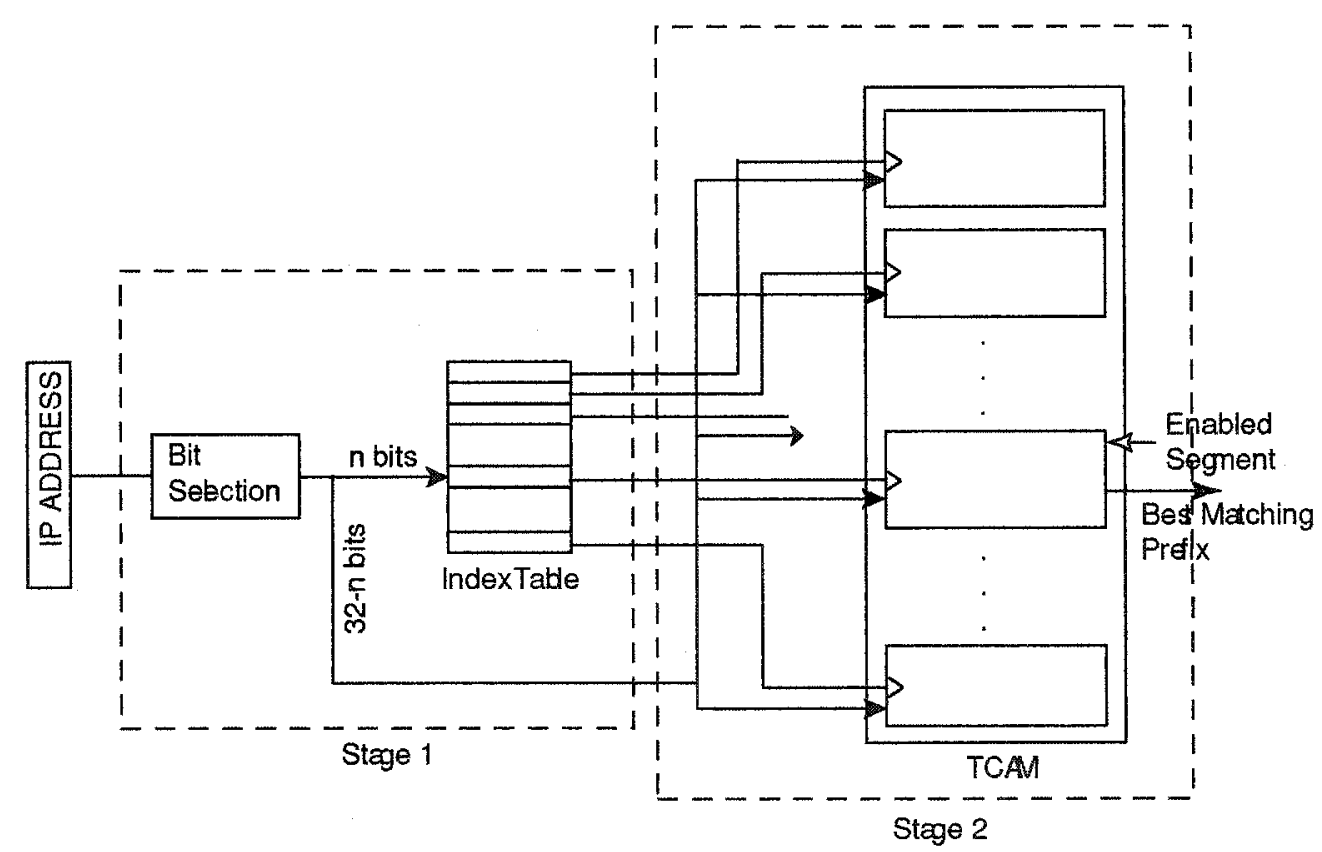

Figure 6.1: Proposed Architecture for TCAM-Based IP Address Lookup

The method to select the significant bits is the key process. In our proposed algorithm, there are two selection processes, elementary bit selection process and precise bit 
selection process.

\subsubsection{Elementary Bit Selection}

This elementary bit selection method is similar to the one in the previous chapter. Suppose that all the entries are partitioned into $2^{n}$ segments. Those $n$ bit positions with the smallest Preferences are chosen as the most significant bits. In theory, the power consumption decreases with the growth of $n$. But, more entry expansions may arise with the growth of $n$. It is necessary to choose a better value of $n$. In general, $n$ is defined to be no greater than 8 .

Table 6.1: Results of Using the Elementary Bit Selection

\begin{tabular}{|l|l|l|l|l|l|}
\hline Table & $\begin{array}{l}\text { No. of orig- } \\
\text { inal entries }\end{array}$ & $\begin{array}{l}\text { No. of Entries } \\
\text { Stored in TCAM }\end{array}$ & $\begin{array}{l}\text { Max Enabled } \\
\text { Entries }\end{array}$ & $\begin{array}{l}\text { Standard } \\
\text { Deviation }\end{array}$ & $\begin{array}{l}\text { Saved } \\
\text { Power }\end{array}$ \\
\hline Aads & 63980 & 79124 & 720 & 75.58 & $98.87 \%$ \\
Mae-East & 47206 & 66449 & 413 & 42.96 & $99.13 \%$ \\
Mae-West & 56032 & 63570 & 462 & 51.43 & $99.18 \%$ \\
Paix & 22116 & 24213 & 290 & 34.27 & $98.69 \%$ \\
\hline
\end{tabular}

The result of selecting 8 significant bits by using the elementary bit selection process is shown in Table 6.1. When selecting 8 bits, the forwarding table is partitioned into 256 segments. The expected entries in each segment is $0.4 \%$ of the total and it will save $99.6 \%$ of the power consumption. From Table 6.1, we can see that even the minimal saved power is more than $98.6 \%$. 
In Table 6.2, it gives us the corresponding results when selecting the first 8 bits as the key for the partition. The standard deviation of the entries in each segment is much higher than the value in Table 6.1. Even the maximal saved power consumption is no more than $91.2 \%$. It is not efficient enough for the power consumption problem in TCAM. From this aspect, the merit of the bit selection cannot be overlooked.

Table 6.2: Result of Selecting the First 8 bits to Split the Forwarding Table

\begin{tabular}{|l|l|l|l|l|l|}
\hline Table & $\begin{array}{l}\text { No. of } \\
\text { entries }\end{array}$ & $\begin{array}{l}\text { Sum of Entries } \\
\text { in TCAM }\end{array}$ & $\begin{array}{l}\text { Max Enabled } \\
\text { Entries }\end{array}$ & $\begin{array}{l}\text { Standard } \\
\text { Deviation }\end{array}$ & $\begin{array}{l}\text { Saved } \\
\text { Power }\end{array}$ \\
\hline Aads & 63980 & 63980 & 7454 & 1389.13 & $88.35 \%$ \\
Mae-East & 47206 & 47206 & 6599 & 1002.56 & $86.02 \%$ \\
Mae-West & 56032 & 56032 & 5557 & 1256.43 & $90.08 \%$ \\
Paix & 22116 & 22116 & 1952 & 480.79 & $91.17 \%$ \\
\hline
\end{tabular}

\subsubsection{Precise Bit Selection}

After the elementary bit selection, some bit positions with the smaller Preferences are chosen. But sometimes the values of the Preferences are too close to determine which bit is more suitable. It is also hard to decide whether those selected bits still can perform well in partition when they cooperate as a key. In the precise bit selection process, we will decide the best cooperating bits.

Assuming that the final partition key is $S$ bits long. After elementary bit selection, $T(S<T)$ bits are chosen as the candidates. In general, $(T-S)$ is less than 4 . The target 
of this step is to choose the best $S$-bit combination as a partition key from $T$ candidates, in that both the variance and the entry duplication in all partition segments are small. In total, we will examine $\left(\begin{array}{l}T \\ S\end{array}\right)$ partition key candidates to find the best set. For each candidate, the numbers of the entries falling into each one of the $2^{S}$ segments are examined. The small standard deviation of the entries in each segment and the small value of the entries duplication are desirable. The proposed algorithm here is simpler than the one in [69].

All the possible $\left(\begin{array}{l}T \\ S\end{array}\right)$ partition key candidates are sorted. Let $\mathcal{C}$, an array with $\left(\begin{array}{l}T \\ S\end{array}\right)$ rows and $S$ columns, denote the possible selected bit positions of all the candidates. Each row of $C$ is a set of partition key candidate. Each element in it denotes a bit position. $\mathcal{C}_{i, j}$ records the value of the $j^{\text {th }}$ selected bit position in the $i^{\text {th }}$ key candidate.

Let Entry $y_{i}^{j}$ denote the $j^{t h}$ bit of the $i^{t h}$ entry in a forwarding table, which can be 0,1 , or * Let $a^{l}$ represent the $l^{\text {th }}$ bit of value $a$. Let $\mathcal{E}$ be an array with $\left(\begin{array}{l}T \\ S\end{array}\right)$ rows and $2^{S}$ columns. Let $\mathcal{E}$ represent the numbers of entries belonging to all possible partition segments. Each element of $\mathcal{E}$ records the number of entries falling into one certain partition segment with respect to a fixed partition key (bit positions).

$$
\begin{aligned}
& \mathcal{E}(l, j)=\sum_{i=1}^{N}\left(\operatorname{Entry} y_{i}^{C_{l, k}}==\left(j^{k} \text { or }{ }^{*}\right), \forall k, k \leq S\right) ; \\
& S T D_{i}=\sqrt{\sum_{j=1}^{2^{S}}(\mathcal{E}(i, j)-\overline{\mathcal{E}(i,:)})^{2}} ; \\
& \text { SumEntry }{ }_{i}=\sum_{j=1}^{2^{S}} \mathcal{E}(i, j) ;
\end{aligned}
$$

$S T D_{\min }$ and $S T D_{\max }$ represent the minimum and the maximum value of all $S T D(i)$, respectively. Similar, SumEntry $y_{\min }$ and SumEntry $y_{\max }$ represent the minimum and the maximum value of all SumEntry(i), respectively.

$$
\text { Preference } 2[i]=\frac{S T D_{i}-S T D_{\min }}{S T D_{\max }-S T D_{\min }}+\frac{\text { SumEntry }_{i}-\text { SumEntry }_{\min }}{\text { SumEntry }_{\max }-\text { SumEntry }_{\min }} \text {; }
$$

The value of Preference $2[i]$ is a combination of $S T D_{i}$ and SumEntry $y_{i}$. The smaller value of $S T D_{i}$ means it may result in a more equal load into each segment. The value 
of SumEntry $y_{i}$ reflects the entries duplication. Hence, maximizing the progress benefit, the value of SumEntry $y_{i}$ should be minimized. The key candidates with the smallest Preference $2[i]$ is selected, so the corresponding partition key is the best one.

Table 6.3 records the partition results when both elementary bit selection and precise bit selection are applied. The maximum number of the entries in all partition buckets declines. Table 6.4 is a comparison result between only implementing elementary bit selection and implementing precise bit selection together. We can see that the power consumption is reduced, while the total entries (entries duplication) slightly increases We conclude that it is worth using precise bit selection.

Table 6.3: Result of Combining the Precise Bit Selection

\begin{tabular}{|l|l|l|l|l|}
\hline Table & $\begin{array}{l}\text { No. of } \\
\text { entries }\end{array}$ & $\begin{array}{l}\text { Sum of Entries } \\
\text { in TCAM }\end{array}$ & $\begin{array}{l}\text { Max Enabled } \\
\text { Entries }\end{array}$ & $\begin{array}{l}\text { Standard } \\
\text { Deviation }\end{array}$ \\
\hline Aads & 63980 & 80768 & 603 & 103.13 \\
Mae-East & 47206 & 61465 & 377 & 88.03 \\
Mae-West & 56032 & 67130 & 417 & 93.26 \\
Paix & 22116 & 25177 & 225 & 44.74 \\
\hline
\end{tabular}

The precise bit selection strategy is different from the one proposed in the previous chapter. The main reason is that before the bit selection step, all the rules have already been divided into a set of buckets in the previous chapter. Therefore, each hash bucket contains a small amount of rules compared with the original table size, for the maximum collision in a hash bucket is usually less than $5 \mathrm{~K}$ and the mean collision in a hash bucket is usually less than $1 \mathrm{~K}$. The rules in one hash bucket may possess kind of "regulation" in 
Table 6.4: Comparisons Between Table 6.3 and Table 6.1

\begin{tabular}{|l|l|l|}
\hline Tables & Increased Entries & $\begin{array}{l}\text { Additional Savings on the Power } \\
\text { Consumption }\end{array}$ \\
\hline Aads & $6.1 \%$ & $16.2 \%$ \\
Mae-East & $3.4 \%$ & $8.7 \%$ \\
Mae-West & $5.6 \%$ & $9.8 \%$ \\
Paix & $4.0 \%$ & $22 \%$ \\
\hline
\end{tabular}

possible bit orders. For example, these three sequences 100001, 010010, 001100 share one pair of hash value $(2,7)$ if $E L=M W=6$. We can see that the " 1 " at the higher position moves to the right while the other " 1 " at the lower position move to the left until the meet. After the elementary bit selection, the situation that bit candidates have the same Preferences often occurs, while it hardly happen in this chapter. Meanwhile, the difference between the Preferences values in the previous chapter is much bigger than the one in this chapter. Hence, the key of the precise selection in the previous chapter is to choose the bits from those having the same Preferences, while the key of the precise selection in this chapter is to choose the bits from the whole bit candidates.

\subsection{Increasing the Table Size TCAMs Can Handle - En- tries Compaction}

In the previous section, $n$ significant bits are chosen to build a $2^{n}$ index table, so 
that the search range narrows down sharply. The number of the bits of each entry that need matching in TCAM is reduced to $32-n$. Furthermore, there is potential ability to increase the possible table size that a TCAM can handle by compacting the forwarding table. Solutions to minimize prefixes in the given forwarding table will be investigated. A two level logic minimization scheme is proposed in [25] to reduce the forwarding table size. The novel entry aggregation method will be described from two aspects.

Firstly, it makes use of the feature of TCAMs. TCAM is a mask based matching device. A basic entry in TCAM is a pair $(p, m)$, where $p$ is the pattern and $m$ is the mask. The variable $m$ indicates the significant bits in $p$ for matching purposes. For example, the basic entry $(1010,1010)$ means that the first and the third bits beginning from the leftmost are significant for matching comparisons and the second and the fourth bits are the "don't care" bits. Therefore, TCAM not only supports the prefix based matching (e.g. (11100, 11110)), but also supports arbitrary mask based matching (e.g. (10100, 10110)). This feature of a TCAM makes it possible for more aggregations, compared to the trie method.

\begin{tabular}{|c|c|c|c|c|}
\hline & Prefix & Mask & Next Hop Port & \multirow{3}{*}{ Original Entries } \\
\hline $\mathrm{P} 1$ & 1001000000 & 1111111100 & 5 & \\
\hline $\mathrm{P} 2$ & 1001100000 & 1111111100 & 5 & \\
\hline & Prefix & Mask & Next Hop Port & \multirow{2}{*}{ Compact Entry } \\
\hline $\mathrm{P} 1 \& \mathrm{P} 2$ & 1001000000 & 1111011100 & 5 & \\
\hline
\end{tabular}

Figure 6.2: Compacting Entries in a Forwarding Table Using Mask-Based Aggregation

Figure 6.2 gives us an example of Mask-based Aggregation. The prefixes, P1 and P2, only differ in the fifth bit. Additionally, both their masks and their next hops are the same. 
Mask-based Aggregation compacts these two entries by setting the fifth bit of the mask to zero. Mask-based Aggregation applies to all the prefix entries in the forwarding table with the same next hop if their prefixes length are the same. This is the Espresso-II algorithm.

\begin{tabular}{|c|c|c|c|c|}
\hline & Prefix & Mask & Next Hop Port & \multirow{4}{*}{ Original Entries } \\
\hline P1 & 1001000000 & 1111100000 & 5 & \\
\hline $\mathrm{P} 2$ & 1001000100 & 1111111100 & 5 & \\
\hline P3 & 1101000000 & 1111111000 & 4 & \\
\hline & Prefix & Mask & Next Hop Port & \multirow{3}{*}{ Compact Entries } \\
\hline $\mathrm{P} 1 \& \mathrm{P} 2$ & 1001000000 & 1111100000 & 5 & \\
\hline P3 & 1101000000 & 1111111000 & 4 & \\
\hline
\end{tabular}

Figure 6.3: Compacting Entries in a Forwarding Table Using Overlapping Aggregation

Secondly, it is resulted from the feature of the forwarding table. There are many overlapping prefixes in a forwarding table. Figure 6.3 gives us an example to compact the overlapping entries in a forwarding table. We call this method Overlapping Aggregation. $\mathrm{P} 2$ is said to be redundant of $\mathrm{P} 1$ and can be aggregated when all of the following conditions are satisfied.

(1) P1 is a parent of P2.

(2) P1 and P2 are sent to the same next hop port.

(3) There is no such prefix P3, which is not only a parent of P2 but also a child of P1, but is sent to a different port from the one $\mathrm{P} 1$ (or $\mathrm{P} 2$ ) is sent to.

In our proposed method, since the forwarding table has already been divided into $2^{n}$ buckets after the first stage, the proposed aggregation algorithm is to compact the entries in each bucket individually, other than in the whole table. Table 6.5 verifies the 
Table 6.5: Results After Entries Comparisons

\begin{tabular}{|l|l|l|l|l|}
\hline Tables & Sum of the Entries & Compaction & Max Entries in a Bucket & Compaction \\
\hline Aads & 65016 & $19.5 \%$ & 445 & $26.2 \%$ \\
Mae-East & 45054 & $26.7 \%$ & 298 & $20.9 \%$ \\
Mae-West & 52093 & $22.4 \%$ & 324 & $22.3 \%$ \\
Paix & 20138 & $20.0 \%$ & 134 & $40.4 \%$ \\
\hline
\end{tabular}

improvement when compaction is applied in each bucket. From this table, either the total entries stored in the TCAM or the maximum collisions the buckets is reduced from $20 \%$ to $40 \%$.

The advantages of compacting entries in each bucket are in two-fold.

Firstly, It makes the compaction much simpler. The compaction operation requires that all the entries have to be considered for entry minimization. The collisions in each bucket is much smaller than the total number of entries in the whole forwarding table. From the results in Table 6.3, we can see that it only needs to examine 600 entries at a time, instead of 60000 entries at a time. In our experiments, the processing time for compaction is reduced by around $20 \%$. It does lead to big improvement in real program, although the theoretical complexity of the compaction is the same.

Secondly, the proposed compacting method makes the updates much simpler. A hundred to a thousand updates per second take place in core routers today approximately[69]. The update operation should be incremental and fast to avoid becoming a bottleneck in the search operation. There are two kinds of updates: partial updates and overall updates. 
The partial updates are the updates confined in a bucket. It is a basic update. Only when the number of the entries in a particular bucket is pretty big, will the overall updates be enabled. The overall updates are to recreate an absolutely new data structure, new significant bits have to be determined. How often we need to recreate the data structure can be decided by real application. In the partial updates, no matter if inserting an entry or deleting an entry, only one bucket is considered. Since the entries are compacted, all the entries share the same bucket have to be taken into account. The related entries are very small. It allows the partial updates to be efficient.

On the other hand, this proposed compacting method is not optimal in that only part of the entries are compared at a time. Does it affect the performance a lot? Comparing with the experiment results in [25], it is also around $20 \%$ compaction, which is at the same improvement level as ours.

\subsection{Range Matching in TCAMs}

TCAM is an efficient device for IP address lookup. But TCAM is not good at performing range matching. A range has to be expanded into prefixes to fit the bit boundary. For example, a popular range $[1024,65535]$ needs to be expanded to, $000001^{*}, 00001^{*}$, $0001^{*}, 001^{*}, 01 *$, and $1 *, 6$ separate prefix entries. In general, the number of expansions could be up to $2 k$, where $k$ is the width of the field [49]. If more than one range field are specified in a rule, the number of expansions is multiplied. For example, if two range fields are applied in a rule and each one is 16-bit wide, there could be up to $32 \times 32=1024$ expansions for a single rule. 


\subsubsection{Range Encoding Scheme (RES)}

The characteristics of the real classifiers are presented in [16]. The following features are what we are interested in:

1. The Transport-layer field is restricted to a small set of values or wildcard '*'. The protocols that a classifier contains mostly are limited in TCP, UDP, ICMP, IGMP, (E)IGRP, GRE, and IPINIP.

2. Only a small portion $(10.2 \%)$ of the specifications for Transport-layer fields are generic range.

3. It is common for many different rules in the same classifier to share a number of field specifications. For example, range filter ' $>1023$ ' is such a common filter in classifiers. From the statistics, it occurs in more than $50 \%$ of the range filters.

Although a classifier could be very large, the number of distinct filters in the Destination/Source Port fields is limited to be very small. In general, these filters are exact values, range fields or wildcards. Therefore, we investigate that if it is possible to encode the filters in those fields by some general mask-based sequences, and store these encoding sequences in the TCAM to replace the original filters. By applying the RES, it is not necessary to expand the range filters any more, which is an efficient way to increase the number of stored rules and reduce the power consumption of a TCAM.

For every incoming packet, we will translate the search keys into encoded sequences before matching against TCAM. The translation could be completed in a single memory access by direct table lookup. 
The benefits from the RES-based TCAM packet classification algorithm are in the following aspects:

1. We try to minimize the width of the encoding sequence. Sometimes the width of the encoding sequences could be longer than the width of the range filter itself, when the number of distinct filters is very big. We are going to discuss how to restrict the width expansion of RES.

2. It requires less TCAM storage space. Our scheme can handle large classifiers in a single TCAM to reduce the system cost and power consumption.

3. The search time of the worst case is guaranteed. It is not like those search tree based algorithms that the search time varies a lot depending on the where the search key locates.

Table 6.6: An Example of Range Set

\begin{tabular}{|l|l||l|l|}
\hline ID. & Ranges & ID. & Ranges \\
\hline A & $20-40$ & D & $25-32$ \\
B & $10-15$ & E & 14 \\
C & 7 & F & $35-45$ \\
\hline
\end{tabular}

Table 6.6 is an example of a range filters set. In this set, there are 6 distinct filters, 2 of which are exact values and 4 of which are ranges. They can be represented on a line as in Figure 6.4 (A). Some of the ranges are overlapped. Then we will explain how to encode these filters in Table 6.6. 


\section{Direct Encode}

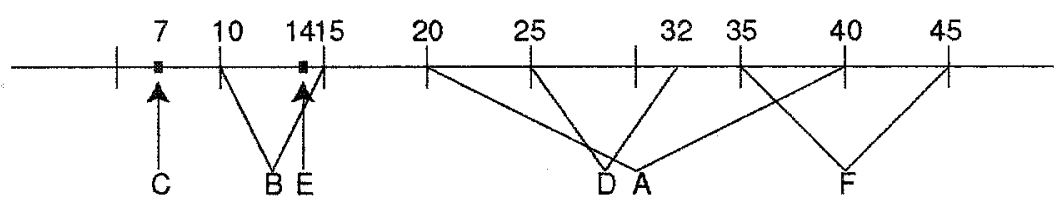

(A)

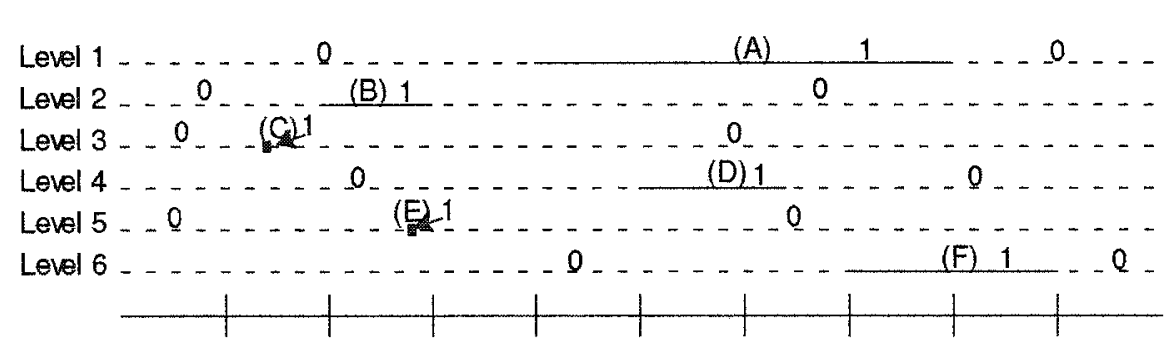

\begin{tabular}{|c|c|c|c|c|c|}
\hline $\mathrm{A}$ & $\mathrm{B}$ & $\mathrm{C}$ & $\mathrm{D}$ & $\mathrm{E}$ & $\mathrm{F}$ \\
\hline 1 & $\mathrm{X}$ & $\mathrm{X}$ & $\mathrm{X}$ & $\mathrm{X}$ & $\mathrm{X}$ \\
\hline $\mathrm{X}$ & 1 & $\mathrm{X}$ & $\mathrm{X}$ & $\mathrm{X}$ & $\mathrm{X}$ \\
\hline $\mathrm{X}$ & $\mathrm{X}$ & 1 & $\mathrm{X}$ & $\mathrm{X}$ & $\mathrm{X}$ \\
\hline $\mathrm{X}$ & $\mathrm{X}$ & $\mathrm{X}$ & 1 & $\mathrm{X}$ & $\mathrm{X}$ \\
\hline $\mathrm{X}$ & $\mathrm{X}$ & $\mathrm{X}$ & $\mathrm{X}$ & 1 & $\mathrm{X}$ \\
\hline $\mathrm{X}$ & $\mathrm{X}$ & $\mathrm{X}$ & $\mathrm{X}$ & $\mathrm{X}$ & 1 \\
\hline
\end{tabular}

(B)

Level $1 \ldots .00$ (C) 101 (B) $10: 00$ (A) 11 $00 \ldots$ Level $2 \ldots 00 \ldots$ (E) . . . . . . . . 00 (D) $10.00 \quad$ (F) $11-00$

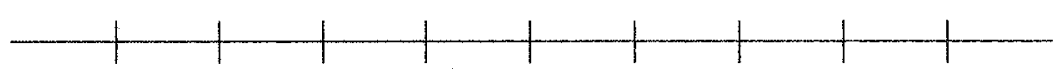

\begin{tabular}{|l|l|l|l|l|l|}
\hline $\mathrm{A}$ & $\mathrm{B}$ & $\mathrm{C}$ & $\mathrm{D}$ & $\mathrm{E}$ & $\mathrm{F}$ \\
\hline 1 & 1 & 0 & $\mathrm{X}$ & $\mathrm{X}$ & $\mathrm{X}$ \\
1 & 0 & 1 & $\mathrm{X}$ & $\mathrm{X}$ & $\mathrm{X}$ \\
\hline $\mathrm{X}$ & $\mathrm{X}$ & $\mathrm{X}$ & 1 & 0 & 1 \\
$\mathrm{X}$ & $\mathrm{X}$ & $\mathrm{X}$ & 0 & 1 & 1 \\
\hline
\end{tabular}

(C)

Figure 6.4: Re-Encode Filters According to Different Levels

Suppose that there are $n$ distinct filters in a range filters set, $\mathcal{F}=\left\{F_{1}, F_{2}, \ldots, F_{n}\right\}$. We build additional $n$ one-dimensional spaces. Let $F_{i}$ be located in the corresponding $i^{\text {th }}$ level space. At any level $i$, the interval that $F_{i}$ covers is assigned 1 , and the remaining intervals are assigned $0 \mathrm{~s}$, as shown in Figure 6.4 (B). We can use an $n$ bit binary sequence, $\mathcal{B}=B_{1} B_{2} \ldots B_{n}$, to represent each filter. Let $\mathcal{B}$ for filter $F_{i}$ be expressed as the following rules: the $i^{\text {th }}$ bit of $\mathcal{B}$ is set to 1 , i.e. $B_{i}=1$, and all the other bits are set to ' $x^{\prime}\left(\right.$ don't $^{\prime}$ care $)$. In the given example in Table 6.6, all the filters are encoded to six 6-bit long sequences. 
Filter $A$ is encoded to $1 \times \times \times \times \times$, and filter $F$ is encoded to $\times \times \times \times \times 1$. After RES, those $n$ range-based filters are represented by a set of $n$-bit wide general mask-based filters, which can be stored in TCAM easily.

Unfortunately, when the number of distinct range filters, $n$, is big, the encoding sequences are very long. Then we will discuss how to reuse space efficiently to reduce the bits of encoding sequences.

\section{Improvement}

A single bit is used to represent each distinct range. Since some ranges are totally independent, or no overlap at all, it is possible to allow them to be located in the same level space. All $n$ distinct filters are partitioned into $k$ level spaces, where $k \leq n$. Suppose that the ranges $\mathcal{F}_{i}=\left\{F_{i_{1}}, F_{i_{2}}, \ldots, F_{i_{L_{i}}}\right\}$ are assigned to level $i(i \leq k)$. The filters in $\mathcal{F}_{i}$ must satisfy the following conditions:

(1) For $\forall j, F_{i_{j}} \in \mathcal{F}$, where $j \leq L$.

(2) For $\forall j,(j \neq m), \exists F_{i_{j}} \cap F_{i_{m}}=\emptyset$, where $F_{i_{j}}, F_{i_{m}} \in \mathcal{F}_{i}$.

At any level $i, M_{i}=\left\lceil\log _{2}\left(L_{i}+1\right)\right\rceil$ bit binary sequences can be used to encode the filters in set $\mathcal{F}_{i}$. Let $\left.\operatorname{Bit}(a)\right|_{M_{i}}$ denote an $M_{i}$ bit binary sequence, which is equal to the value of integer $a$. For instance, $\left.B i t(4)\right|_{4}$ expresses the binary sequence 0100 . Bit $\left.(x)\right|_{4}$ expresses the binary sequence $\times \times \times \times$. At level $i$, all the intervals that are not covered by any filter in $\mathcal{F}_{i}$ are expressed as $\left.\operatorname{Bit}(0)\right|_{M_{i}}$. All the other intervals that are covered by any filter in $\mathcal{F}_{i}$ are expressed from $\left.\operatorname{Bit}(1)\right|_{M_{i}}$ to $\left.\operatorname{Bit}(L)\right|_{M_{i}}$. Totally, a $\left(M_{1}+M_{2}+\cdots+M_{k}\right)$ bit long sequence, $\mathcal{B}=\left.\left.\left.\operatorname{Bit}(\cdot)\right|_{M_{1}} B i t(\cdot)\right|_{M_{2}} \ldots B i t(\cdot)\right|_{M_{k}}$, can represent a filter. Assume that $F_{j} \in \mathcal{F}_{i}$ and $F_{j}=F_{i_{m}} . F_{j}$ can be expressed as such a binary sequence that only section 
$\left.\operatorname{Bit}(\cdot)\right|_{M_{i}}$ is set to $\left.\operatorname{Bit}(m)\right|_{M_{i}}$ and all the other sections are set to $\left.\operatorname{Bit}(\times)\right|_{M_{l}}$, where $l \neq i$.

Figure $6.4(\mathrm{C})$ is the example of partitioning 6 filters in Table 6.6 into 2 level spaces: 3 filters in each level space. A 2 bit sequence is applied in each level. For example, filter $A$ is encoded to $11 \times \times$ and filter $F$ is encoded to $\times \times 11$, which are 2 bit shorter than the ones in (B).

\section{Search Key Encoding}

We will explicate how to encode the search key in range fields, before it matches against TCAM.

A search key $S \in\left[0,2^{L}-1\right]$ is translated into a binary string, $B_{1} B_{2} \ldots B_{k}$, using improved RES, assuming all ranges are partitioned into $k$ level spaces. For any $i(1 \leq i \leq k)$, if there exist an $l\left(1 \leq l \leq L_{i}\right)$, such that $S \in F_{i_{l}}$, then $B_{i}=\left.B i t(l)\right|_{M_{i}}$, otherwise, $B_{i}=\left.B i t(0)\right|_{M_{i}}$. For instance, the search key 14 is encoded to 1001 with respect to the example in Table 6.6 and the encoding rule in Figure 6.4 (C), for it matches both rule B and rule E. A complete direct translate lookup table is shown in Figure 6.5. Search key encoding can be implemented as a one step direct table lookup. For a 16-bit wide range field, it needs an encoding table with $2^{16}$ entries.

\section{Performance of RES}

The rule with an exact value is a special range. Suppose that there are $p$ rules with exact values in all $n$ distinct rules. All the $p$ rules with exact values are mutually independent and can be partitioned into the same level space. In the worst case, all the left $(n-p)$ range rules are all overlapped with each other, then all of the range rules 


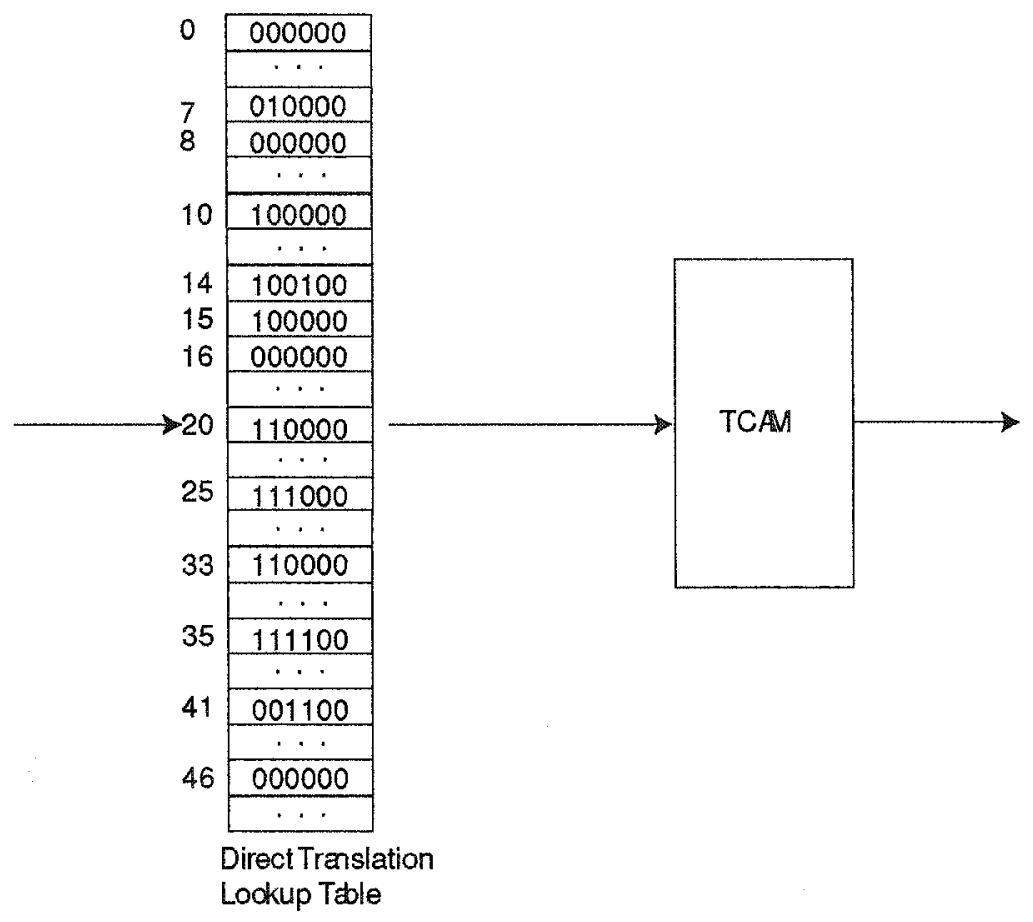

Figure 6.5: Extended TCAM-Based Packet Classification

are partitioned into distinct spaces. The upper bound width of the encoding sequences is $\left\lceil\log _{2}(p+1)\right\rceil+(n-p)$.

In real simulation, we partition the range rules into two flows, in which, flow one is for the rules with exact values and flow two is for the rules with ranges. All the rules in flow one are partitioned into one space. Then, we examine all the rules in flow two so that a set of independent rules is located in one level space. We assume that the width of a range field is 16 bit. We randomly generate $50,100,200$, and 300 distinct range rules. Table 6.7 tells us the width of the encoding sequences when improved RES is applied. We believe that the number of distinct ranges in real life classifiers is no more than 300 [16]. 
Table 6.7: Width of Encoding Sequences

\begin{tabular}{|l|l|l|l|l|}
\hline No. of distinct Rules & 50 & 100 & 200 & 300 \\
\hline Width of Encoded vector (Bit) & 10 & 19 & 25 & 35 \\
\hline
\end{tabular}

\subsubsection{RES + TCAM in Packet Classification}

Some multi-dimensional classifiers contain range fields (Destination Port field or Source Port field). Suppose that these multi-dimensional classifiers are not so big, e.g. less than $128 \mathrm{~K}$ entries in IPv4. All the filters in the range fields are translated into general mask based encoding sequences. The encoding sequences replace the filters in those range fields to be stored in the TCAM.

All the rules are partitioned into several buckets with respect to the values of the bits on some significant positions in the prefix based fields, as in the previous section. During each lookup procedure, only one of the bucket is enabled for the matching process in TCAM.

In the real classifications, first, the values in the range fields in the incoming header are translated into encoding sequences by looking into RES direct table for each range field. Then a one step optimal entries partition is processed in the next. Only one segment of the TCAM cells are triggered for parallel matching. It is an efficient approach that not only reduces the power consumption but also restricts the entries expansion. 


\subsection{Summary}

In this chapter, we proposed some techniques to allow TCAMs more efficient for today's IP address lookup and packet classification algorithms.

Firstly, we proposed an advanced bit selection method. The purpose of this method is to lowering down the power consumption. The proposed approach a two stage search procedure. An optimal index table is built based on $n$ most significant bits in front of TCAMs, and each entry in it points to one certain segment of the total entries. Only one segment of all the entries is enabled for comparison. Therefore, the search range is reduced to be one out of $O\left(2^{n}\right)$ sharply for match processing in TCAMs .

Secondly, a table compaction method was presented to aggregate the rules in each segment in TCAMs. A pair of forwarding table compaction methods, Mask-based Aggregation and Overlapping Aggregation, are applied in each segment individually. The experimental results provide sufficient evidence that this architecture design performs well for real tables and is convenient for update. It is also scalable to IPv6.

Thirdly, we concentrated on solving range matching problems in TCAMs. By examining the classifier, we find that the number of distinct range filters is pretty small compared to the number of rules in a classifier. We proposed a RES method to encode range filters to general mask based binary sequences and those encoding sequences are stored in the TCAMs. It can avoid entry duplication. 


\section{Chapter 7}

\section{Conclusions and Future Work}

In the previous chapters, we detailed our contributions to the thesis. In this chapter, we summarize these contributions. At the same time, we discuss the future work we would like to continue.

\subsection{Conclusions}

Three high performance table lookup algorithms are presented in this thesis. The three algorithms are an IP address lookup algorithm, an packet classification algorithm and the application of TCAM in table lookup algorithms. Their goals are to reduce memory requirement, to increase parallel implementation possibility and to scale to IPv6. 


\subsubsection{Contributions of the IP Address Lookup Based on Comb Ex- traction Scheme}

In Chapter 4, we proposed an IP address lookup algorithm based on CES. Its main contribution is to transform a single sequential address lookup procedure to a pair of parallel small sub-lookup procedures, in order to reduce the processing time. The proposed CES partition approach is able to allow the pair of sub-lookups balanced. It maximizes the benefit from parallel processing.

In the proposed algorithm, it is necessary to compare each possible pair of matching prefixes from sub-lookups. We can take advantage of today's technology not only to parallelize sub-lookups and comparisons between the matching sub-prefixes, but also to minimize the comparison time.

Finally, we also proposed distinct system architectures and their corresponding search strategies. The experimental results show that both architectures are able to provide outstanding average searching performance and their storage requirements are small.

\subsubsection{Contributions of the Packet Classification Using Bit Character- istics}

In Chapter 5, we proposed a packet classification algorithm using bit characteristics. Its main contribution is to allow the bits from all the dimensions useful at each step. This algorithm contains three layers: hash table lookup, optimal search trie lookup and simple search in small sets. 
The hash function, (Sum1, Sum2), is built to represent the 1s or 0s distribution of the bits extracted from all the dimensions. This function takes advantage of this feature to reduce the collisions in hash buckets. It also can reduce the effect of wildcards. The hash table is small enough to be stored in fast memory.

An optimal search trie is built in each hash bucket. A node in a search trie becomes a final leaf until the number of the rules fall into it is no more than a pre-defined size. The most significant bits are selected as a search key with respect to the bit distribtion. Each search trie may be a multi-way trie.

In the final small set, the number of the rules is pretty small. It is possible to use a linear search or parallel search to find the final matching rule.

The experimental results show that the proposed algorithm has excellent performance for multi-dimensional table lookup problems. It has reasonable storage cost and fast search speed. It is especially suitable for big classifiers. The proposed algorithm is also scalable to IPv6.

\subsubsection{Contributions of the TCAMs in Table Lookup Algorithms}

In Chapter 6, we proposed some approaches to make the TCAMs more efficient in table lookup algorithms. Its main contribution is to solve power consumption, limited storage capability and range matching problems in real applications.

Firstly, we proposed an approach to partition all the entries into several segments based on some significant bits. Therefore only one segment of the entries in the TCAM is enabled for matching after a direct table lookup. This is an effective way to reduce the power consumption. There is a trade-off between entry duplication and power consump- 
tion, when choosing the number of significant bits.

Secondly, we proposed an approach to minimize the number of the entries in a table. The minimization consists of overlapping aggregation and mask-based aggregation. The aggregation procedure is operated in each segment, therefore, both the original compacting and the update become simpler. This is an effective way to increase the table size that a TCAM can handle.

Finally, we proposed an approach to solve range matching problems. The ranges are encoded to mask-based sequences by RES. It is an efficient method to avoid the entry duplication for range matching in TCAMs.

The experimental results show that these three approaches can improve the performance of TCAM in real applications. Since TCAM has its own memory limitation and high cost, it is only useful for the tables that are not very big. These approaches are also scalable to IPv6.

\subsection{Future Work}

All the existing IP address lookup and packet classification algorithms are dedicated to Internet forwarding in IPv4. IPv6, however, will replace IPv4 due to the growing shortage of available IP addresses and upcoming deployment of $3 \mathrm{G}$ or more advanced mobile networks. The changes significantly degrade the performance of the existing fast IP lookup approaches, especially in the design of high-speed switch/router with NPU. Although the proposed algorithms in the thesis are scalable to IPv6, we can hardly get real-life table data in IPv6 right now. In the future work, we will try to adapt the proposed 
algorithms in IPv6 more efficiently.

Traditional queuing and differentiation methods rely on gross categories such as source or destination address, protocols, fixed application port numbers, or initiating ports as classification fields. However, the current network traffic has changed considerably. Customers are requesting service level agreements that specify not only traffic from a specific address, but also that traffic for specific applications is delivered with priority. To meet these demands, networks are becoming aware not only of the data streams but also of the content of those streams and how that content must be serviced. This is an advanced hot topic in the future. 


\section{Bibliography}

[1] J. Alvarez, B. Hajek, On the use of packet classes in communication networks to enhance congestion pricing based on marks, Automatic Control, IEEE Transactions on , 47(6), June 2002, $1020-1026$.

[2] B.A. Al-Khaffaf, E.K. Karuppiah and R. Abdulah, Efficient partition based IPv6 lookup algorithm for packet forwarding, Communications, 2003. APCC 2003. The 9th Asia-Pacific Conference on, Sept. 2003, 238-242.

[3] M. J. Akhbarizadeh and M. Nourani, An IP Packet Forwarding Technique Based on Partitioned Lookup Table, ICC'02, April, 2002.

[4] F. Baboescu, G. Varghese, Scalable Packet Classification, Proceedings of ACM SIGCOMM, Aug. 2001.

[5] R. Balapumi, E.K. Karuppiah and R. Abdullah, IPv6 anycast address lookup using trier-based algorithm Communications, 2003. APCC 2003. The 9th AsiaPacific Conference on, Sept. 2003, 1082-1086. 
[6] M. M. Buddhikot, S. Suri, and M. Waldvogel, Space Decomposition Techniques for Fast Layer-4 Switching, Proceedings of Conf. Protocols for High Speed Networks, Aug. 1999,25-41.

[7] H. J. Chao,Next Generation Routers,Proceedings of the IEEE, Sept. 2002, 1518 1558 .

[8] L. Choi, S.W. Kim, and J. Joung, Packet classification through hierarchical rulebase partitioning, Electronics Letters, 4(18), Sept. 2004, 1158 - 1159.

[9] Yie-Tarng Chen, and Shin-Shian Lee, An efficient packet classification algorithm for network processors, Communications, 2003. ICC '03. IEEE International Conference on , May 2003, $1596-1600$.

[10] W.T. Chen, S.B. Shih, and J.L. Chiang, A two-stage packet classification algorithm, Advanced Information Networking and Applications, 2003. AINA 2003. 17th International Conference on, March 2003, 762 - 767.

[11] J.-H. Cherng, H.W. Wu, W.H. Lin, and W.S.E. Chen, Fast diagonal packet classification on tuple space - dealing with conflict free filters, Personal, Indoor and Mobile Radio Communications, 2003. PIMRC 2003. 14th IEEE Proceedings on , Sept. 2003, $89-93$.

[12] H. Che, Y. Wang and Z. Wang, A rule grouping technique for weight-based TCAM coprocessors [packet classification application], High Performance Interconnects, 2003. Proceedings. 11th Symposium on, Aug. 2003, 32-37. 
[13] M. Degermark, A. Brodnik, S. Carlsson, and S. Pink, Small forwarding tables for fast routing lookups, Proceedings of ACM SIGCOMM, Sept. 1997, 3-14.

[14] P. Gupta, Algorithms for Routing Lookups and Packet Classificaiton, doctoral dissertation, Dept. Computer Science, Stanford Univ., 2002.

[15] D. Ginsburg and M. Hattar, Implementing IP Services at the Network Edge, Pearson Education, Inc. 2002.

[16] P. Gupta and N. McKeown, Packet Classification on Multiple Fields, Proceedings of ACM SIGCOMM, Sept. 1999, 147-160.

[17] P. Gupta and N. McKeown, Classification Using Hierarchical Intelligent Cuttings, Proceedings of Hot Interconnects VII, Aug. 1999.

[18] P. Gupta and N. McKeown, Algorithms for packet classification, Network, IEEE , 15(2), March-April 2001,24 - 32.

[19] S. Iyer, R. Rao Kompella, and A. Shelat, ClassiPl: an architecture for fast and flexible packet classification, Network, IEEE, 15(2), March-April 2001,33 - 41'

[20] E.K. Karuppiah and R. Abdullah, Global IPv6 anycast address lookup with $n p$ Communications, 2003. APCC 2003. The 9th Asia-Pacific Conference on, Sept. $2003,1106-1110$.

[21] V.P. Kumar, T.V. Lakshman, and D. Stiliadis, Beyond Best Effort: Router Architectures for the Differentiated Services of Tomorrow's Internet, IEEE Communications Magazine, May 1998, 152-164. 
[22] M. Kobayashi, T. Murase, and A. Kuriyama, A Longest Prefix Match Search Engine for Multigigabit IP Processing, Proc. IEEE Int'1 Conf. Comm. 2000, 13601364.

[23] S. Kaxiras, G. Keramidas, IPStash: a power-efficient memory architecture for IP-lookup, Microarchitecture, 2003. MICRO-36. Proceedings. 36th Annual IEEE/ACM International Symposium on, Dec. 2003, 361 - 372.

[24] Chung Keung Poon, Andy Kwok, Space optimal packet classification for 2D conflict-free filters, Parallel Architectures, Algorithms and Networks, 2004. Proceedings. 7th International Symposium on, May 2004, 260 - 265.

[25] H. Liu, Routing Table Compaction in Ternary CAM, IEEE Micro. 22(1), Jan.Feb. 2002, 58-64.

[26] H. Liu, Efficient mapping of range classifier into ternary-CAM, High Performance Interconnects, 2002. Proceedings. 10th Symposium on, Aug. 2002, 95 100

[27] Po-Chou Lin and Chung-Ju Chang, A priority TCAM IP-routing lookup scheme, Communications Letters, IEEE ,7(7), July 2003, 337-339.

[28] K. Li, F. Chang, D. Berger, and W. Feng, Architectures for packet classification caching, Networks, 2003. the 11th IEEE International Conference on, Sept. 2003, $111-117$.

[29] H. Lim and Y. Jung, A parallel multiple hashing architecture for IP address lookup, High Performance Switching and Routing, 2004, 91 - 95. 
[30] C. Labovitz, G.R. Malan, and F. Jahanian, Internet Routing Instability, IEEE/ACM Trans. on Networking, 6(5), Oct. 1998, 515-528.

[31] T. V. Lakshman and D. Stidialis, High Speed Policy-based Packet Forwarding Using Efficient Multi-dimensional Range Matching, Proc. SIGCOMM'98, Sept. 1998 .

[32] B. Lampson, V. Srinivasan and G. Varghese, IP lookups Using Multiway and Multicolumn Search, IEEE ACM Transactions on Networking, 7(3), June 1999, 324-334.

[33] Zhiyong Liang, Jianping $\mathrm{Wu}$ and $\mathrm{Ke} \mathrm{Xu}, A$ TCAM-based IP lookup scheme for multi-nexthop routing ICCNMC 2003, 128 - 135.

[34] D. R. Morrison, PATRICIA - Practical Algorithm to Retrieve Information Coded in Alphanumeric, Journal of the ACM, 15(4), Oct. 1968, 514-534.

[35] H. Miyoshi, J.S. Thompson, SIRS: selective intersected rule search for packet classification, Networks, 2003. ICON2003. The 11th IEEE International Conference on, Sept. 2003, $105-110$.

[36] M.M. Moallem, N. Yazdani, K. Faez, H. Taheri, A multilayer neural network for IP lookup and packet classification, Communications, 2003. APCC 2003. The 9th Asia-Pacific Conference on, Sept. 2003, 924 - 928.

[37] S. Nilsson and G. Karlsson, IP-Address Lookup Using LC-Tries, IEEE Journal on Selected Areas in Communications, 17(6), June 1999, 1083-1092. 
[38] Rita Pužmanová, Routing And Switching - Time of Convergence?, Pearson Education, Inc. 2002.

[39] D. Pao, C. Liu, A. Wu, L. Yeung, and K.S. Chan, Efficient hardware architecture for fast IP address lookup, Computers and Digital Techniques, IEE Proceedings, 150(1), 20 Jan. 2003, $43-52$.

[40] A. Prakash, R. Kotla, T. Mandal, and A. Aziz, A high-performance architecture and BDD-based synthesis methodology for packet classification, ComputerAided Design of Integrated Circuits and Systems, IEEE Transactions on , 22(6), June 2003, $698-709$.

[41] R. Panigrahy and S. Sharma, TCAM Power Consumption and Increasing Throughput, Proc. 10th Symp. High-Performance Interconnects 2002, 107-112.

[42] M.A. Ruiz-Sanchez, E.W. Biersack, and W. Dabbous,Survey and toxonomy of IP address lookup algorithms, Network, IEEE ,15(2), March-April 2001, 8 - 23.

[43] V.C. Ravikumar and R.N. Mahapatra, TCAM architecture for IP lookup using prefix properties, Micro, IEEE, 24(2), March-April 2004, 60-69.

[44] L. Qiu, G. Varghese, and S. Suri, Fast Firewall Implementations for Software and Hardware-based Routers, Proc. 9th International Conference on Network Protocols (ICNP'2001), November 2001.

[45] K. Sklower, A Tree-Based Packet Routing Table for Berkely Unix, Proc. 1991 Winter Usenix Conf., 1991, 93-99. 
[46] Ching-Fong Su, High-speed packet classification using segment tree, GLOBECOM 2000, Nov. 2000, 582 - 586.

[47] Xuehong Sun, IP Addess Lookup and Packet Classification Algorithms, doctoral dissertation, School of Mathematics and Statistics, Carleton University, 2003.

[48] D. Shah and P. Gupta, Fast Updating Algorithms for TCAMs, IEEE Micro. 21(1), Jan-Feb. 2001, 36-47.

[49] V. Srinivasan, S. Suri, and G. Varghese, Packet Classification Using Tuples Space Search, Proceedings of ACM SIGCOMM, 1999.

[50] E. Spitznagel, D. Taylor and J. Turner, Packet classification using extended TCAMs, Network Protocols, 2003. Proceedings. 11th IEEE International Conference on, Nov. 2003, 120-131.

[51] V. Srinivasan and G. Varghese, Fast address lookups using controlled prefix expansion, Transactions on Computer Systems, 17(1), Feb. 1999, 1-40.

[52] V. Srinivasan, G. Varghese, S. Suri, and M. Waldvogel, Fast and Scalable Layer Four Switching, Proceedings of ACM SIGCOMM, Sept. 1998, 203-214.

[53] Liqin Tian, Chuang Lin, and Zhangxi Tan, A fast packet classification algorithm based on classifier's characteristic applying to multi-fields, Communication Technology Proceedings, 2003. ICCT 2003. International Conference on, April 2003, 255 - 258.

[54] Administering Cisco Q0S for IP Networks, Syngr ess Publishing, Inc., 2001. 
[55] Yiyan Tang, Lie Qian, B. Bou-Diab, A. Krishnamurthy, G. Damm, and Yuke Wang, High-performance implementation for graph-based packet classification algorithm on network processor, Communications, 2004 IEEE International Conference on , June 2004, 1268 - 1272.

[56] M. Uga and K. Shiomoto, A novel ultra high-speed multi-layer table lookup method using TCAM for differentiated services in the Internet, High Performance Switching and Routing, 2001 IEEE Workshop on, May 2001, 240-244.

[57] J. van Lunteren, A.P.J. Engbersen, Multi-field packet classification using ternary CAM, Electronics Letters ,38(1), Jan. 2002, 21 - 23.

[58] J. Wang and K. Nahrstedt, Parallel IP Packet Forwarding for Tomorrow's IP Routers, IEEE Workshops on High Performance Switching and Routing, May 2001, 353-357.

[59] T. Woo, A modular approach to packet classification: Algorithms and results, Proceedings of INFOCOM, 2000.

[60] M. Waldvogel, G. Varghese, J. Turner, and B. Plattner, Scalable High Speed IP Routing Lookups, Proceedings of ACM SIGCOMM, Sept. 1997, 25-36.

[61] Zhen-Xing Wang, He-Ming Wang, Ya-Min Sun, Yan-Xiao Zhang and Jiang-Xing Wu, High-performance IPv4/IPv6 dual-stack routing lookup, Advanced Information Networking and Applications, 2004. AINA 2004. 18th International Conference on, March 2004, 476-481. 
[62] Yuke Wang, Yun Zhang, Yiyan Tang, A. Krishnamurthy, G. Damm, and B. BouDiab, Novel disjoint graph based algorithm for multi-field range-based packet classification, Communications, 2004 IEEE International Conference on, June $2004,1108-1112$.

[63] J. Xu, M. Singhal, and J. Degroat, A novel cache architecture to support layerfour packet classification at memory access speeds, INFOCOM 2000. Nineteenth Annual Joint Conference of the IEEE Computer and Communications Societies. Proceedings. IEEE, March 2000, 1445 - 1454.

[64] Zhen Xu, G. Damm, I. Lambadaris, and Y.Q. Zhao, IP packet forwarding based on comb extraction scheme, Communications, 2004 IEEE International Conference on, June 2004, 1065 - 1069.

[65] Zhen Xu, I. Lambadaris, and Y.Q. Zhao, Packet Classification Using Bit Characteristics, Accepted by IEEE CCECE05.

[66] Zhen Xu, I. Lambadaris, and Y.Q. Zhao, IP Address Lookup based on Efficient TCAMs, Accepted by IEEE CCECE05.

[67] Ruoyuan Zhang, A Scalable Algorithm for Packet Classification, Master thesis, Department of Systems and Computer Engineering, Carleton University, 2003.

[68] G. Zhang, H.J. Chao, J. Joung, Fast packet classification using field-level trie, GLOBECOM 2003, Dec. 2003, 3201 - 3205.

[69] F. Zane, G. Narlikar, and A. Basu, CoolCAMs: Power-Efficient TCAMs for Forwarding Engines, Proc. IEEE Infocom 2003, 42-52. 
[70] BGP Routing Table, http://bgp.potaroo.net.

[71] Intel Network Processors, http://www.intel.com/design/network/products/npfamily.

[72] M. Kohler, Introduction to Network Processors, http://www.netrino.com/Articles/NetworkProcessors.

[73] Xilinx CAMs, http://www.xilinx.com/esp/networks_telecom/optical/xlnx_net/cam.htm.

[74] SiberCam Ultra-18M, http://www.sibercore.com/pdf/SCT1842_ProdBrief.pdf.

[75] CIDR-Q\&A, http://www.geocities.com/SiliconValley/Vista/8672/network/cidr.html.

[76] V.P. Kumar, T.V. Lakshman and D. Stiliadis, Beyond Best Effort: Router Architectures for the Differentiated Services of Tomorrow's Internet, http://www.belllabs.com/user/stiliadi/router/router.html.

[77] EZchip Technologies, http://www.ezchip.com.

[78] HyWire Ltd., http://www.hywire.com.

[79] Linley Group, http://www.linleygroup.com.

[80] Network Processing Forum, http://www.npforum.org.

[81] SiberCore Technologies, http://www.sibercore.com.

[82] Integrated Device Technology, Inc., http://www.idt.com.

[83] NetLogic Microsystems, Inc., http://www.netlogicmicro.com.

[84] Cypress Semiconductor Corporation, http://www.cypress.com. 
[85] Internet Protocol, http://www.networksorcery.com/enp/protocol/ip.htm.

[86] IPv6 Information (1), http://www.ipv6.org.

[87] IPv6 Information (2), http://playground.sun.com/pub/ipng/html/ipng-main.html. 